\title{
\begin{tabular}{l|l} 
Mibraries & DSpace@MIT
\end{tabular}
}

\author{
MIT Open Access Articles
}

\section{A TIGHT CONNECTION BETWEEN GAMMA-RAY OUTBURSTS AND PARSEC-SCALE JET ACTIVITY IN THE QUASAR 3C 454.3}

The MIT Faculty has made this article openly available. Please share how this access benefits you. Your story matters.

Citation: Jorstad, Svetlana G., Alan P. Marscher, Paul S. Smith, Valeri M. Larionov, Iván Agudo, Mark Gurwell, Ann E. Wehrle, et al. “A TIGHT CONNECTION BETWEEN GAMMA-RAY OUTBURSTS AND PARSEC-SCALE JET ACTIVITY IN THE QUASAR 3C 454.3.” The Astrophysical Journal 773, no. 2 (August 5, 2013): 147. (C) 2013 American Astronomical Society.

As Published: http://dx.doi.org/10.1088/0004-637x/773/2/147

Publisher: Institute of Physics/American Astronomical Society

Persistent URL: http://hdl.handle.net/1721.1/95699

Version: Final published version: final published article, as it appeared in a journal, conference proceedings, or other formally published context

Terms of Use: Article is made available in accordance with the publisher's policy and may be subject to US copyright law. Please refer to the publisher's site for terms of use. 


\title{
A TIGHT CONNECTION BETWEEN GAMMA-RAY OUTBURSTS AND PARSEC-SCALE JET ACTIVITY IN THE QUASAR 3C 454.3
}

\author{
Svetlana G. Jorstad ${ }^{1,2}$, Alan P. Marscher ${ }^{1}$, Paul S. Smith ${ }^{3}$, Valeri M. Larionov ${ }^{2,4,5}$, Iván Agudo ${ }^{1,6}$, Mark Gurwell ${ }^{7}$,

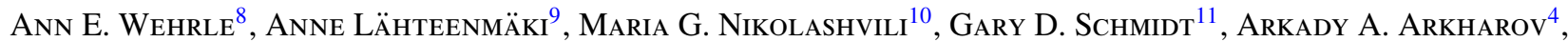 \\ Dmitry A. Blinov ${ }^{2}$, Kelly Blumenthal ${ }^{1}$, Carolina Casadio ${ }^{6}$, Revaz A. Chigladze ${ }^{10}$, Natalia V. Efimova ${ }^{2,4}$, \\ Joseph R. EgGen ${ }^{12}$, José L. Gómez ${ }^{6}$, Dirk Grupe ${ }^{13}$, Vladimir A. Hagen-Thorn ${ }^{2,5}$, Manasvita Joshi ${ }^{1}$, Givi N. Kimeridze ${ }^{10}$, \\ Tatiana S. Konstantinova ${ }^{2}$, Evgenia N. Kopatskaya ${ }^{2}$, Omar M. Kurtanidze ${ }^{10}$, Sofia O. Kurtanidze ${ }^{10}$, \\ Elena G. Larionova ${ }^{2}$, Liudmilla V. Larionova ${ }^{2}$, Lorand A. Sigua ${ }^{10}$, Nicholas R. MacDonald ${ }^{1}$, Jeremy D. Maune ${ }^{12}$, \\ Ian M. McHardy ${ }^{14}$, H. Richard Miller ${ }^{12}$, Sol N. Molina ${ }^{6}$, Daria A. Morozova ${ }^{2}$, Terri Scott ${ }^{1}$, Brian W. Taylor ${ }^{1,15}$, \\ Merja Tornikoski ${ }^{9}$, Ivan S. Troitsky ${ }^{2}$, Clemens Thum ${ }^{16}$, Gary Walker $^{17}$, Karen E. Williamson ${ }^{1}$, \\ Stephanie Sallum ${ }^{17,18}$, Santina Consiglio $^{17,19}$, and Vladimir Strelnitski ${ }^{17}$ \\ ${ }^{1}$ Institute for Astrophysical Research, Boston University, 725 Commonwealth Avenue, Boston, MA 02215, USA; jorstad@ bu.edu \\ ${ }^{2}$ Astronomical Institute, St. Petersburg State University, Universitetskij Pr. 28, Petrodvorets, 198504 St. Petersburg, Russia \\ ${ }^{3}$ Steward Observatory, University of Arizona, Tucson, AZ 85721-0065, USA \\ ${ }^{4}$ Main (Pulkovo) Astronomical Observatory of RAS, Pulkovskoye shosse, 60, 196140 St. Petersburg, Russia \\ ${ }^{5}$ Isaac Newton Institute of Chile, St. Petersburg Branch, St. Petersburg, Russia \\ ${ }^{6}$ Instituto de Astrofísica de Andalucía, CSIC, Apartado 3004, E-18080 Granada, Spain \\ ${ }^{7}$ Harvard-Smithsonian Center for Astrophysics, 60 Garden Street, Cambridge, MA 02138, USA \\ ${ }^{8}$ Space Science Institute, 4750 Walnut Street, Boulder, CO 80301, USA \\ ${ }^{9}$ Aalto University Metsähovi Radio Observatory Metsähovintie 114, FIN-02540 Kylmälä, Finland \\ ${ }_{10}$ Abastumani Astrophysical Observatory, Mt. Kanobili, Abastumani, Georgia \\ ${ }^{11}$ National Science Foundation, 4201 Wilson Avenue, Arlington, VA, 22230, USA \\ ${ }^{12}$ Department of Physics and Astronomy, Georgia State University, Atlanta, GA 30303-3083, USA \\ ${ }^{13}$ Department of Astronomy and Astrophysics, Pennsylvania State University, 525 Davey Lab, University Park, PA 16802, USA \\ ${ }^{14}$ Department of Physics and Astronomy, University of Southampton, Southampton SO17 1BJ, UK \\ ${ }^{15}$ Lowell Observatory, Flagstaff, AZ 86001, USA \\ ${ }^{16}$ Instituto de Radio Astronomía Milimétrica, Avenida Divina Pastora 7, Local 20, E-18012 Granada, Spain \\ ${ }^{17}$ Maria Mitchell Observatory, 4 Vestal Street, Nantucket, MA 02554, USA \\ ${ }^{18}$ EAPS, MIT, 77 Massachusetts Avenue, Cambridge, MA 02139, USA \\ ${ }^{19}$ Department of Physics and Astronomy, University of California, Los Angeles, CA, USA \\ Received 2013 March 27; accepted 2013 July 5; published 2013 August 5
}

\begin{abstract}
We analyze the multi-frequency behavior of the quasar 3C 454.3 during three prominent $\gamma$-ray outbursts: 2009 Autumn, 2010 Spring, and 2010 Autumn. The data reveal a repeating pattern, including a triple flare structure, in the properties of each $\gamma$-ray outburst, which implies similar mechanism(s) and location for all three events. The multi-frequency behavior indicates that the lower frequency events are co-spatial with the $\gamma$-ray outbursts, although the $\gamma$-ray emission varies on the shortest timescales. We determine that the variability from UV to IR wavelengths during an outburst results from a single synchrotron component whose properties do not change significantly over the different outbursts. Despite a general increase in the degree of optical linear polarization during an outburst, the polarization drops significantly at the peak of the $\gamma$-ray event, which suggests that both shocks and turbulent processes are involved. We detect two disturbances (knots) with superluminal apparent speeds in the parsec-scale jet associated with the outbursts in 2009 Autumn and 2010 Autumn. The kinematic properties of the knots can explain the difference in amplitudes of the $\gamma$-ray events, while their millimeterwave polarization is related to the optical polarization during the outbursts. We interpret the multi-frequency behavior within models involving either a system of standing conical shocks or magnetic reconnection events located in the parsec-scale millimeter-wave core of the jet. We argue that $\gamma$-ray outbursts with variability timescales as short as $\sim 3 \mathrm{hr}$ can occur on parsec scales if flares take place in localized regions such as turbulent cells.
\end{abstract}

Key words: galaxies: active - galaxies: jets - quasars: individual (3C 454.3) - techniques: interferometric techniques: photometric - techniques: polarimetric

\section{INTRODUCTION}

The basic cause of the extremely high nonthermal luminosity and pronounced variability of flux and polarization in the blazar class of active galactic nuclei (AGNs) can be explained through the paradigm of a relativistic jet of high-energy plasma (e.g., Blandford \& Königl 1979; Marscher \& Gear 1985; Sikora et al. 2009). However, our understanding remains limited about the physical processes, such as the compression and heating of the plasma and production of relativistic electrons that generate the emission, as well as the driver behind the rapid fluctuations in the flow speed (and possibly direction), magnetic field, and number of radiating electrons in the jet. Studies of large samples of blazars are valuable for defining the statistics of the observed properties, such as the probability function of the flow velocity and the correlation between apparent velocities of knots in the jet 
and the observed level of $\gamma$-ray emission (e.g., Lister et al. 2011). More detailed observations of individual objects can provide a wealth of information as well, ranging from time profiles of major events (e.g., flares), physical properties of emission features such as knots displaying apparent superluminal motion, and the evolution of well-sampled spectral energy distributions (SEDs) at different times (e.g., Marscher et al. 2010; Jorstad et al. 2010; Agudo et al. 2011a, 2011b). This is especially true when a blazar undergoes a singular event that can be readily identified at different wavebands (Wehrle et al. 2012).

The quasar 3C 454.3 (redshift $z=0.859$ ) is a prime example of a blazar that exhibits such singular events. Villata et al. (2007), Raiteri et al. (2008), Hagen-Thorn et al. (2009), and Jorstad et al. (2010) have analyzed comprehensive multi-waveband observations of an extraordinary radio to X-ray outburst in 2005, as well as major, but less pronounced, flares over the following two years. After the launch of the Astro-rivelatore Gamma a Immagini LEggero (AGILE) and Fermi Gamma-ray Space Telescope orbiting observatories, 3C 454.3 displayed unprecedentedly bright $\gamma$-ray outbursts in late 2009, 2010 April, and late 2010 (Ackermann et al. 2010; Abdo et al. 2011; Bonnoli et al. 2011; Raiteri et al. 2011; Vercellone et al. 2011; Wehrle et al. 2012). During the 2010 event, 3 C 454.3 reached the highest $\gamma$-ray flux ever detected from a single non-transient cosmic source. A variety of telescopes observed contemporaneous outbursts from millimeter wavelengths (mm-wave) to $\gamma$-rays. Analysis of the resulting rich dataset serves as a valuable probe into the structure and physical conditions of the jet at distances within dozens of parsecs from the central engine, as well as the changes in those conditions that cause such an outburst.

Here we perform an analysis of the trio of outbursts from late 2009 to early 2011. We combine observations from mmwave to $\gamma$-ray energies and compare the timing of features in the light curves, polarization variations versus time curves, and mmwave images (from the Very Long Baseline Array, VLBA, in both total and polarized intensity) to provide a comprehensive description of the variations in emission and structure of the jet during the outbursts. The data reveal repeated patterns of variability during the outbursts, implying that the location in the jet and physical conditions are similar for the different events. We are able to infer the location of the emission sites relative to a bright, essentially stationary feature found on the upstream end of the mm-wave images, referred to as the "core." The location constrains the source of seed photons that are scattered to $\gamma$-ray energies.

We present the observations in Section 2, followed by analyses of the data in Sections 3-6. In Section 7 we discuss the implications of the data and offer a physical interpretation of the outbursts. We draw conclusions in Section 8.

\section{OBSERVATIONS AND DATA REDUCTION}

We have used data obtained for the quasar 3C 454.3 from 2009 April 15 to 2011 August 1 from $\gamma$-ray to mm-wave at: (1) $0.1-300 \mathrm{GeV}$, (2) $0.3-10 \mathrm{keV}$, (3) 2030-3501 $\AA$, (4) optical BVRIJHK bands, (5) 4-21 $\mu \mathrm{m}$, (6) $70-500 \mu \mathrm{m}$, (7) $350 \mathrm{GHz}(0.85 \mathrm{~mm}), 230 \mathrm{GHz}(1.3 \mathrm{~mm}), 86.24 \mathrm{GHz}$ (3.5 mm), $43 \mathrm{GHz}(7 \mathrm{~mm})$, and $37 \mathrm{GHz}(8 \mathrm{~mm})$. The observations from optical to $\mathrm{mm}$-wavelengths as well as the data reduction at all wavelengths were performed by the authors. Throughout the paper we use Reduced Julian Date, RJD, which is $\mathrm{RJD}=\mathrm{JD}-2,450,000.0$; the analyzed period in RJD dates is from RJD: 4937.5 to RJD: 5774.5. Current standard cosmological constants with $\Omega_{\mathrm{m}}=0.3, \Omega_{\Lambda}=0.7$, and Hubble constant
$H_{\circ}=71 \mathrm{~km} \mathrm{~s}^{-1} \mathrm{Mpc}^{-1}$ are used in calculations; this gives a scale of $7.7 \mathrm{pc} \mathrm{mas}^{-1}$ at the quasar redshift.

\subsection{Multi-frequency Light Curves}

The $\gamma$-ray data are collected with the Large Area Telescope (LAT) of the Fermi Gamma-ray Space Telescope. We construct a daily $\gamma$-ray light curve of the quasar using Pass 7 photon and spacecraft data, version V9r23pl of the Fermi Science Tools, and the instrument responses for the gal_2yearp7v6_v0 and iso_p7v6clean.txt diffuse source models. We model the $\gamma$-ray emission from 3C 454.3 and other point sources within 15 deg radius of the quasar with spectral models, as found in the 2FGL catalog of sources detected by the LAT. We have fixed the catalog's spectral parameters of sources within the area and searched for values of flux normalization parameters with 1 day integration intervals of photons between 0.1 and $200 \mathrm{GeV}$ using the standard unbinned likelihood analysis. This produces a $\gamma$-ray light curve with 823 measurements, with 59 values representing only upper limits to the $\gamma$-ray emission. The flux is considered detected if the test statistic, TS, provided by the analysis exceeds 10 , which corresponds to approximately a $3 \sigma$ detection level (Nolan et al. 2012).

We have acquired X-Ray Telescope (XRT) and Ultraviolet and Optical Telescope (UVOT) data of 3C 454.3 from 2009 April 25 to 2011 August 1 from the Swift archive and processed them with the HEAsoft version 6.11 software package. We have obtained 201 measurements of the flux at $0.3-10 \mathrm{keV}$. The XRT observations were carried out in a mixture of Photon Counting (PC) and Windowed Timing (WT) modes (Hill et al. 2004). We counted photons with apertures as proposed by Raiteri et al. (2011), a 30 pixel circular region $(\sim 71$ arcsec) and an annular region with inner and outer radii of 110 and 160 pixels for the source and background measurements, respectively. All of the observations collected in PC mode were near or exceeded 0.5 counts $\mathrm{s}^{-1}$, indicating possible photon pile-up that was corrected by eliminating 3-5 central pixels. A new exposure map was generated using the Swift XRT task xrtexpomap. For each observation collected in WT mode, we created a box-shaped extraction region individually sized to exclude the point at which the pixels dropped to less than 2 counts. The Swift XRT task xrtmkarf was applied on all extracted spectra. The spectra were rebinned with the FTOOLS task grppha to include a minimum of 20 photons in each channel. We used XSPEC version 12.7.0 to fit the data with a single powerlaw model while the hydrogen column density was fixed at $1.34 \times 10^{21} \mathrm{~cm}^{-2}$ (Villata et al. 2006).

For the UVOT data, we extracted the magnitude and its error using the tool UVOTSOURCE, specifying a region with a circular aperture of 7 arcsec, and a background annulus region centered on the object with inner and outer radius of 22 and 25 arcsec, respectively. The magnitudes were corrected for Galactic extinction following the procedures outlined in Cardelli et al. (1989), with $A(V)=0.355 \mathrm{mag}$ and $E(B-V)=$ 0.107 mag (Schleger et al. 1998). We converted the magnitudes to fluxes using the central wavelengths for each filter as calibrated by Poole et al. (2008).

The optical photometric data in BVRI bands were collected at various telescopes: (1) the $1.83 \mathrm{~m}$ Perkins telescope of Lowell Observatory (Flagstaff, AZ); (2) the 1.54 m Kuiper and $2.3 \mathrm{~m}$ Bok telescopes of Steward Observatory (Mt. Bigelow and Kitt Peak, AZ); (3) the $70 \mathrm{~cm}$ AZT-8 telescope of the Crimean Astrophysical Observatory (Nauchnij, Ukraine); (4) the $40 \mathrm{~cm}$ LX-200 telescope of St. Petersburg State University 


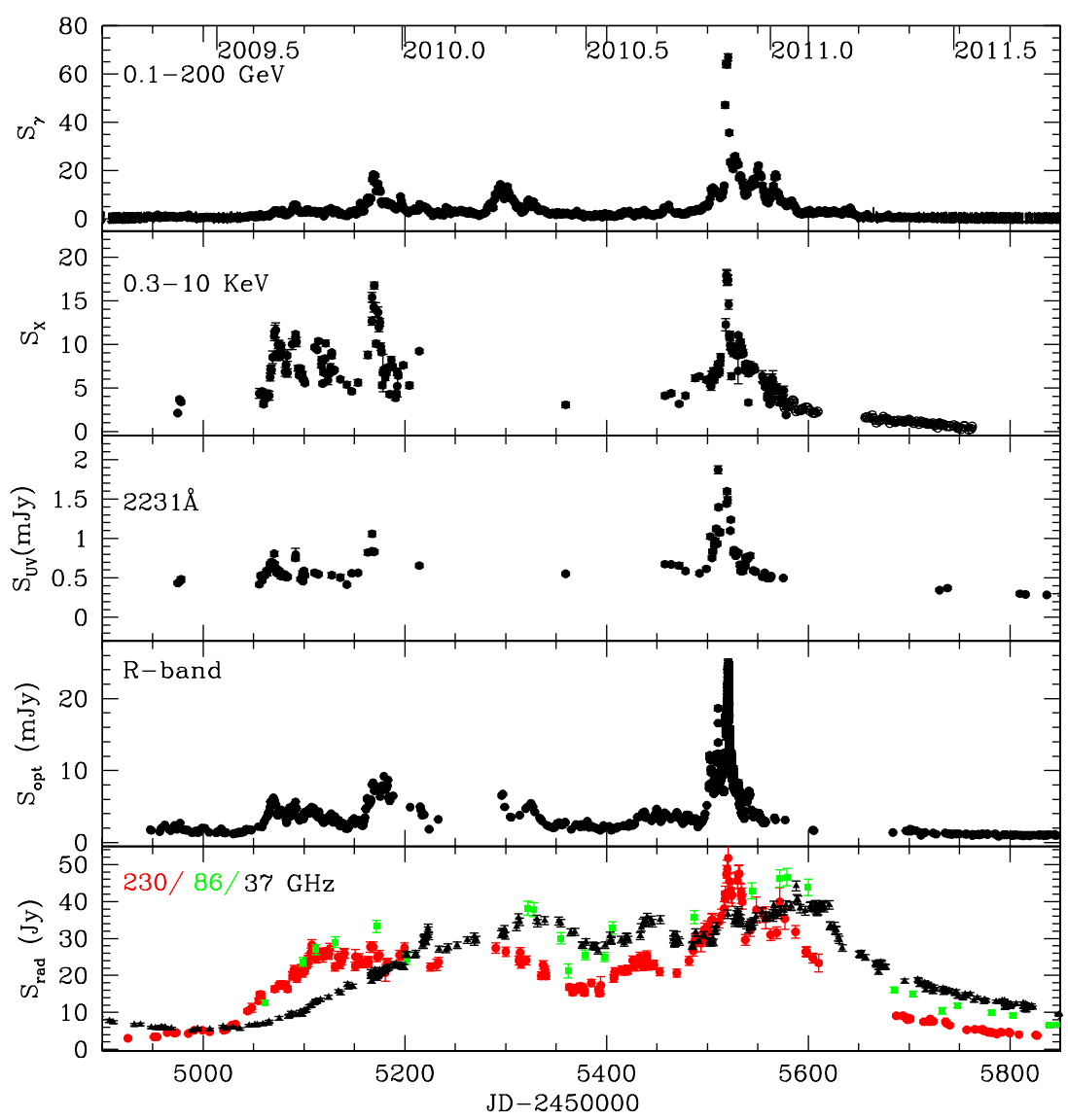

Figure 1. Light curves of the quasar 3C 454.3 at different frequencies. From the top: (1) Fermi LAT $\gamma$-ray flux with 1 day binning interval in units of $10^{-6}$ photons cm $\mathrm{cm}^{-2} \mathrm{~s}^{-1}$; (2) Swift X-ray flux in units of $10^{-11} \mathrm{erg} \mathrm{cm}^{-2} \mathrm{~s}^{-1}$; (3) flux measurements at $2231 \AA$; (4) optical light curve in $R$ band; and (5) flux densities at $230 \mathrm{GHz}(1.3 \mathrm{~mm}$, red circles), $86 \mathrm{GHz}$ ( $3 \mathrm{~mm}$, green squares), and $37 \mathrm{GHz}$ (8mm, black triangles).

(St. Petersburg, Russia); (5) the $2.2 \mathrm{~m}$ telescope of Calar Alto Observatory (Almería, Spain); (6) the $2 \mathrm{~m}$ Liverpool telescope of the Observatorio del Roque de Los Muchachos (Canary Island, Spain); (7) the $1.25 \mathrm{~m}$ telescope of Abastumani Astrophysical Observatory (Mt. Kanobili, Georgia); (8) the $60 \mathrm{~cm}$ telescope of the Maria Mitchell Association (Nantucket, MA); (9) the $1.3 \mathrm{~m}$ telescope at the Cerro Tololo Inter-American Observatory (CTIO), and (10) the UVOT of Swift.

Near-infrared, $J H K$, photometric data (near-IR) were collected at the $1.1 \mathrm{~m}$ telescope of the Main (Pulkovo) Astronomical Observatory of the Russian Academy of Sciences located at Campo Imperatore, Italy (Larionov et al. 2008). The optical and near-IR data were supplemented by measurements by the SMARTS consortium, posted at their website (Bonning et al. 2012). The data have been corrected for Galactic extinction. The conversion factors calculated by Mead et al. (1990) were used to convert magnitudes into flux densities.

Mid-infrared (mid-IR) observations were carried out on 2010 November 3 at the NASA Infrared Telescope Facility (IRTF) with the MIRSI camera (Kassis et al. 2008). The observations were performed in three bands centered at 4.9, 10.6, and $20.7 \mu \mathrm{m}$ with total on-source integration times of 480, 720, and $960 \mathrm{~s}$, and frame times of 200, 24, and $4 \mathrm{~ms}$, respectively. The comparison star $63 \mathrm{Peg}$ from the MIRAC3 Users's Manual was observed before and after each observation of the quasar to provide the flux density calibration. The data were reduced with an IDL script supplied by the IRTF staff. This resulted in flux measurements of $293 \pm 21,699 \pm 37$, and $1293 \pm 197 \mathrm{mJy}$ at $4.9,10.6$, and $20.7 \mu \mathrm{m}$, respectively.
Far-infrared (far-IR) photometric data were collected from 2010 December 25 to 2011 January 10 at 250, 350, and $500 \mu \mathrm{m}$ with the SPIRE photometer (13 measurements at each wavelength) and with the PACS photometer at 70 and $160 \mu \mathrm{m}$ (15 measurements at each wavelength) on board the Herschel satellite. The details of the observations and data reduction along with a table of flux densities can be found in Wehrle et al. (2012).

The $0.85 \mathrm{~mm}(350 \mathrm{GHz})$ and $1.3 \mathrm{~mm}(230 \mathrm{GHz})$ measurements were obtained at the Submillimeter Array (SMA), Mauna Kea, Hawaii, within a monitoring program of compact extragalactic radio sources that can be used as calibrators at $\mathrm{mm}$ and submm wavelengths (Gurwell et al. 2007). The data at $0.85 \mathrm{~mm}$ (47 data points) and $1.3 \mathrm{~mm}$ (215 data points) are supplemented by measurements at the IRAM telescope at $1.3 \mathrm{~mm}$ (22 data points) and $3.5 \mathrm{~mm}$ (28 data points). The data reduction procedure of the IRAM data can be found in Agudo et al. (2010). We will refer to the combined light curve at $1.3 \mathrm{~mm}$ as "the $1 \mathrm{~mm}$ light curve."

The $8 \mathrm{~mm}$ (37 GHz) observations were performed with the $13.7 \mathrm{~m}$ telescope at Metsähovi Radio Observatory of Aalto University, Finland (307 measurements). The flux density calibration is based on observations of DR 21, with 3C 84 and 3C 274 used as secondary calibrators. A detailed description of the data reduction and analysis is given in Teräsranta et al. (1998).

Figure 1 shows the $\gamma$-ray, X-ray, UV, optical $R$-band, and radio light curves from 2009 April 25 to 2011 August 1 (RJD: 4947-5775). Simple visual inspection of the light curves reveals a prolonged, $\sim 600$ day, state of high activity at $\gamma$-rays, 


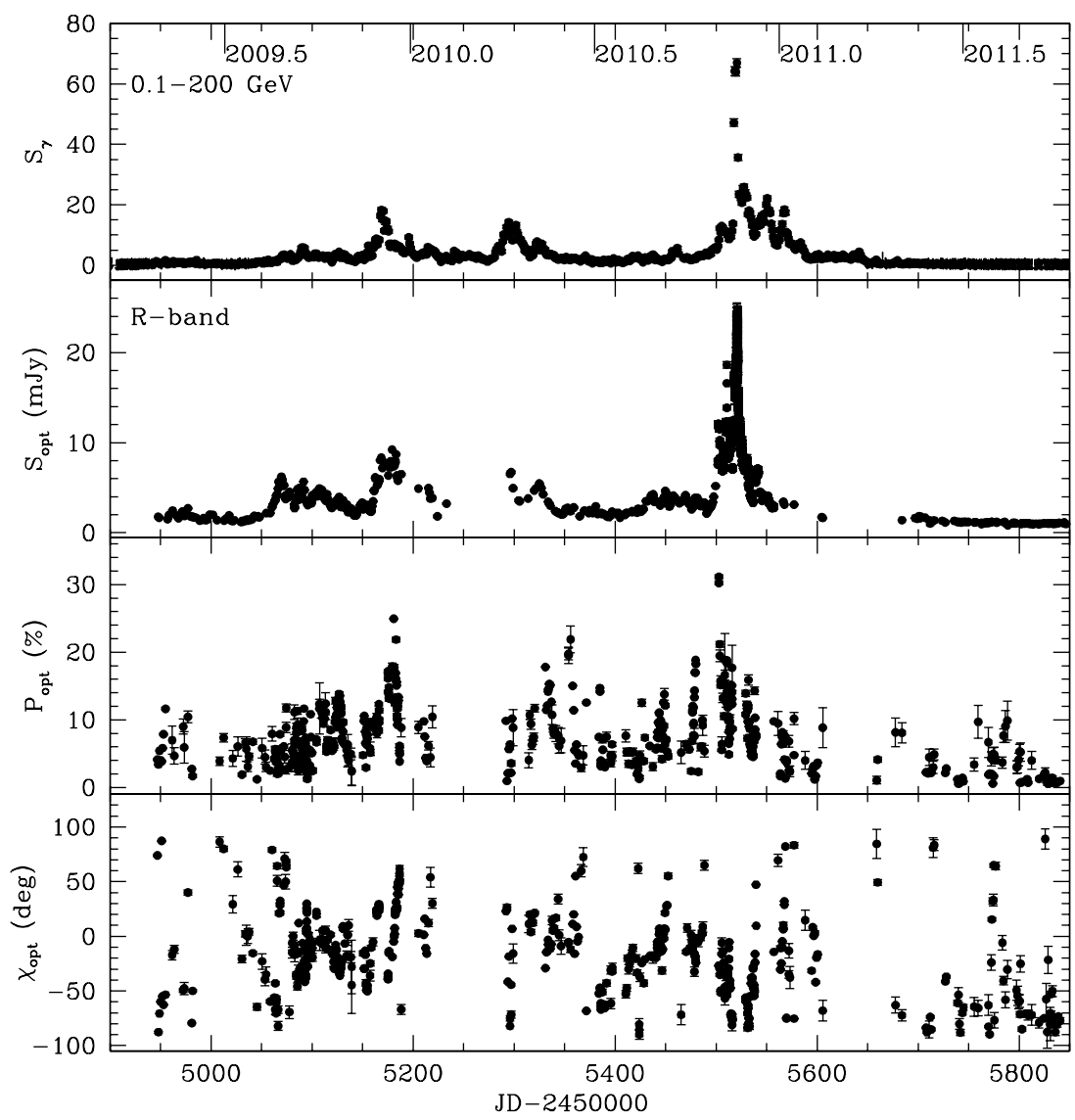

Figure 2. Optical polarization curves of the quasar 3C 454.3 along with the $\gamma$-ray and optical light curves. From the top: (1) $\gamma$-ray light curve with 1 day binning interval in units of $10^{-6}$ photon $\mathrm{cm}^{-2} \mathrm{~s}^{-1}$; (2) optical light curve in $R$ band; (3) degree of optical linear polarization; (4) position angle of optical polarization.

from 2009 August (RJD 5050) to 2011 March (RJD 5650), that coincides with a high state seen in the $1 \mathrm{~mm}$ light curve. Within this active state three major $\gamma$-ray outbursts occurred, in 2009 December, 2010 April, and 2010 November.

\subsection{Observations of Spectrum and Polarization}

The optical polarization measurements were performed at telescopes $1-5$ as listed above in $R$ band, except for LX-200 of St. Petersburg State University, where the observations were carried out without a filter with the central wavelength $\lambda_{\text {eff }} \sim 670 \mathrm{~nm}$, and the spectropolarimetric observations at Steward Observatory (see below). The observations at the Calar Alto Observatory were carried out within the Monitoring AGN with Polarimetry at the Calar Alto Telescopes ${ }^{20}$ program. The details of optical polarization observations and data reduction can be found in Jorstad et al. (2010).

The spectropolarimetric observations of $3 \mathrm{C} 454.3$ at Steward Observatory were part of a currently operating program to monitor bright $\gamma$-ray blazars from the Fermi LAT-monitored blazar list. ${ }^{21}$ The observations were performed using the CCD Imaging/Spectro-polarimeter (SPOL; Schmidt et al. 1992), yielding spectra that span the range of $4000-7550 \AA$ with a dispersion of $4 \AA$ pixel $^{-1}$. Depending on the width of the slit used for the observation, the resolution was typically between 16 and $24 \AA$. The flux density averaged over $5400-5600 \AA$ was scaled to agree with that determined from the synthetic $V$-band photometry performed on the same night. As a result,

\footnotetext{
${ }^{20} \mathrm{http} / / /$ www.iaa.es/ iagudo/research/MAPCAT/MAPCAT.html

$21 \mathrm{http}: / /$ james.as.arizona.edu/ psmith/Fermi
}

181 calibrated spectra of the quasar were obtained during the period from 2009 April 25 to 2011 August 1. In polarization mode, the full-resolution Stokes spectra were obtained to calculate the linear polarization parameters within 5000-7000 (244 spectra). Details of the spectropolarimetric data reduction can be found in Smith et al. (2009). The combined optical polarization data obtained from the telescopes used for this study consist of 523 measurements of the degree, $P$, and position angle, $\chi_{\mathrm{opt}}$, of polarization. The data are displayed in Figure 2.

Polarization observations of the quasar at 1.3 and $3 \mathrm{~mm}$ were obtained at the IRAM 30 m telescope within the MAPI ${ }^{22}$ and POLAMI ${ }^{23}$ polarimetric programs. Each program performs monthly monitoring of a sample of $\gamma$-ray blazars, with both samples including $3 \mathrm{C}$ 454.3. The data were reduced in the same manner as described in Agudo et al. (2010). The values of $P$ at all wavelengths were corrected for statistical bias (Wardle \& Kronberg 1974).

\subsection{VLBA Observations}

We observed 3C 454.3 with the VLBA in the course of a program of monthly monitoring of bright $\gamma$-ray blazars at $43 \mathrm{GHz}(7 \mathrm{~mm})^{24}$ and more dense monitoring during campaigns in 2009 October 12-25, 2010 April 7-20, and 2010 October 31-November 13. Within these campaigns, the quasar was observed three times. During the period from 2009 April to 2011 August, we obtained 35 total and polarized intensity

\footnotetext{
22 MAPI: Monitoring of AGN with Polarimetry at IRAM $30 \mathrm{~m}$.

23 POLAMI: Polarimetric AGN Monitoring at the IRAM $30 \mathrm{~m}$ Telescope.

24 http://www.bu.edu/blazars/VLBAproject.html
} 
images at a resolution of $\sim 0.3 \times 0.1$ mas. We performed the data reduction in the manner of Jorstad et al. (2005) using the Astronomical Image Processing System (AIPS) and Difmap (Shepherd 1997). The electric vector position angle (EVPA) was calibrated by different methods. Over the period 2009 April-2009 December we used the NRAO polarization database $^{25}$ that provides EVPAs at $43 \mathrm{GHz}$ for several sources in our sample (0420-014, 0528+134, OJ287, 1156+295, 3C 279, BL Lacertae, and 3C 454.3) obtained with the Very Large Array (VLA), which we compared with VLBA integrated EVPAs at simultaneous or nearly simultaneous epochs. We obtained polarization measurements during the campaigns with the VLA on 2009 October 14 (sources: 0235+164, 0528+134, BL Lacertae, and 3C 454.3) and with the EVLA on 2010 April 10 (sources: $1156+295,3 \mathrm{C} 279,1308+326$, and OT+081) and on 2010 November 2 (sources: 0235+164, 0528+134, 0716+710, and OJ287). At epochs where VLA/EVLA data were not available, we used the D-terms method (Gómez et al. 2002). The calibration was checked for consistency between epochs by comparing EVPAs of polarized jet features located $\geqslant 1$ mas from the core in $0528+134,3 \mathrm{C} 273,3 \mathrm{C} 345$, CTA102, and BL Lacertae that had stable EVPAs based on VLBA observations with simultaneous VLA/EVLA observations. The accuracy of the EVPA calibration is within 5-10 deg. We have corrected the EVPA values of the core using the most recent estimate of the Faraday rotation measure in the core region of 3C $454.3, \mathrm{RM}=1320 \pm 170 \mathrm{rad} \mathrm{m}^{-2}$ (Algaba et al. 2011). The accuracy of the flux density calibration as revealed by comparison between the VLBA integrated flux and VLA/EVLA flux obtained at simultaneous epochs is within $5 \%$.

\section{STRUCTURE AND TIMESCALES OF THE OUTBURSTS}

We define $\gamma$-ray outbursts using the criterion that the $\gamma$-ray flux, $S_{\gamma}$, calculated with an integration interval of 1 day within the energy range from 0.1 to $200 \mathrm{GeV}$, must exceed $2 \times$ $10^{-6}$ photons $\mathrm{cm}^{-2} \mathrm{~s}^{-1}$ and never drop below this level during the event. Therefore, the duration of a $\gamma$-ray outburst is determined by a period when $S_{\gamma}>2 \times 10^{-6}$ photons $\mathrm{cm}^{-2} \mathrm{~s}^{-1}$. This criterion is arbitrary; however, it agrees with visual inspection of the light curve of 3C 454.3. It defines the three brightest $\gamma$-ray states of the quasar as follows: outburst I from 2009 November 9 to 2010 January 29 (RJD: 5145-5226), outburst II from 2010 March 21 to 2010 May 25 (RJD: 5277-5342), and outburst III from 2010 October 10 to 2011 January 30 (RJD: 5480-5592). We employ the same periods to analyze X-ray and optical outbursts. Unfortunately, during the main part of outburst II the quasar was too close to the Sun, resulting in very limited X-ray and optical observations for this event.

\subsection{Gamma-Ray Outbursts}

We have calculated $\gamma$-ray light curves with a $3 \mathrm{hr}$ integration interval during outbursts I, II, and III using the same approach as described in Section 2.1. This results in 646 (38), 526 (24), and 908 (39) measurements for outbursts I, II, and III, respectively, with the numbers in parentheses being non-detections. We have ignored the non-detections in our analysis since they represent a small fraction of the data. The light curves, presented in Figure 3, are normalized to the maximum flux density of each outburst, with time $t=0$ set to the date of the maximum. Figure 3 shows

\footnotetext{
25 http://www.vla.nrao.edu/astro/calib/polar/
}

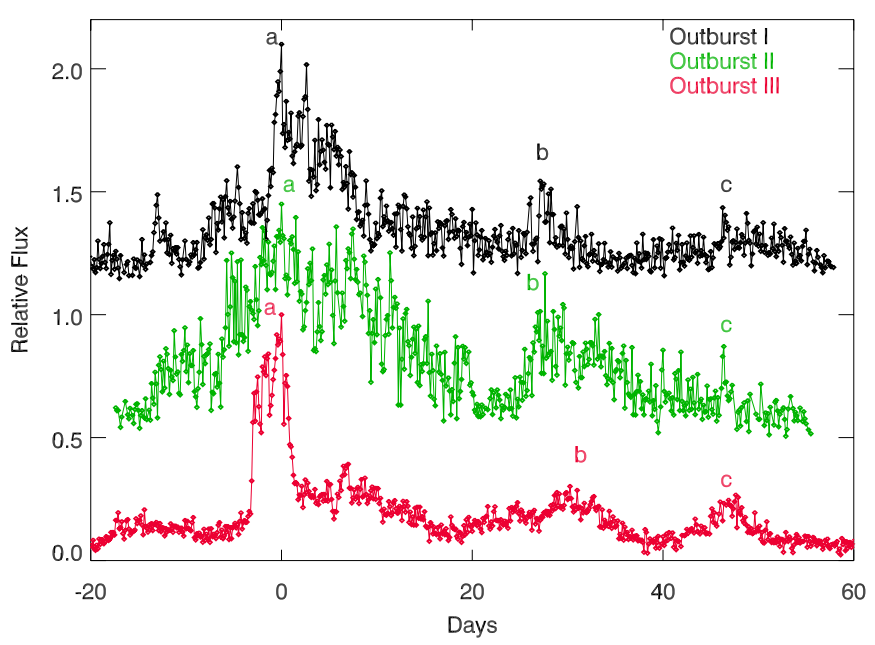

Figure 3. Gamma-ray light curves during outbursts I (black), II (green), and III (red) relative to $T_{\gamma}^{\max }$ of each outburst and normalized to corresponding $S_{\gamma}^{\max }$; light curves I and II are shifted by 1.1 and 0.45 , respectively, for clarity. The three main flares during each outburst are designated as $a, b$, and $c$ (see Table 1).

that the structure of the outbursts is similar, although values of the maximum flux differ. We identify three flares, $a, b$, and $c$, within each outburst, separated by troughs with durations comparable to those of the flares. Peaks $b$ and $c$ have similar delays (within 0.5-3 days) with respect to the main peak of flare $a$. The primary difference in the profiles of the three outbursts is connected with the shape of flare $a$. We define the duration of the main flare, $a$, as the FWHM of a Gaussian that fits the flare profile near maximum flux, $\Delta T_{\gamma}^{\mathrm{a}} \sim 11,20$, and 5 days for outbursts I, II, and III, respectively. All three outbursts have pre-flare and post-flare "plateaus" of enhanced $\gamma$-ray emission, as discussed by Abdo et al. (2011). We determine the duration of a plateau as the time interval within which the standard deviation of the average $\gamma$-ray flux does not exceed $2 \sigma S_{\text {ave }}$, where $\sigma S_{\text {ave }}$ is the average uncertainty of individual measurements. The duration of the plateaus differs from outburst to outburst, although the duration of the pre-flare plateau, $\Delta T_{\gamma}^{\text {pre }}$, is almost equal to the duration of the post-flare plateau, $\Delta T_{\gamma}^{\text {post }}$, for each outburst. In addition, the flux levels of the pre- and post-flare plateaus are comparable, except for outburst III. The entire duration of flare $a$, which includes $\Delta T_{\gamma}^{\mathrm{pre}}, \Delta T_{\gamma}^{\mathrm{a}}$, and $\Delta T_{\gamma}^{\mathrm{post}}$, is comparable for all outbursts $(28,33$, and 31 days for outbursts I, II, and III, respectively), as is the period between the peaks of flares $a$ and $c$, equal to 46, 46, and 48 days for outbursts I, II, and III, respectively. The similarity in structure of the $\gamma$-ray outbursts argues in favor of the same mechanism(s) and location of $\gamma$ ray production for all three events. Jorstad et al. (2010) have previously reported a triple flare structure of optical outbursts in 3C 454.3 that coincide with the time of passage of superluminal knots through the mm-wave core of the jet. The measured time interval between the first and third peaks of these earlier events was $\sim 50$ days, which is only slightly longer than the interval between the peaks of flares $a$ and $c$ observed for $\gamma$-ray outbursts I, II, and III. Parameters of the $\gamma$-ray outbursts studied here are given in Table 1.

We have determined timescales of $\gamma$-ray flux variability, $\tau_{\gamma}$, using the formalism suggested by Burbidge et al. (1974): $\tau \equiv \Delta t / \ln \left(S_{2} / S_{1}\right)$, where $S_{\mathrm{i}}$ is the flux density at epoch $t_{\mathrm{i}}$, with $S_{2}>S_{1}$, and $\Delta t=\left|t_{2}-t_{1}\right|$. We have calculated the timescale of variability for all possible pairs of flux measurements within 3 days of each other if, for a given pair, $S_{2}-S_{1}>3\left(\sigma S_{1}+\sigma S_{2}\right) / 2$, where $\sigma S_{\mathrm{i}}$ is the uncertainty of an individual measurement, and 
Table 1

Parameters of Gamma-Ray Outbursts

\begin{tabular}{|c|c|c|c|}
\hline Parameter & Outburst I & Outburst II & Outburst III \\
\hline$\Delta T_{\gamma}$ (days) & 82 & 66 & 113 \\
\hline$\left\langle S_{\gamma}\right\rangle\left(10^{-6}\right.$ photons $\left.\mathrm{cm}^{-2} \mathrm{~s}^{-1}\right)$ & $5.84 \pm 3.79$ & $5.94 \pm 3.34$ & $12.36 \pm 11.66$ \\
\hline$\left\langle\sigma_{\gamma}\right\rangle\left(10^{-6}\right.$ photons $\left.\mathrm{cm}^{-2} \mathrm{~s}^{-1}\right)$ & 1.04 & 1.05 & 1.87 \\
\hline$\Delta T_{\gamma}^{\mathrm{a}}($ days $)$ & 11 & 20 & 5 \\
\hline$T_{\gamma}^{\max }$ & 2009 Dec 2 & 2010 Apr 8 & 2010 Nov 20 \\
\hline$T_{\gamma}^{\max }(\mathrm{RJD})$ & 5168.343 & 5294.593 & 5520.593 \\
\hline$S_{\gamma}^{\max }\left(10^{-5}\right.$ photons $\left.\mathrm{cm}^{-2} \mathrm{~s}^{-1}\right)$ & $2.41 \pm 0.23$ & $1.60 \pm 0.15$ & $8.38 \pm 0.40$ \\
\hline$\alpha_{\gamma}^{\max }$ & $1.32 \pm 0.04$ & $1.34 \pm 0.05$ & $1.20 \pm 0.02$ \\
\hline$\tau_{\gamma}^{\mathrm{a}}(\mathrm{hr})$ & 5.2 & 4.3 & 5.4 \\
\hline$f_{\gamma}^{\mathrm{a}}$ & 1.79 & 2.02 & 1.74 \\
\hline$\Delta T_{\gamma}^{\mathrm{pre}}$ (days) & 8 & 7 & 13 \\
\hline$S_{\gamma}^{\text {pre }}\left(10^{-6}\right.$ photons $\left.\mathrm{cm}^{-2} \mathrm{~s}^{-1}\right)$ & $6.98 \pm 1.05$ & $5.54 \pm 0.83$ & $11.03 \pm 1.02$ \\
\hline$\alpha_{\gamma}^{\text {pre }}$ & $1.34 \pm 0.10$ & $1.36 \pm 0.11$ & $1.30 \pm 0.06$ \\
\hline$\tau_{\gamma}^{\mathrm{pre}}(\mathrm{hr})$ & 3.9 & 3.1 & 4.4 \\
\hline$f_{\gamma}^{\mathrm{pre}}$ & 2.18 & 2.64 & 1.97 \\
\hline$\Delta T_{\gamma}^{\text {post }}($ days $)$ & 9 & 6 & 13 \\
\hline$S_{\gamma}^{\text {post }}\left(10^{-6}\right.$ photons $\left.\mathrm{cm}^{-2} \mathrm{~s}^{-1}\right)$ & $6.51 \pm 0.92$ & $5.34 \pm 0.89$ & $20.91 \pm 4.20$ \\
\hline$\alpha_{\gamma}^{\text {post }}$ & $1.31 \pm 0.08$ & $1.41 \pm 0.07$ & $1.35 \pm 0.06$ \\
\hline$\tau_{\gamma}^{\text {post }}(\mathrm{hr})$ & 5.6 & 3.5 & 7.3 \\
\hline$f_{\gamma}^{\text {post }}$ & 1.71 & 2.35 & 1.51 \\
\hline$T_{\gamma}^{\mathrm{b}}$ & 2009 Dec 29 & 2010 May 5 & 2010 Dec 20 \\
\hline$T_{\gamma}^{\mathrm{b}}(\mathrm{RJD})$ & 5195.47 & 5322.22 & 5550.84 \\
\hline$S_{\gamma}^{\mathrm{b}}\left(10^{-5}\right.$ photons $\left.\mathrm{cm}^{-2} \mathrm{~s}^{-1}\right)$ & $1.07 \pm 0.16$ & $1.14 \pm 0.16$ & $2.53 \pm 0.22$ \\
\hline$\alpha_{\gamma}^{\mathrm{b}}$ & $1.36 \pm 0.04$ & $1.36 \pm 0.03$ & $1.29 \pm 0.02$ \\
\hline$T_{\gamma}^{\mathrm{c}}$ & 2010 Jan 18 & 2010 May 18 & 2011 Jan 6 \\
\hline$T_{\gamma}^{\mathrm{c}}(\mathrm{RJD})$ & 5214.59 & 5340.59 & 5568.22 \\
\hline$S_{\gamma}^{\mathrm{c}}\left(10^{-6}\right.$ photons $\left.\mathrm{cm}^{-2} \mathrm{~s}^{-1}\right)$ & $8.1 \pm 1.4$ & $6.5 \pm 1.1$ & $22.3 \pm 4.4$ \\
\hline$\alpha_{\gamma}^{\mathrm{c}}$ & $1.38 \pm 0.04$ & $1.32 \pm 0.04$ & $1.26 \pm 0.02$ \\
\hline$\tau_{\gamma}^{\min }(\mathrm{hr})$ & 3.9 & 3.1 & 4.4 \\
\hline$T_{\gamma}^{\tau_{\min }}(\mathrm{RJD})$ & 5161.343 & 5281.593 & 5506.093 \\
\hline$\left\langle\tau_{\gamma, 2}\right\rangle(\mathrm{hr})$ & 19 & 21 & 22 \\
\hline
\end{tabular}

Notes. $\Delta T_{\gamma}$ : duration of $\gamma$-ray outburst (see text Section 3.1 ); $\left\langle S_{\gamma}\right\rangle$ : the average flux density during the outburst and its standard deviation; $\left\langle\sigma_{\gamma}\right\rangle$ : the average $1 \sigma$ uncertainty of an individual measurement during the outburst; $\Delta T_{\gamma}^{\mathrm{a}}$ : duration of the main sub-flare in flare $a(\mathrm{FWHM}) ; S_{\gamma}^{\max }: \gamma$-ray flux at the peak of flare $a$ at $0.1-200 \mathrm{GeV}$ calculated with a $3 \mathrm{hr}$ integration interval; $\alpha_{\gamma}^{\max }$ : spectral energy index at $0.1-200 \mathrm{GeV}$ calculated for a simple power-law model for a 1 day integration interval centered at $T_{\gamma}^{\max } ; \tau_{\gamma}^{\mathrm{a}}$ : minimum timescale of variability of $\gamma$-ray flux during the main flare; $f_{\gamma}^{\mathrm{a}}$ : factor of the $\gamma$-ray flux change over $\tau_{\gamma}^{\mathrm{a}} ; \Delta T_{\gamma}^{\text {pre }}$ : duration of the pre-flare plateau during an $a$ flare; $S_{\gamma}^{\text {pre }}$ : the average $\gamma$-ray flux and its standard deviation over period of $\Delta T_{\gamma}^{\text {pre }} ; \alpha_{\gamma}^{\text {pre }}$ : spectral index at $0.1-200 \mathrm{GeV}$ averaged over $\Delta T_{\gamma}^{\mathrm{pre}} ; \tau_{\gamma}^{\mathrm{pre}}$ : minimum timescale of variability of $\gamma$-ray flux during $\Delta T_{\gamma}^{\mathrm{pre}} ; f_{\gamma}^{\mathrm{pre}}$ : factor of the $\gamma$-ray flux change over $\tau_{\gamma}^{\text {pre }} ; \Delta T_{\gamma}^{\text {post }}, S_{\gamma}^{\text {post }}, \alpha_{\gamma}^{\text {post }}, \tau_{\gamma}^{\text {post }}$, and $f_{\gamma}^{\text {post }}$ : parameters for the post-flare plateau obtained in the same manner as for the pre-flare plateau; $T_{\gamma}^{\mathrm{b}}, S_{\gamma}^{\mathrm{b}}$, and $\alpha_{\gamma}^{\mathrm{b}}$ : epoch, maximum flux, and spectral index, respectively, for flare $b$, calculated in the same manner as for flare $a ; T_{\gamma}^{\mathrm{c}}, S_{\gamma}^{\mathrm{c}}$, and $\alpha_{\gamma}^{\mathrm{c}}$ are epoch, maximum flux, and spectral index, respectively, for flare $c ; \tau_{\gamma}^{\min }$ : minimum timescale of variability of $\gamma$-ray flux during an outburst; $T_{\gamma}^{\tau}$ : epoch of the start of an event with minimum timescale of variability; $\left\langle\tau_{\gamma, 2}\right\rangle$ : typical timescale of flux doubling (see text).

if the test statistic of the $\gamma$-ray measurement TS $>25$ for both measurements. Using the derived values of $\tau_{\gamma}$, we have searched for a minimum timescale of variability among pairs. Table 1 gives minimum timescales of $\sim 3-4 \mathrm{hr}$ with the $\gamma$-ray flux changing by a factor of $\geqslant 2$. Table 1 shows that a short timescale of variability can occur at different stages of an outburst, although the occurrence of $\tau_{\gamma}^{\min }$ takes place during the preflare plateau for all three outbursts. The range is consistent with the minimum timescales of variability reported by Ackermann et al. (2010) and Abdo et al. (2011), as well as Foschini et al. (2011), who apply different methods for estimation of $\tau_{\gamma}$. Note that Foschini et al. (2011) have calculated $\gamma$-ray light curves using a time bin equal to the good time interval (GTI). Such a method should produce the most accurate flux estimates at short timescales for observations performed in scanning mode, since it allows one to find shortest intervals for binning with a sufficient number of photons for a good statistic. Agreement between our results suggests that $\tau_{\gamma}^{\min } \sim 3-5 \mathrm{hr}$ might be an upper limit 


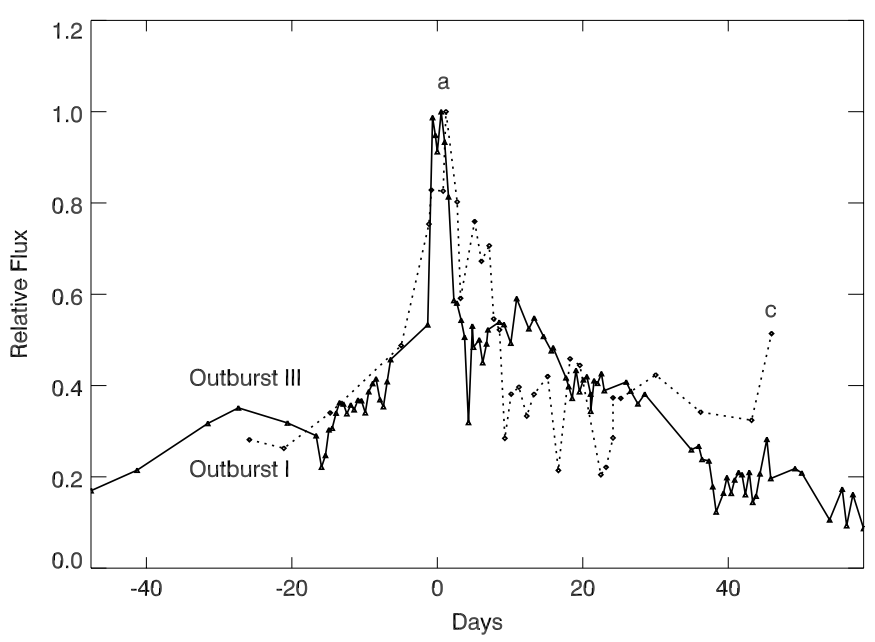

Figure 4. X-ray light curves during outbursts I (diamonds, dotted line) and III (triangles, solid line) normalized to the corresponding maximum and centered with respect to the corresponding peak of $\gamma$-ray outburst.

for the minimum timescale of variability defined by the GTI. In addition, Foschini et al. (2011) report the time of the global peak of outburst III to be RJD: 5520.573-5520.627, which matches $T_{\gamma}^{\max }$ very well. We have also analyzed the distribution of $\tau_{\gamma}$ values that fall within the range $0-72 \mathrm{hr}$ to determine a typical timescale, $\tau_{\gamma, 2}$, for the $\gamma$-ray emission to change by a factor of $\geqslant 2$. Table 1 shows that this timescale of variability is similar for all three outbursts, $\tau_{\gamma, 2}=20 \pm 1 \mathrm{hr}$.

\subsection{X-Ray Outbursts}

Figure 4 displays the X-ray light curves for outbursts I and III normalized to the maximum flux density of each X-ray outburst and centered with respect to the date of the maximum of corresponding $\gamma$-ray outburst. Table 2 lists the parameters of the X-ray outbursts. Although the X-ray data are much more sparsely sampled than the $\gamma$-ray light curves, the global X-ray and $\gamma$-ray peaks of outbursts I and III coincide within $\sim 1$ day, with the $\gamma$-ray peak of outburst I leading by $\sim 1.2$ day while the $\gamma$-ray peak of outburst III is delayed by $\sim 1.0$ day. In addition, the duration of the main X-ray event in flare $a$ is similar to $\Delta T_{\gamma}^{\text {a for }}$ both outbursts, and flare $a$ of outburst III has pre-flare and postflare plateaus contemporaneous with their $\gamma$-ray counterparts. There are also indications of the presence of a post-flare plateau and flare $c$ in outburst I (the durations of the plateaus were determined in the same manner as for the $\gamma$-ray events). The main difference between the X-ray and $\gamma$-ray outbursts is the timescale of variability $\tau_{\mathrm{X}}$, calculated in the same manner as $\tau_{\gamma}$, except for the condition for the test statistic. The fastest events were observed when the X-ray flux changed by a factor of 1.8 in $27 \mathrm{hr}$, which corresponds to $\tau_{\mathrm{X}}^{\min } \simeq 6 \tau_{\gamma}^{\mathrm{min}}$, and the typical flux doubling timescale is $\sim 2$ days, which gives $\tau_{\mathrm{X}, 2} \simeq 2 \tau_{\gamma, 2}$

\subsection{Optical Outbursts}

We perform the same analysis of the structure of the $R$-band optical light curves (Figure 5 and Table 3 ) during outbursts I, II, and III as for the $\gamma$-ray and X-ray light curves. Figure 5 shows that the two well-sampled optical outbursts, I and III, have a complex structure of the main flare, $a$ : flare $a$ of outburst I has 2 peaks, $a 1$ and $a 2$ (Figure 5, the top insert), and flare $a$ of outburst III has at least three peaks, $a 1, a 2$, and $a 3$ (the bottom insert in Figure 5). Remarkably, flare $a$ at $\gamma$-ray energies
Table 2

Parameters of X-ray Outbursts

\begin{tabular}{lcc}
\hline \hline Parameter & Outburst I & Outburst III \\
\hline$M$ & 33 & 96 \\
$\left\langle S_{\mathrm{X}}\right\rangle\left(10^{-11} \mathrm{erg} \mathrm{cm}^{-2} \mathrm{~s}^{-1}\right)$ & $8.46 \pm 3.54$ & $7.50 \pm 3.26$ \\
$\left\langle\sigma_{\mathrm{X}}\right\rangle\left(10^{-11} \mathrm{erg} \mathrm{cm}^{-2} \mathrm{~s}^{-1}\right)$ & 0.45 & 0.40 \\
$\Delta T_{\mathrm{X}}^{\mathrm{a}}($ days $)$ & 13.5 & 5 \\
$T_{\mathrm{X}}^{\max }$ & $2009 \mathrm{Dec} 4$ & 2010 Nov 19 \\
$T_{\mathrm{X}}^{\max }(\mathrm{RJD})$ & 5169.531 & 5519.594 \\
$S_{\mathrm{X}}^{\max }\left(10^{-11} \mathrm{erg} \mathrm{cm}^{-2} \mathrm{~s}^{-1}\right)$ & $16.73 \pm 0.40$ & 24 \\
$\Delta T_{\mathrm{X}}^{\text {pre }}($ days $)$ & $\ldots$ & $6.48 \pm 0.64$ \\
$S_{\mathrm{X}}^{\text {pre }}\left(10^{-11} \mathrm{erg} \mathrm{cm}^{-2} \mathrm{~s}^{-1}\right)$ & $\ldots$ & 12 \\
$\Delta T_{\mathrm{X}}^{\text {post }}($ days $)$ & 7 & $7.85 \pm 0.71$ \\
$S_{\mathrm{X}}^{\text {post }}\left(10^{-11} \mathrm{erg} \mathrm{cm}^{-2} \mathrm{~s}^{-1}\right)$ & $6.61 \pm 0.68$ & 26.2 \\
$\tau_{\mathrm{X}}^{\min }(\mathrm{hr})$ & 33.1 & 1.80 \\
$f_{\mathrm{X}}(\mathrm{hr})$ & 1.71 & 5518.28 \\
$T_{\mathrm{X}}^{\tau_{\min }}(\mathrm{RJD})$ & 5176.89 & 57 \\
$\left\langle\tau_{\mathrm{X}, 2}\right\rangle(\mathrm{hr})$ & 48 & \\
\hline
\end{tabular}

Notes. $M$ : number of X-ray measurements at $0.3-10 \mathrm{keV}$ obtained during the outburst; $\left\langle S_{\mathrm{X}}\right\rangle$ : the average flux density during the outburst and its standard deviation; $\left\langle\sigma_{\mathrm{X}}\right\rangle$ : the average $1 \sigma$ uncertainty of an individual measurement during the outburst; $\Delta T_{\mathrm{X}}^{\mathrm{a}}$ : duration of the main sub-flare in flare $a(\mathrm{FWHM}) ; T_{\mathrm{X}}^{\max }$ : epoch of the global maximum; $S_{\mathrm{X}}^{\max }$ : the flux density at the peak of flare $a ; \Delta T_{\mathrm{X}}^{\text {pre }}$ : duration of the pre-flare plateau during an $a$ flare, the values in parentheses indicating the average uncertainty of an individual measurement of the flux density in $10^{-11} \mathrm{erg} \mathrm{cm}^{-2} \mathrm{~s}^{-1} ; S_{\mathrm{X}}^{\text {pre }}$ : the average flux density and its standard deviation over period of $\Delta T_{\mathrm{X}}^{\text {pre }} ; \Delta T_{\mathrm{X}}^{\text {post }}$ and $S_{\mathrm{X}}^{\text {post }}$ : similar parameters for the post-flare plateau; $\tau_{X}^{\min }$ : minimum timescale of variability of X-ray flux during an outburst; $f_{\mathrm{X}}$ : factor of the flux change over $\tau_{\mathrm{X}}^{\min } ; T_{\mathrm{X}}^{\tau_{\min }}$ : epoch of the start of an event exhibiting the minimum timescale of variability; $\left\langle\tau_{\mathrm{X}, 2}\right\rangle$ : typical timescale of flux doubling.

possesses similar structure for both outbursts, with the global $\gamma$-ray peak ( $a 1$ for outburst I and $a 3$ for outburst III) coinciding with a prominent optical counterpart within the $3 \mathrm{hr} \gamma$-ray sampling, although the ratio of the fluxes of the $\gamma$-ray peaks can be different from those at optical wavelengths. The similarity in structure of flare $a$ at optical and $\gamma$-ray frequencies implies that the flaring emission at the two wavelengths originates in the same region. The difference between the relative amplitudes of $\gamma$-ray versus optical peaks can be explained as the result of differences in relativistic boosting of $\gamma$-ray and optical emission, as proposed by Raiteri et al. (2011), or by variations in the density of seed photons available for scattering to $\gamma$-ray energies, as suggested by Vercellone et al. (2011). The latter is additionally supported by existence of orphan optical outbursts, for example, a very sharp spike at $\sim 10$ days before the maximum when the fastest optical variability during outburst III was observed (Table 3) without an obvious counterpart in the $\gamma$-ray light curve (Figure 5, the bottom insert).

Figure 5 and Table 3 show that optical outbursts I and III have pre-flare and post-flare plateaus that are contemporaneous with the corresponding $\gamma$-ray plateaus, although the relative flux level of the pre-flare plateau is higher with respect to the global maximum at optical wavelengths than at $\gamma$-ray energies. The durations of the optical plateaus are similar to the $\gamma$-ray values. The durations of the plateaus in $R$ band were determined by the criterion that the flux variations within a plateau should not exceed $30 \%$ of the average flux value. This criterion is similar to that used for the $\gamma$-ray data analysis, since the average $1 \sigma$ uncertainty of a $\gamma$-ray measurement is $\sim 17 \%$ (Table 1 ) while 


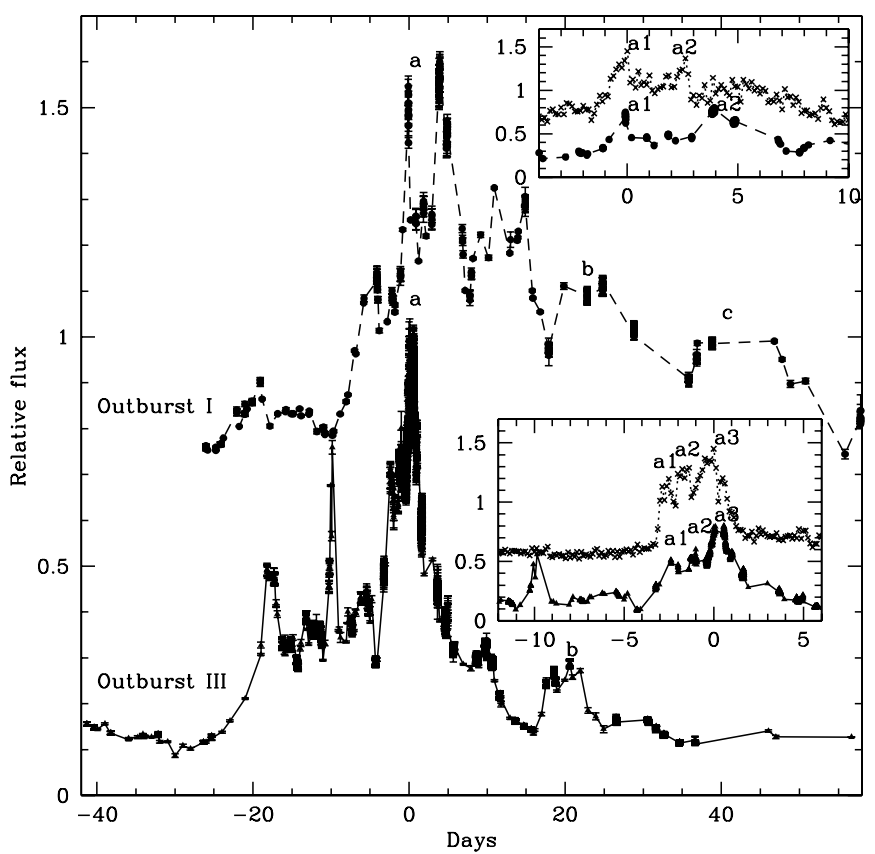

Figure 5. Optical $R$-band light curves during outbursts I (circles, dash line) and III (triangles, solid line) normalized to the corresponding maximum and centered with respect to the corresponding peak of $\gamma$-ray outbursts. The three main flares during each outburst are designated as $a, b$, and $c$ (see Table 3); optical light curves I and III are shifted by 0.6 and -0.2 , respectively, for clarity. The top insert shows the structure of flare $a$ at optical (circles, dash line) and $\gamma$-ray (crosses, dotted line) wavelengths for outburst I; the bottom insert shows the structure of flare $a$ at optical (triangles, solid line) and $\gamma$-ray (crosses, dotted line) wavelengths for outburst III; the $\gamma$-ray fluxes are calculated with a $3 \mathrm{hr}$ binning interval.

the $1 \sigma$ uncertainty of an optical flux is $\sim 2 \%$ (Table 3 ). Note that the duration of the pre-flare plateau of outburst III is a factor of 2 shorter than $\Delta T_{\gamma}^{\text {pre }}$. However, $\Delta T_{\mathrm{opt}}^{\mathrm{pre}}$ would match $\Delta T_{\gamma}^{\text {pre }}$ if the pre-flare plateau were not interrupted by the orphan optical flare mentioned above. For outbursts I and III the entire optical flare $a\left(\Delta T_{\mathrm{opt}}^{\mathrm{a}}+\Delta T_{\mathrm{opt}}^{\mathrm{pre}}+\Delta T_{\mathrm{opt}}^{\mathrm{post}}\right)$ has a similar duration as its $\gamma$-ray counterpart. The post-flare optical variability does not correspond as closely to the $\gamma$-ray variations as during the preflare and main flare stages. Nevertheless, optical outbursts I and II have counterparts to $\gamma$-ray flares $b$ and $c$ (see Table 3 and Figure 5) that peak within 0.5-5 days of the corresponding $\gamma$-ray flares. Flare $b$ is distinct during outburst III as well, although it precedes the $\gamma$-ray flare $b$ by $\sim 9$ days.

Table 3 shows that the minimum timescale of variability is $\sim 18-24 \mathrm{hr}$ with the optical flux changing by a factor of 1.5-2.5 (see also Raiteri et al. 2011; Vercellone et al. 2011). Comparison of Tables 1-3 reveals that the timescale of optical variability is different from $\tau_{\gamma}$ and similar to $\tau_{\mathrm{X}}$, which is longer by a factor of five than the minimum timescale of the $\gamma$-ray flux. Note that during flare $a$ of outburst III (from RJD: 5500 to 5540) we obtained $\sim 2400$ measurements in $R$ band, which is suitable for revealing a timescale of variability as short as $<1 \mathrm{hr}$. The typical doubling timescale is also different for $\gamma$-ray and optical variations, with $\tau_{\mathrm{opt}, 2} \approx(2-3) \tau_{\gamma, 2}$.

\subsection{Millimeter-wave Outbursts}

Parameters of outbursts I and III at $1 \mathrm{~mm}$ are presented in Table 4. Figure 6 shows the structure of outbursts I and III with respect to $T_{\gamma}^{\max }$ of the corresponding $\gamma$-ray outburst. (Unfortunately, as in the case of the X-ray and optical light
Table 3

Parameters of Optical Outbursts

\begin{tabular}{|c|c|c|c|}
\hline Parameter & Outburst I & Outburst II & Outburst III \\
\hline$M$ & 306 & 103 & 2767 \\
\hline$\left\langle S_{\text {opt }}\right\rangle(\mathrm{mJy})$ & $6.49 \pm 3.08$ & $3.68 \pm 1.43$ & $10.93 \pm 5.72$ \\
\hline$\left\langle\sigma_{\mathrm{opt}}\right\rangle(\mathrm{mJy})$ & 0.14 & 0.07 & 0.19 \\
\hline$\Delta T_{\mathrm{opt}}^{\mathrm{a}}$ (days) & 10 & $\ldots$ & 8 \\
\hline$T_{\mathrm{opt}}^{\max }$ & 2009 Dec 6 & 2010 Apr 10 & 2010 Nov 20 \\
\hline$T_{\mathrm{opt}}^{\max }(\mathrm{RJD})$ & 5172.273 & 5297.010 & 5520.673 \\
\hline$S_{\mathrm{opt}}^{\mathrm{max}}(\mathrm{mJy})$ & $12.71 \pm 0.19$ & $6.71 \pm 0.14$ & $24.40 \pm 1.10$ \\
\hline$\Delta T_{\text {opt }}^{\text {pre }}$ (days) & 6 & $\ldots$ & 7 \\
\hline$S_{\mathrm{opt}}^{\mathrm{pre}}(\mathrm{mJy})$ & $6.29 \pm 0.59$ & $\ldots$ & $8.30 \pm 0.72$ \\
\hline$\Delta T_{\mathrm{opt}}^{\mathrm{post}}$ (days) & 10 & $\ldots$ & 6 \\
\hline$S_{\mathrm{opt}}^{\mathrm{post}}(\mathrm{mJy})$ & $7.57 \pm 0.99$ & $\ldots$ & $7.42 \pm 0.34$ \\
\hline$T_{\mathrm{opt}}^{\mathrm{b}}(\mathrm{RJD})$ & 5193.153 & 5324.521 & 5541.180 \\
\hline$S_{\mathrm{opt}}^{\mathrm{b}}(\mathrm{mJy})$ & $6.62 \pm 0.15$ & $6.60 \pm 0.10$ & $7.12 \pm 0.15$ \\
\hline$T_{\mathrm{opt}}^{\mathrm{c}}(\mathrm{RJD})$ & 5215.144 & 5338.522 & $\ldots$ \\
\hline$S_{\text {opt }}(\mathrm{mJy})$ & $4.97 \pm 0.05$ & $3.46 \pm 0.06$ & $\ldots$ \\
\hline$\tau_{\mathrm{opt}}^{\min }(\mathrm{hr})$ & 18.3 & $\ldots$ & 23.7 \\
\hline$f_{\text {opt }}(\mathrm{hr})$ & 1.44 & $\ldots$ & 2.57 \\
\hline$T_{\mathrm{opt}}^{\tau_{\min }}(\mathrm{RJD})$ & 5168.26 & $\ldots$ & 5509.55 \\
\hline$\left\langle\tau_{X, 2}\right\rangle(h r)$ & 58 & $\ldots$ & 40 \\
\hline
\end{tabular}

Notes. $M$ : number of optical measurements in $R$ band obtained during the outburst; $\left\langle S_{\text {opt }}\right\rangle$ : the average flux density during the outburst and its standard deviation; $\left\langle\sigma_{\text {opt }}\right\rangle$ : the average $1 \sigma$ uncertainty of an individual measurement during the outburst; $\Delta T_{\mathrm{opt}}^{\mathrm{a}}$ : duration of the main sub-flare in flare $a$ (FWHM); $S_{\mathrm{opt}}^{\max }$ : the flux density in $R$ band at the peak of flare $a ; \Delta T_{\mathrm{opt}}^{\mathrm{pre}}$ : duration of the preflare plateau during an $a$ flare; $S_{\mathrm{opt}}^{\mathrm{pre}}$ : the average flux in $R$ band and its standard deviation over period of $\Delta T_{\mathrm{opt}}^{\mathrm{pre}} ; \Delta T_{\mathrm{opt}}^{\mathrm{post}}$ and $S_{\mathrm{opt}}^{\text {post }}$ : parameters for the post-flare plateau obtained in the same manner as for the pre-flare plateau; $T_{\mathrm{opt}}^{\mathrm{b}}$ and $S_{\mathrm{opt}}^{\mathrm{b}}$ : epoch and maximum flux, respectively, for flare $b ; T_{\mathrm{opt}}^{\mathrm{c}}$ and $S_{\mathrm{opt}}^{\mathrm{c}}$ : epoch and maximum flux, respectively, for flare $c ; \tau_{\mathrm{opt}}^{\mathrm{min}}$ : minimum timescale of variability of optical flux during an outburst; $f_{\mathrm{opt}}$ : factor of the flux change over $\tau_{\mathrm{opt}}^{\min } ; T_{\mathrm{opt}}^{\tau_{\mathrm{min}}}$ : epoch of the start of an event with minimum timescale of variability; $\left\langle\tau_{\mathrm{opt}, 2}\right\rangle$ : typical timescale of flux doubling (see text).

curves, observations at $1 \mathrm{~mm}$ miss outburst II.) Strikingly, the global peaks of the mm-wave and $\gamma$-ray outbursts coincide within hours for both events, while during the dramatic outburst in 2005 the global peak at $1 \mathrm{~mm}$ was delayed with respect to that at optical wavelengths by $\sim 2$ months (Raiteri et al. 2008; Jorstad et al. 2010). Moreover, the duration of flare $a$ is similar at mmwaves and $\gamma$-rays for both outbursts, and pre-flare and post-flare plateaus are apparent for outburst III. In addition, both outbursts at $1 \mathrm{~mm}$ contain flare $b$, which coincides with the corresponding flare $b$ at $\gamma$-rays within 2 days, and flare $c$ is seen in the $1 \mathrm{~mm}$ light curve of outburst III only 3.5 days later than $\gamma$-ray flare $c$. Taking into account the dramatic difference in the opacity at $\gamma$ and $\mathrm{mm}$ wavelengths, such similarity requires the $\gamma$-ray events to take place in a region that is optically thin at $1 \mathrm{~mm}$. The main differences between the $\gamma$-ray and mm-wave events are connected with the amplitude and timescale of variability. According to Tables 1 and 4, the size of the emission region at $1 \mathrm{~mm}$ is $\sim 100-300$ times larger than that at $\gamma$-rays.

\subsection{Correlation Analysis}

We perform a discrete cross-correlation analysis between the $\gamma$-ray and optical light curves and between the X-ray and optical light curves. For the purpose of this analysis, we construct a 


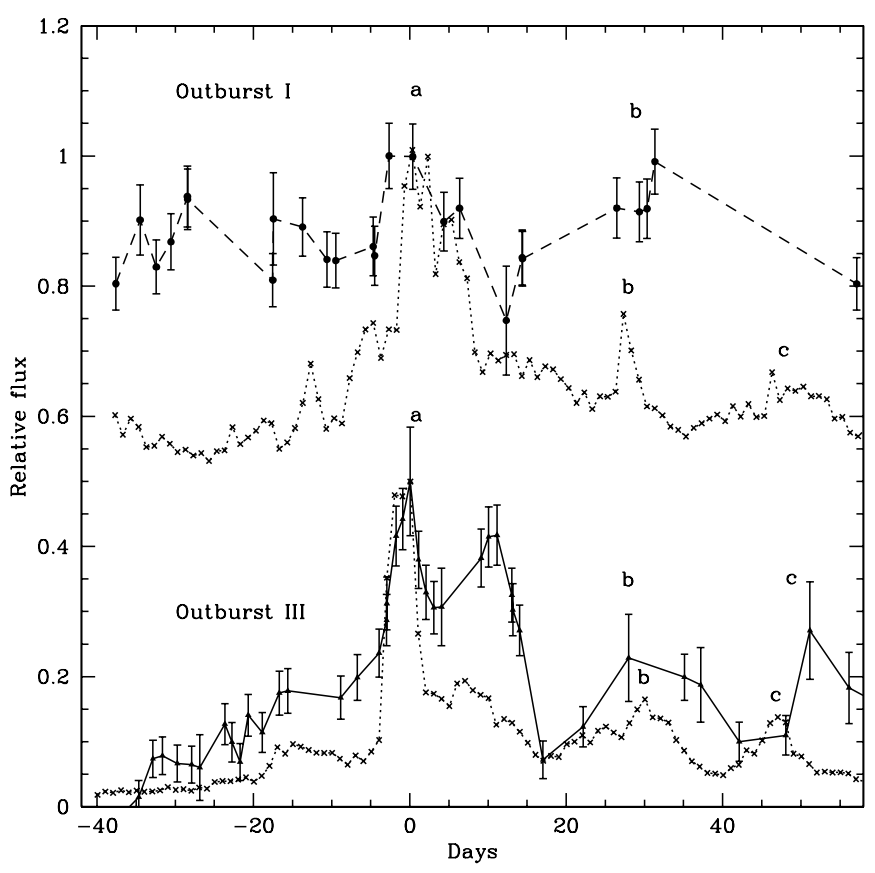

Figure 6. Light curves at $1 \mathrm{~mm}$ during outbursts I (circles, dash line) and III (triangles, solid line), normalized to the corresponding maximum and superposed with the corresponding $\gamma$-ray light curves (crosses, dotted lines). The $\gamma$-ray light curves are normalized to twice the value of the corresponding maximum. All light curves are centered with respect to the corresponding peak of the $\gamma$-ray outbursts. The three main flares during each outburst are designated as $a, b$, and $c$. For clarity, the $\gamma$-ray light curve during outburst I is shifted by +0.5 , while during outburst III the $1 \mathrm{~mm}$ light curve is shifted by -0.5 . The $\gamma$-ray fluxes are calculated with a 1 day binning interval.

$\gamma$-ray light curve with an integration time of $12 \mathrm{hr}$ in the same manner as described in Section 2.1. We use the original sampling of the X-ray light curve, which corresponds to a minimum time interval between two measurements of $\sim 12 \mathrm{hr}$, and bin the optical light curve with a $12 \mathrm{hr}$ minimum interval, although the light curves have gaps ranging from days to months. We calculate the discrete cross-correlation function (DCF) using the algorithm developed by Edelson \& Krolik (1988), and determine the significance of the correlation with the approach suggested by Chatterjee et al. (2008) and Max-Moerbeck et al. (2010). We follow Timmer \& Koenig (1995) by simulating 5000 light curves with the same mean and standard deviation as the observed light curves. The statistics of the flux variations are described by a power spectral density, PSD, with a power-law shape, PSD $\propto f^{-b}$, where a different value of $b$ from 1 to 2.5 , in
Table 4

Parameters of Outbursts at $1 \mathrm{~mm}$

\begin{tabular}{lcc}
\hline \hline Parameter & Outburst I & Outburst III \\
\hline$M$ & 25 & 44 \\
$\left\langle S_{\mathrm{mm}}\right\rangle(\mathrm{Jy})$ & $22.32 \pm 4.27$ & $36.27 \pm 7.04$ \\
$\left\langle\sigma_{\mathrm{mm}}\right\rangle(\mathrm{Jy})$ & 1.17 & 1.63 \\
$\Delta T_{\mathrm{mm}}^{\mathrm{a}}$ (days) & 14 & 6 \\
$T_{\mathrm{mm}}^{\max }$ & $2009 \mathrm{Dec} 3$ & $2010 \mathrm{Nov} 20$ \\
$T_{\mathrm{mm}}^{\max }(\mathrm{RJD})$ & 5168.726 & 5520.657 \\
$S_{\mathrm{mm}}^{\max }($ Jy) & $27.70 \pm 1.39$ & $51.77 \pm 4.31$ \\
$T_{\mathrm{mm}}^{\mathrm{b}}(\mathrm{RJD})$ & 5199.668 & 5548.587 \\
$S_{\mathrm{mm}}^{\mathrm{b}}$ (mJy) & $27.49 \pm 1.38$ & $37.73 \pm 3.46$ \\
$T_{\mathrm{mm}}^{\mathrm{c}}(\mathrm{RJD})$ & $\ldots$ & 5571.764 \\
$S_{\mathrm{mm}}^{\mathrm{c}}$ (mJy) & $\ldots$ & $39.91 \pm 3.88$ \\
$\tau_{\mathrm{mm}}^{\mathrm{min}}($ days $)$ & 41 & 10 \\
$f_{\mathrm{mm}}$ (days) & 1.34 & 1.35 \\
$T_{\mathrm{mm}}^{\tau_{\mathrm{min}}}$ (RJD) & 5168.726 & 5534.64 \\
\hline
\end{tabular}

Notes. $M$ : number of measurements at $1 \mathrm{~mm}$ obtained during the outburst; $\left\langle S_{\mathrm{opt}}\right\rangle$ : the average flux density during the outburst and its standard deviation; $\left\langle\sigma_{\text {opt }}\right\rangle$ : the average $1 \sigma$ uncertainty of an individual measurement during the outburst; $\Delta T_{\mathrm{mm}}^{\mathrm{a}}$ : duration of flare $a(\mathrm{FWHM}) ; T_{\mathrm{mm}}^{\max }$ : epoch of the global maximum; $S_{\mathrm{mm}}^{\max }$ : the flux density at the peak of flare $a ; T_{\mathrm{mm}}^{\mathrm{b}}$ and $S_{\mathrm{mm}}^{\mathrm{b}}$ : epoch and maximum flux, respectively, for flare $b ; T_{\mathrm{mm}}^{\mathrm{c}}$ and $S_{\mathrm{mm}}^{\mathrm{c}}$ : epoch and maximum flux, respectively, for flare $c ; \tau_{\mathrm{mm}}^{\min }$ : minimum timescale of flux variability during an outburst; $f_{\mathrm{mm}}$ : factor of the flux change during $\tau_{\mathrm{mm}}^{\mathrm{min}} ; T_{\mathrm{mm}}^{\tau_{\min }}$ : epoch of the start of an event exhibiting the minimum timescale of variability.

steps of 0.2 , is adopted for each set of simulations (Chatterjee et al. 2012). Figure 7 shows the DCF between the $\gamma$-ray and optical light curves (left) and between the X-ray and optical light curves (right). The DCF between the $\gamma$-ray and optical light curves is symmetric within \pm 2 days of the peak, with no delay between variations at two wavelengths $>12 \mathrm{hr}$, since such a delay would produce, at least, an asymmetry in the DCF peak. The peak is significant at a level $>99.7 \%$. The DCF between the X-ray and optical light curves has a peak near zero delay as well; however, the position of the maximum of the centroid calculated for points exceeding $99.7 \%$ significance gives a delay of the X-ray with respect to optical variations of $0.5 \pm 1$ days. This result is consistent with the finding of Raiteri et al. (2011) that the X-rays lag the optical flux variations by $1.0 \pm 1.0$ days during the period 2008-2009.

A correlation analysis between the $\gamma$-ray light curve from 2008 August 5 to 2011 October 21 and $1 \mathrm{~mm}$ light curve from
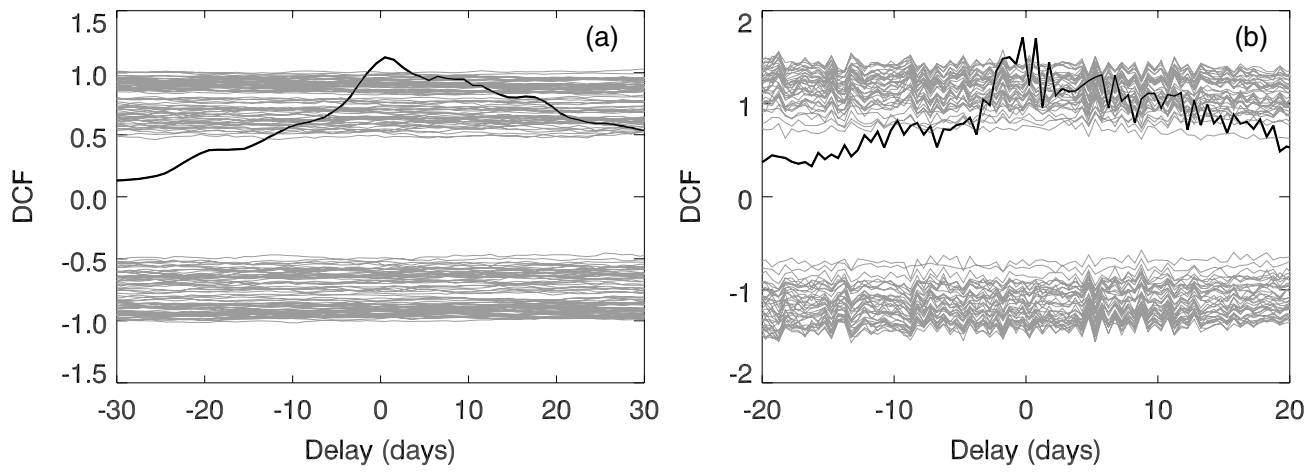

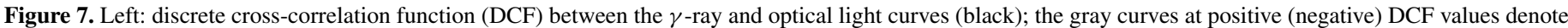

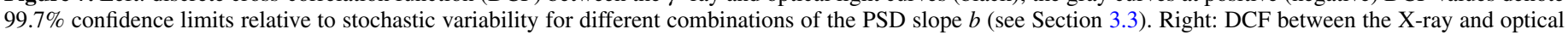
light curves (black). Negative delay means that high-energy flux variations lead those at optical wavelengths. 
2008 January 12 to 2011 October 27 was performed by Wehrle et al. (2012). These authors found a significant correlation between variations at the two wavelengths for delays from -1.5 to +3.5 days, which suggests that mm-wave variations are either simultaneous with $\gamma$-ray variations or slightly precede the latter.

Our analysis of the multi-frequency light curves therefore reveals a strong similarity in the general structure of contemporaneous $\gamma$-ray, X-ray, optical, and mm-wave outbursts and a statistically significant correlation between variations at different wavelengths. This suggests that:

1. The same ensemble of relativistic electrons that produces variable synchrotron optical emission participates in the production of variable $\gamma$-ray emission. This follows from the lack of significant time delays between the variations at these two wavebands. In fact, for values of the magnetic field typically inferred in the flaring regions of jets, $\sim 0.1-1 \mathrm{G}$, essentially the same energies of electrons are involved in both optical and $\gamma$-ray emission.

2. Given the above similarity in electron energies, the existence of orphan optical flares implies that either the density of seed photons changes with time, or changes in the strength and/or direction of the magnetic field cause such flares.

3. The emission regions at all four wavelengths are at least partially co-spatial, with the $\gamma$-ray emission exhibiting the fastest variability, $\tau_{\gamma}^{\min } \approx 1 / 5 \tau_{\mathrm{opt}}^{\min }$, while $\tau_{\mathrm{opt}}^{\min } \approx \tau_{\mathrm{X}}^{\min }$ and $\tau_{\mathrm{mm}}^{\min } \approx 30 \tau_{\mathrm{opt}}^{\min }$. Either the $\gamma$-ray flux is more sensitive to changes in the physical parameters than is the optical/X-ray flux, or the $\gamma$-ray emitting plasma fills $\sim 1 / 5$ of the optical/ $\mathrm{X}$-ray emission region. The similar X-ray and optical timescales of variability suggest that the corresponding emission regions are fully co-spatial. Although the mmwave emission region is $\sim 100$ times larger than that at $\gamma$-rays, a strict correspondence between the global maxima of the $\gamma$-ray and mm-wave outbursts implies that the $\mathrm{mm}$-wave region has sub-structures of different sizes, e.g., $\sim 0.01 \mathrm{pc}$ (the size of a turbulent cell within which the magnetic field is considered to be uniform; Wehrle et al. 2012), $\leqslant 0.4 \mathrm{pc}$ (the size of the mm-wave core; see Section 4 ), and $\sim 1 \mathrm{pc}$ (the size of a superluminal knot), with the most compact and variable features co-spatial with the $\gamma$-ray emission region.

4. The delay of X-ray with respect to optical flux variations, plus the similarity of the timescales of variability, imply a delay in the arrival of seed photons before they are scattered to X-ray energies. This favors a synchrotron origin of the seed photons from a location near to, but not coincident with, the scattering electrons. Such a situation can occur in the synchrotron self-Compton (SSC) mechanism for X-ray production, since there is a light-travel delay of synchrotron seed photons from the flare as they cross the flaring region if the angle to the line of sight is close to zero (Sokolov et al. 2004), as in 3C 454.3 ( $\Theta_{\circ} \sim 0.3-1.3$; see Section 4).

5. The dramatic difference in the amplitudes of the $\gamma$-ray and optical outbursts, the presence of orphan flares, and the tight correlation between $\gamma$-ray and optical variations without a significant delay favor the process of scattering of external photons by relativistic electrons that produce synchrotron optical emission up to $\gamma$-rays (the external Compton mechanism, EC) as the main mechanism for production of the $\gamma$-ray emission during the outbursts. However, a contribution from SSC cannot be avoided, since synchrotron seed photons are also produced by the relativistic electrons involved in the $\gamma$-ray production.

\section{BEHAVIOR OF THE PARSEC SCALE JET}

Figures 8 and 9 present sequences of $43 \mathrm{GHz}$ total and polarized intensity VLBA images of 3C 454.3. We use these sequences to follow changes in the flux density and polarization of the core, as well as the appearance, motion, and evolution of new features in the jet. The total intensity images are modeled by components with circular Gaussian brightness distributions in the same manner as described in Jorstad et al. (2005). At each epoch we identify the core, $A 0$, as the stationary feature located at the eastern end of the jet. The average angular size of the core in the model fits is $\left\langle a_{\text {core }}\right\rangle=0.05 \pm 0.02$ mas $(\sim 0.4 \mathrm{pc})$. Parameters of components include flux density $S$ (in Jy), separation $r$ (in mas), and position angle $\Theta$ (in deg) relative to the centroid of the core, and FWHM size $a$ (in mas). We compute the degree $P$ and position angle $\chi$ of linear polarization of components using an IDL program that calculates the mean values of the pixels at the position of each total intensity component and within an area equal to that of the size established by the modeling. All parameters of components are used to identify features across epochs in order to analyze the evolution of the jet. We define the inner direction of the jet $\Theta_{\text {jet }}$ equal to the value of $\Theta$ of the brightest knot within $0.1-0.3$ mas of the core. Figure 10 plots $\Theta_{\text {jet }}$, as well as the value of the average direction of the inner jet $\left\langle\Theta_{\text {jet }}\right\rangle=-92 \pm 20 \mathrm{deg}$, versus time. We find that the inner jet is oriented in the same direction as observed in 2004-2008, $\left\langle\Theta_{\text {jet }}\right\rangle=-95 \pm 8 \mathrm{deg}$ (Jorstad et al. $2010)$, although the standard deviation of $\left\langle\Theta_{\text {jet }}\right\rangle$ indicates that the jet executed greater swings during 2009-2011 than reported previously. This is likely the result of a smaller viewing angle of the jet during 2009-2011, which amplified changes in the angle of the jet as projected on the sky plane.

\subsection{Kinematics of the Parsec Scale Jet}

Figure 11 shows the results of modeling of the total intensity images within 1 mas of the core. We have identified features that can be associated with moving knots $K 1, K 2$, and $K 3$, as well as quasi-stationary feature $C$ identified in Jorstad et al. (2010). In comparison with the results reported in Jorstad et al. (2010), knot $K 1$ appears to have accelerated by a factor of $\sim 2$, although at a number of epochs the knot is confused with either $K 2$ or $C$. Knot $K 2$ moves with the same slow apparent speed, $\sim 3 c$, as seen during the later epochs analyzed in Jorstad et al. (2010). Although knot $K 3$ has faded dramatically $\left(S_{7 \mathrm{~mm}} \sim 0.3 \mathrm{Jy}\right)$, it has the same speed $\sim 4 c$ as reported previously. After the appearance of the new, very bright feature $K 09$ at the end of 2009 , knots $K 2$ and $K 3$ became too weak to be detected with a dynamic range of $\sim 1500: 1$. Knot $K 09$ was as bright as the core in the beginning of 2010 and even dominated the flux of the parsec scale jet in 2010 Summer. According to the modeling, knot $K 10$ appeared to be ejected at the end of 2010.

The apparent motions of knots $K 09$ and $K 10$ are complex, as seen in Figure 11. Knot $K 09$ appears to decelerate at a distance of $\sim 0.15$ mas from the core and then accelerate at a distance of $\sim 0.2$ mas. However, this is almost surely an artifact of the blending of $K 10$ with the core, which shifts the apparent centroid of the core downstream for some time, after which the separation of $K 09$ from the core rejoins the line representing a ballistic trajectory. Knot $K 10$ appears in the jet at $\Theta \approx-136^{\circ}$, south of the usual jet direction (Figure 9), then its trajectory curves 


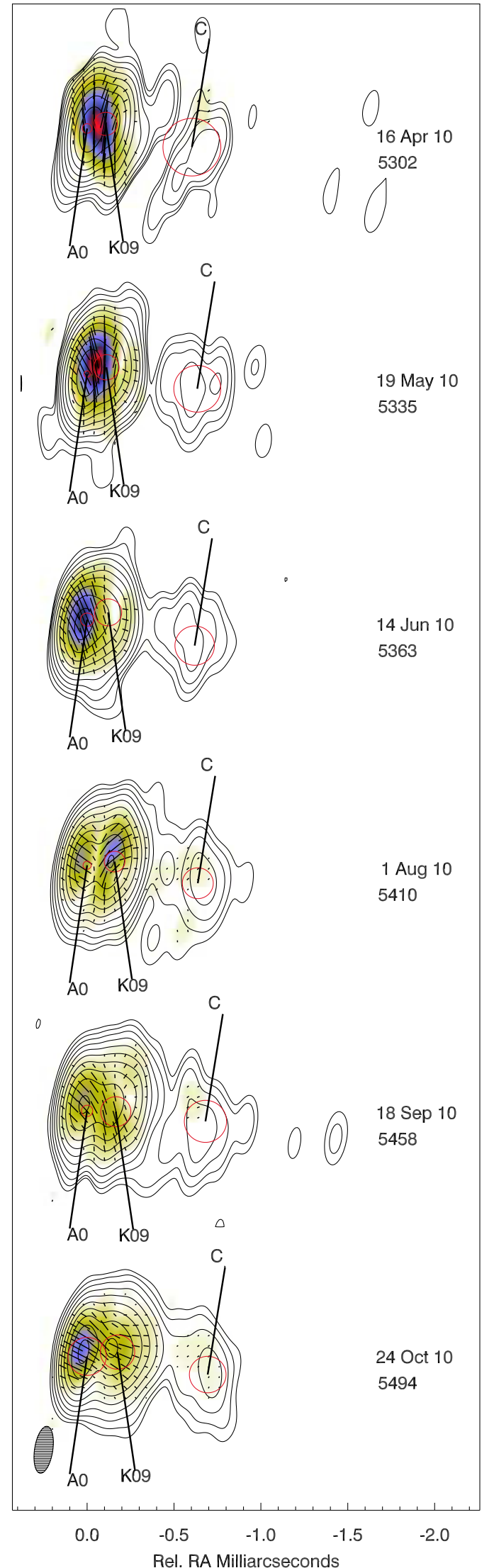

Figure 8. $43 \mathrm{GHz}$ total (contours) and polarized (color scale) intensity images of 3C 454.3 with $I_{\text {peak }}=19.80 \mathrm{Jy} \mathrm{beam}^{-1}, I_{\text {peak }}^{\text {pol }}=0.80 \mathrm{Jy} \mathrm{beam}^{-1}$, and a Gaussian restoring beam $=0.14 \times 0.33 \mathrm{mas}^{2}$ at P.A. $=-10^{\circ}$; contours represent $0.1 \%, 0.2 \%, \ldots, 51.2 \%, 99.5 \%$ of the peak intensity; line segments within the image show direction of linear polarization; red circles indicate position and size (FWHM) of components according to model fits.

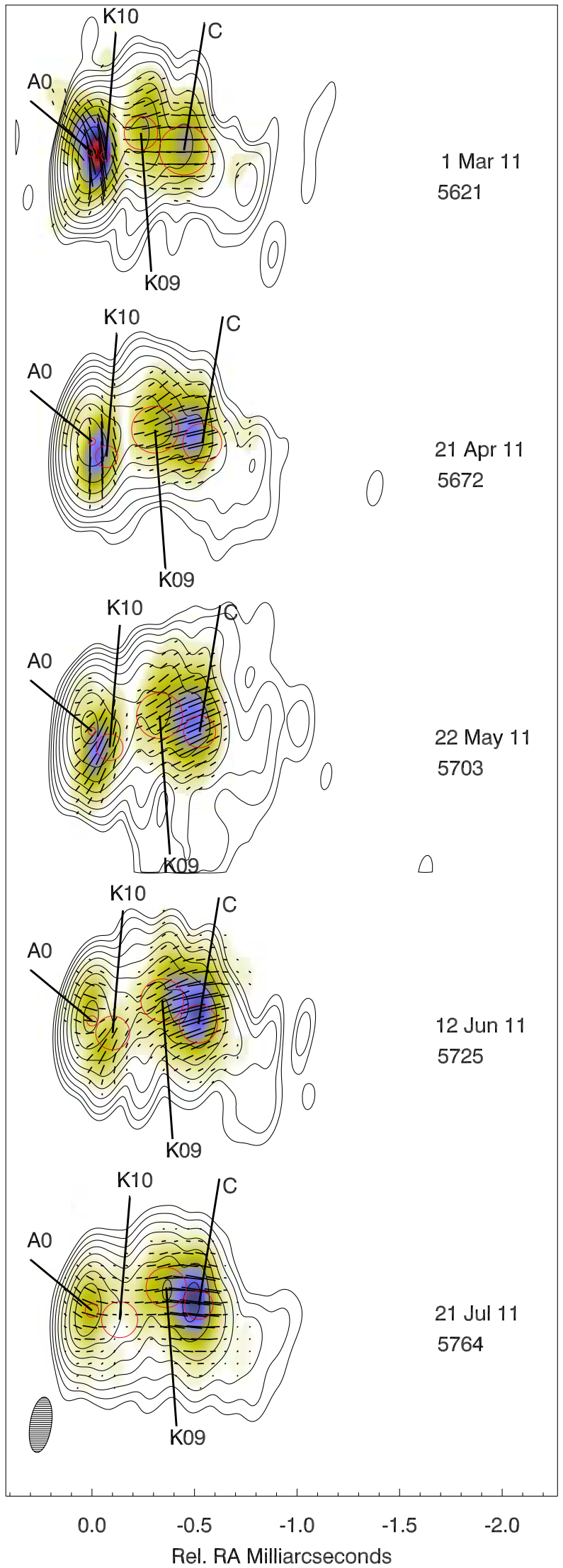

Figure 9. $43 \mathrm{GHz}$ total (contours) and polarized (color scale) intensity images of 3C 454.3 with $I_{\text {peak }}=16.29 \mathrm{Jy} \mathrm{beam}^{-1}, I_{\text {peak }}^{\text {pol }}=0.46 \mathrm{Jy}_{\text {beam }}{ }^{-1}$, and a Gaussian restoring beam $=0.14 \times 0.33$ mas $^{2}$ at P.A. $=-10^{\circ}$; contours represent $0.1 \%, 0.2 \%, \ldots, 51.2 \%, 99.5 \%$ of the peak intensity; line segments within the image show direction of linear polarization; red circles indicate position and size (FWHM) of components according to model fits. 


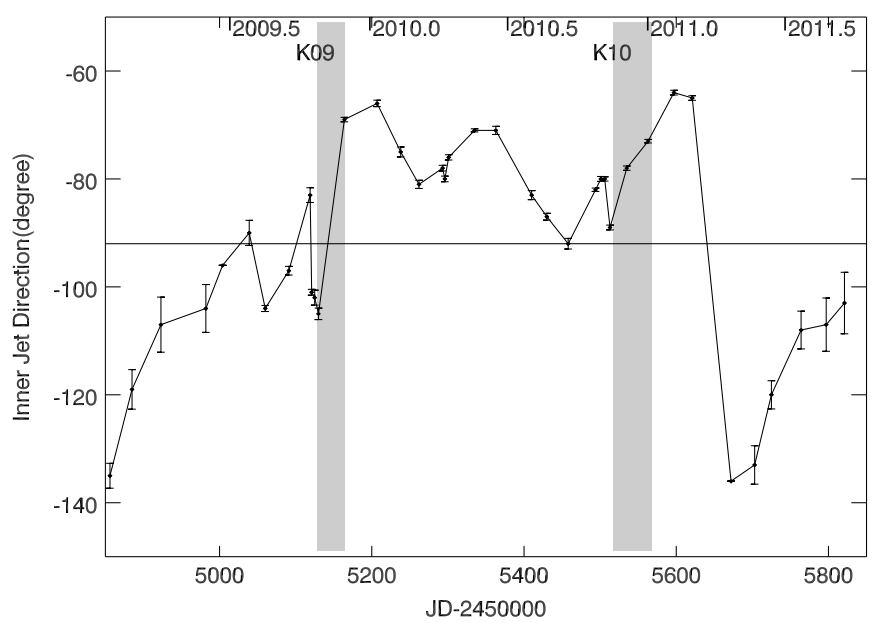

Figure 10. The inner jet (within 0.2 mas of the core) direction vs. time Horizontal solid line indicates the average direction of the jet, $-92 \pm 20 \mathrm{deg}$. The vertical gray areas mark times of passage of knots $K 09$ and $K 10$ through the VLBI core, as derived from the kinematics of the knots.

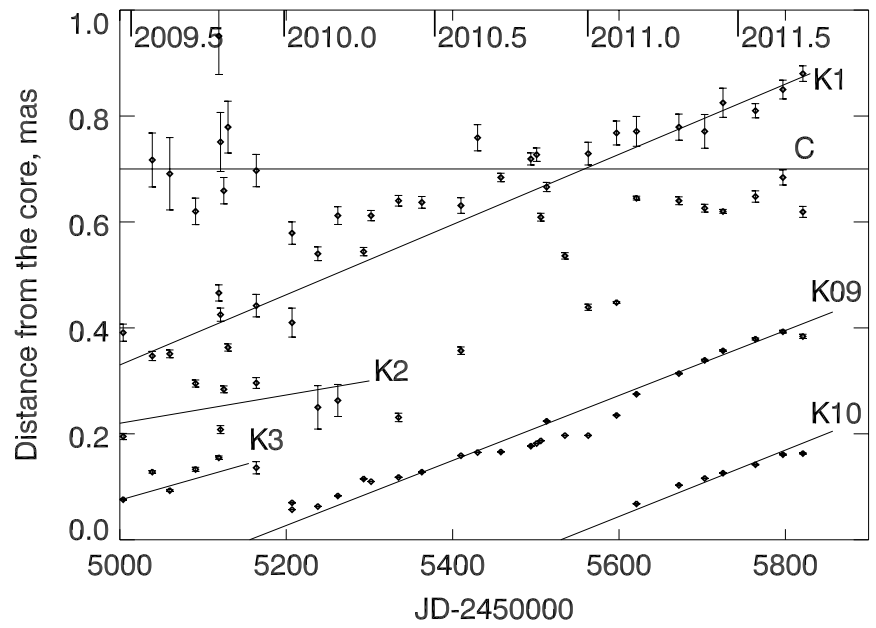

Figure 11. Distance of components from the core within 1 mas of the core based on the model fitting.

into the direction of the average projected jet axis, $\Theta_{\text {jet }} \approx-92^{\circ}$ (Figure 12). Using the technique developed in Jorstad et al. (2005), we have calculated for $K 09$ and $K 10$ the apparent speed, $\beta_{\text {app }}$, acceleration along and perpendicular to the jet, $\dot{\mu}_{\|}$and $\dot{\mu}_{\perp}$, time of ejection $T_{\circ},{ }^{26}$ timescale of flux variability, $\tau_{\mathrm{var}}$, Doppler factor, $\delta$, Lorentz factor, $\Gamma$, and viewing angle, $\Theta_{\circ}$. The values of these parameters are given in Table 5. According to Table 5, components $K 09$ and $K 10$ have similar apparent speeds, $\sim 9 c$, a value that falls within the range of $\beta_{\text {app }}$ observed previously in 3C 454.3 (Jorstad et al. 2001, 2010; Kellermann et al. 2004; Lister et al. 2009). Both $K 09$ and $K 10$ execute an acceleration perpendicular to the jet, which can be related to the apparent change of position angle near the core. Figure 12 suggests that the knots were ejected along different position angles, $K 09$ to the north and $K 10$ to the south with respect to the average jet axis. K09 also increases its proper motion along the jet, which can be attributed to an intrinsic acceleration (Homan et al. 2009). The values of the parameters $\delta, \Gamma_{\mathrm{b}}$, and $\Theta_{\circ}$ of $K 09$ are very close to those derived by Jorstad et al. (2005) from the kinematics of the jet in 1998-2001, while the Doppler factor

$26 T_{\circ}$ is the extrapolated time of coincidence of the centroid of a moving knot with the centroid of the core on the VLBA images.

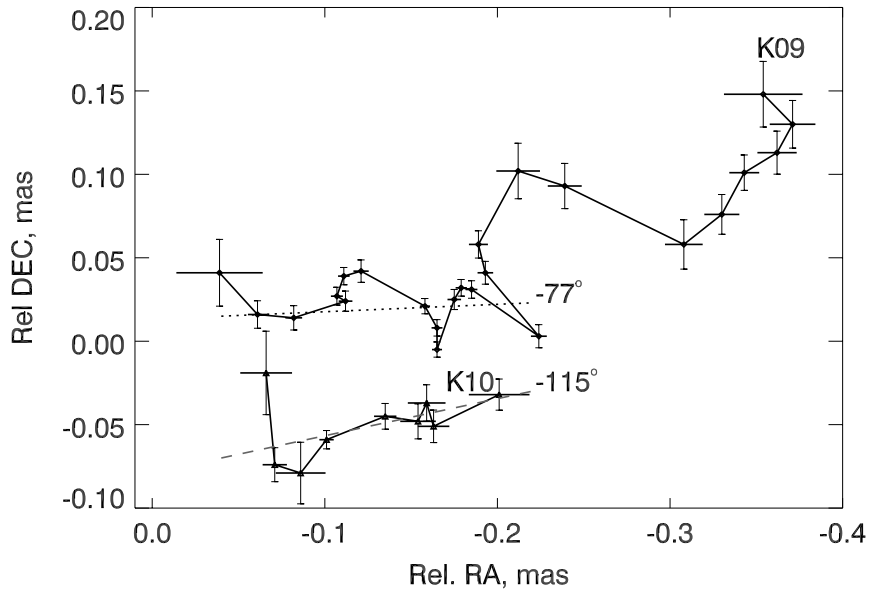

Figure 12. Trajectories of knots $K 09$ (diamonds) and $K 10$ (triangles); the dotted and dashed lines show the average position angle of $K 09$ and $K 10$, respectively, within 0.2 mas of the core.

Table 5

Parameters of Knots K09 and K10

\begin{tabular}{lcc}
\hline \hline Parameter & $K 09$ & $K 10$ \\
\hline$\mu\left(\mathrm{mas} \mathrm{yr}^{-1}\right)$ & $0.21 \pm 0.02$ & $0.19 \pm 0.03$ \\
$\dot{\mu}_{\|}\left(\mathrm{mas} \mathrm{yr}^{-2}\right)$ & $0.10 \pm 0.01$ & $\ldots$ \\
$\dot{\mu}_{\perp}\left(\mathrm{mas} \mathrm{yr}^{-2}\right)$ & $0.13 \pm 0.02$ & $1.10 \pm 0.22$ \\
$\beta_{\text {app }}(c)$ & $9.6 \pm 0.6$ & $8.9 \pm 1.7$ \\
$T_{\circ}$, yr & $2009.86 \pm 0.05$ & $2010.95 \pm 0.07$ \\
$T_{\circ}(\mathrm{RJD})$ & $5146 \pm 18$ & $5543 \pm 25$ \\
$S_{\max }(\mathrm{Jy})$ & $17.00 \pm 0.45$ & $7.10 \pm 0.16$ \\
$\tau_{\mathrm{var}}(\mathrm{yr})$ & $0.67 \pm 0.06$ & $0.24 \pm 0.02$ \\
$a(\operatorname{mas})$ & $0.12 \pm 0.02$ & $0.08 \pm 0.01$ \\
$\delta$ & $27 \pm 3$ & $51 \pm 4$ \\
$\Gamma_{\mathrm{b}}$ & $15 \pm 2$ & $26 \pm 3$ \\
$\Theta_{\circ}($ deg $)$ & $1.35 \pm 0.2$ & $0.4 \pm 0.1$ \\
$N$ & 24 & 7 \\
\hline
\end{tabular}

Notes. $\mu$ : proper motion; $\dot{\mu}_{\|}$: angular acceleration along the jet; $\dot{\mu}_{\perp}$ : angular acceleration perpendicular to the jet; $\beta_{\mathrm{app}}$ : apparent speed; $T_{\circ}$ : time of ejection; $S_{\max }$ : maximum flux; $\tau_{\mathrm{var}}$ : timescale of flux variability; $a$ : angular size of component at epoch of maximum flux; $\delta$ : Doppler factor, $\Gamma_{\mathrm{b}}$ : Lorentz factor; $\Theta_{\circ}$ : angle between velocity of component and line of sight; $N$ : number of epochs at which component was detected.

of $K 10$ is extreme, $\delta \sim 50$. The latter yields a much smaller viewing angle for $K 10$ with respect to $K 09$, in agreement with the different projected trajectories of the knots, which differ by $\sim 38^{\circ}$ (Figure 12). According to Table 5, the main difference in the derived values of $\delta$ results from the timescale of variability. $K 10$ fades faster than $K 09$ by a factor of 2.5 .

\subsection{Flux and Polarization Variability}

Figure 13 displays the overall $1 \mathrm{~mm}$ and $7 \mathrm{~mm}$ light curves of individual components in the inner jet. The total $7 \mathrm{~mm}$ flux is calculated as the sum of $A 0, K 09, K 10$, and $C$, depending on which feature is present at a given epoch according to the modeling of the images. The light curve of the core follows a smooth version of the variations at $1 \mathrm{~mm}$, although the contribution of other jet components to the $1 \mathrm{~mm}$ flux is significant, since in general the flux at $1 \mathrm{~mm}$ exceeds the core flux at $7 \mathrm{~mm}$ throughout the majority of epochs. Comparison of the $1 \mathrm{~mm}$ and inner jet light curves shows that during RJD: 5100-5200 and RJD: 5500-5550 the flux at $1 \mathrm{~mm}$ is higher than that at $7 \mathrm{~mm}$ from the inner jet, and the opposite is observed 


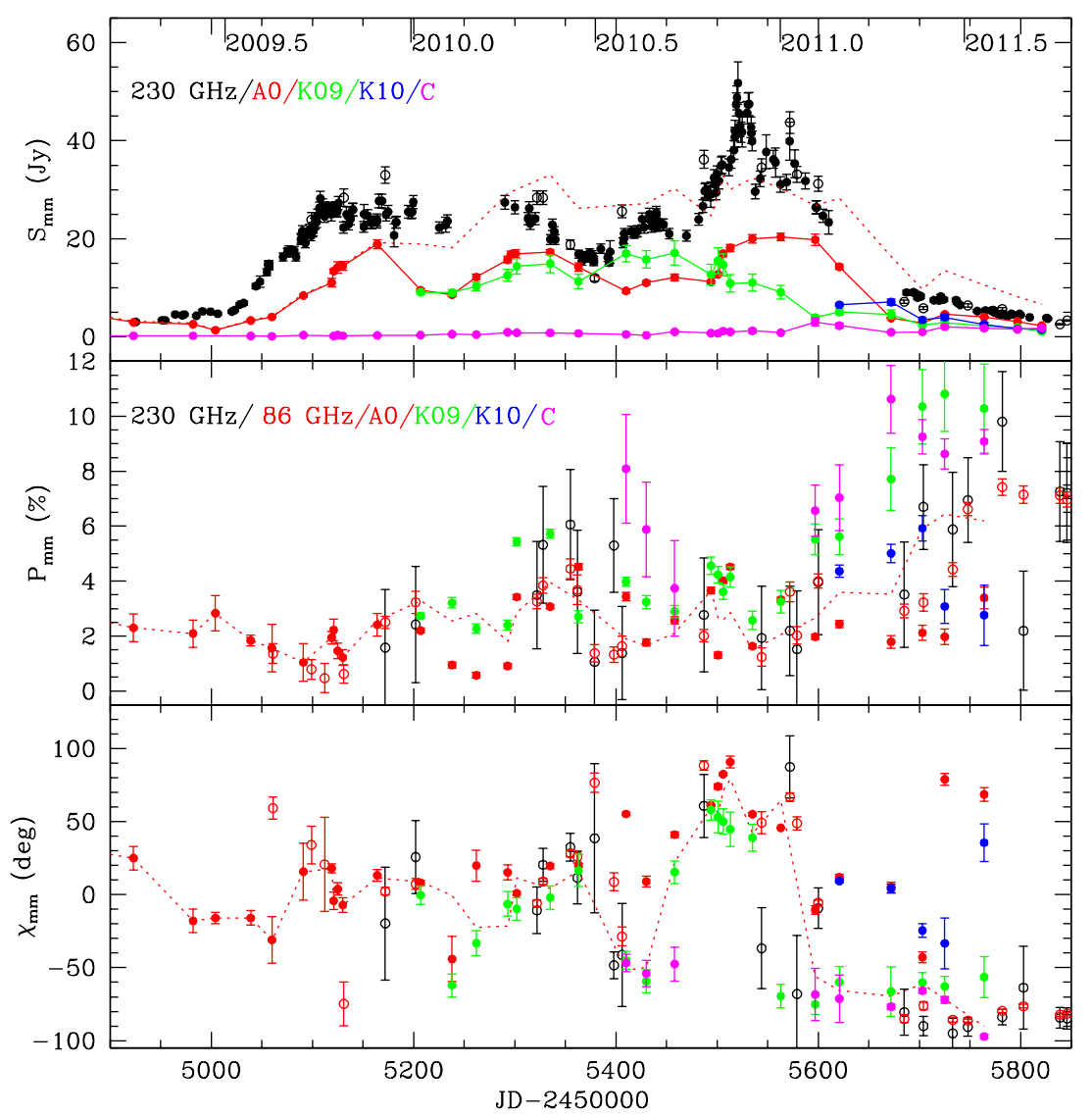

Figure 13. Total flux, $S$, degree, $P$, and position angle, $\chi$, of linear polarization at mm wavelengths vs. time. Top panel: the light curve at $230 \mathrm{GHz}$ obtained with the SMA (black filled cycles) and IRAM (black open circles) plus the light curves of the jet features: VLBI core, A0—red circles connected by the red solid line, stationary knot $C$-magenta circles and magenta line, knot $K 09$ - green circles and green line, and knot $K 10$-blue circles and blue line; the red dotted line shows the summed flux of all four jet knots $(A 0+C+K 09+K 10)$. Middle panel: degree of polarization from the whole source at $230 \mathrm{GHz}$ (black open circles) and $86 \mathrm{GHz}$ (red open circles); $P$ of the jet features at $43 \mathrm{GHz}$ : the core $A 0$ (red filled circles), knot $C$ (magenta filled circles; $P$ of knot $C$ is divided by a factor of three to display alongside other features), $K 09$ (green filled circles), and $K 10$ (blue filled circles); the red dotted line shows the summed $P$ of all four jet knots. Bottom panel: position angle of polarization from the whole source at $230 \mathrm{GHz}$ and $86 \mathrm{GHz}, \chi$ of the jet features at $43 \mathrm{GHz}: A 0, C, K 09$, and $K 10$ (designations are the same as in the middle panel); the red dotted line shows the summed $\chi$ of all four jet knots.

within RJD: 5300-5500 and after RJD: 5600. The bright $1 \mathrm{~mm}$ states, relative to $7 \mathrm{~mm}$, are modeled to be contemporaneous with the times when knots $K 09$ and $K 10$ were passing through the core. The lower $7 \mathrm{~mm}$ flux can be explained by a temporary suppression of the $7 \mathrm{~mm}$ flux outburst resulting from opacity increases in the $7 \mathrm{~mm}$ core as a superluminal knot moves through it. After a significant increase of the $1 \mathrm{~mm}$ flux in 2009 Autumn, the $1 \mathrm{~mm}$ flux remains at a high level for more than a year, a circumstance that we associate with the appearance in the jet of the very bright knot $K 09$. Although the flux of the core decreased significantly after the ejection of $K 09$, the core was still brighter than during quiescent states in 2009 Spring and 2011 Summer when there is good agreement between the $1 \mathrm{~mm}$ and $7 \mathrm{~mm}$ core light curves. This implies that the contribution of the jet outside 1 mas to the emission at $1 \mathrm{~mm}$ is negligible.

Figure 13 displays the degree of linear polarization $P$ versus time at $\mathrm{mm}$ wavelengths. The polarization from the whole source at 1 and $3 \mathrm{~mm}$ changes from $0 \%$ to $\sim 10 \%$ and agrees very well with that of the inner jet (dashed line). This presents another argument in favor of the emission at 1-3 mm arising mostly from the inner jet that includes the core and components within 1 mas of the core. The polarization of the core ranges from $1 \%$ to $\sim 5 \%$, with a modest increase of $P$ during the outbursts. The polarization of moving knots $K 09$ and $K 10$ lies within 2\%-6\%; however, as $K 09$ approaches stationary feature $C, P$ increases significantly for both knots and reaches $12 \%$ and $30 \%$ for $K 09$ and $C$, respectively (see Figure 9). This supports the idea that the knots experience an interaction with the ambient jet, most likely in the form of a shock, since the position angle of polarization of both knots aligns with the jet direction. This is a primary signature of a transversely oriented shock, with the magnetic field compressed along the shock front (Hughes et al. 1985, 1989). At this time, knots $K 09$ and $C$ appear to contribute significantly to the polarized emission at 1 and $3 \mathrm{~mm}$ and $P_{1 \mathrm{~mm}}$ rises up to $10 \%$. Although the flux of $C$ increases slightly, the total flux density continues to fade at $\mathrm{mm}$ wavelengths along with the very long baseline interferometry (VLBI) core.

During outbursts I and III the polarization position angle $\chi_{\text {core }}$ of the core at $7 \mathrm{~mm}$ rotates from $+16^{\circ}$ to $-44^{\circ}$ and from $+91^{\circ}$ to $-11^{\circ}$, respectively (Figure 13 ). In addition, $\chi_{3 \mathrm{~mm}}$ rotates in a similar manner, although the range of rotation is greater than for $\chi_{\text {core }}$, especially during outburst I. Figures 8, 9, and 13 show that the polarization vectors of both $K 09$ and $K 10$ undergo rotations, as well. Figure 14 compares the evolution of $\chi_{K 09}$ and $\chi_{K 10}$ with distance from the core. Both knots appear in the jet with polarization vectors oriented perpendicular to the jet axis, but between separations of 0.05 and 0.18 mas the EVPAs of both knots swing, although the rotations are in the opposite direction at the same distance from the core. These rotations might be a signature of a large intrinsic Faraday rotation measure near the 


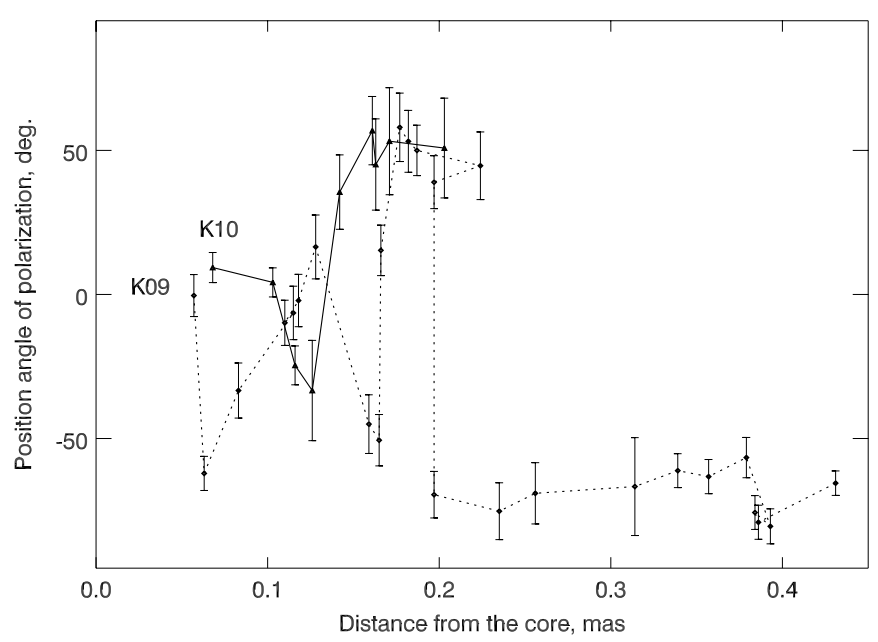

Figure 14. Position angle of polarization of knots $K 09$ (diamonds, dotted line) and $K 10$ (triangles, solid line) vs. their distance from the core.

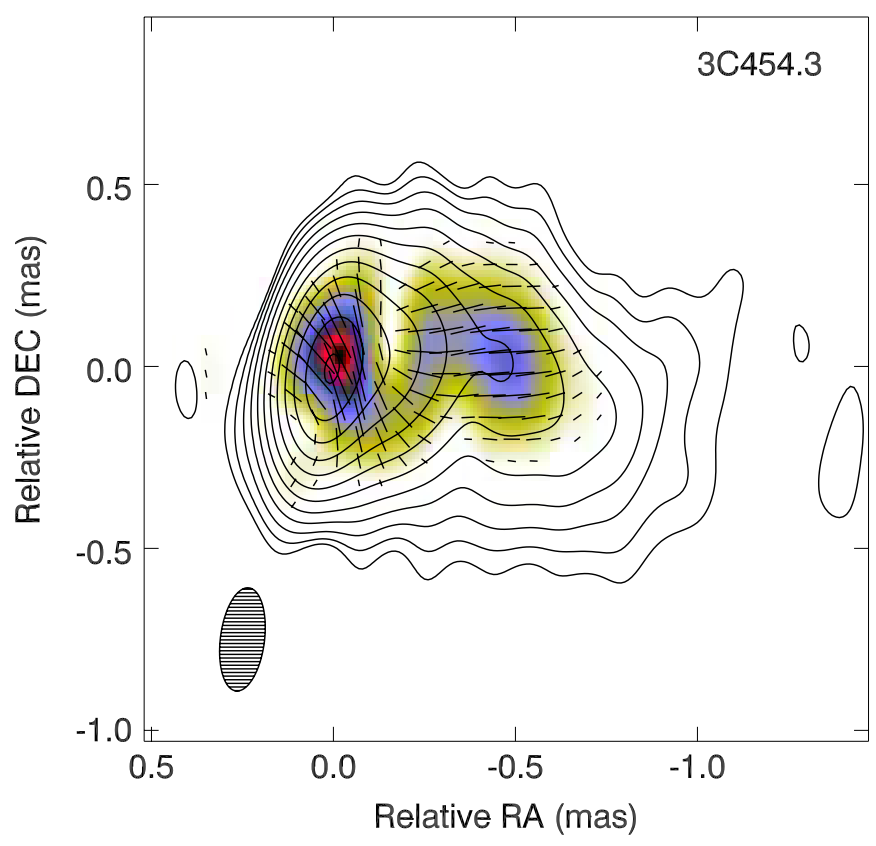

Figure 15. Composite total (contours) and polarized (color) intensity image of $3 \mathrm{C} 454.3$, which is the average of all 35 epochs of VLBA data at $43 \mathrm{GHz}$ from 2009 April to 2011 August, with $I_{\text {peak }}=11.51 \mathrm{Jy} \mathrm{beam}^{-1}$, $I_{\text {peak }}^{\text {pol }}=0.11 \mathrm{Jy} \mathrm{beam}^{-1}$, and beam $=0.14 \times 0.33$ mas $^{2}$ at P.A. $=-10^{\circ}$; contours represent $0.025 \%, 0.05 \%, \ldots, 25.6 \%, 51.2 \%$ of the peak intensity; line segments within the image show direction of polarization.

core caused by a toroidal structure of the magnetic field. In this case the different directions of rotation of $\chi_{K 09}$ and $\chi_{K 10}$ can be readily explained by a change in the sign of the magnetic field with respect to the line of sight, assuming that the knots are propagating along different sides of the jet, as can be inferred from the knot's trajectories (Figure 12 ). At $\sim 0.2$ mas from the core, $\chi_{K 09} \approx \chi_{K 10} \approx 50^{\circ}$, oblique to the jet direction, and at distances $>0.2$ mas $K 09$ has a stable EVPA, aligned with the jet axis, as well as with the EVPA of stationary knot $C$. Such a behavior is consistent with the knots being transversely oriented shocks propagating down the jet, which has a turbulent magnetic field (Hughes et al. 1985, 1989). Figure 15 shows the composite structure of the parsec-scale jet emission from 2009 April to 2011 August. The image is obtained by summing Stokes $I, Q, U$

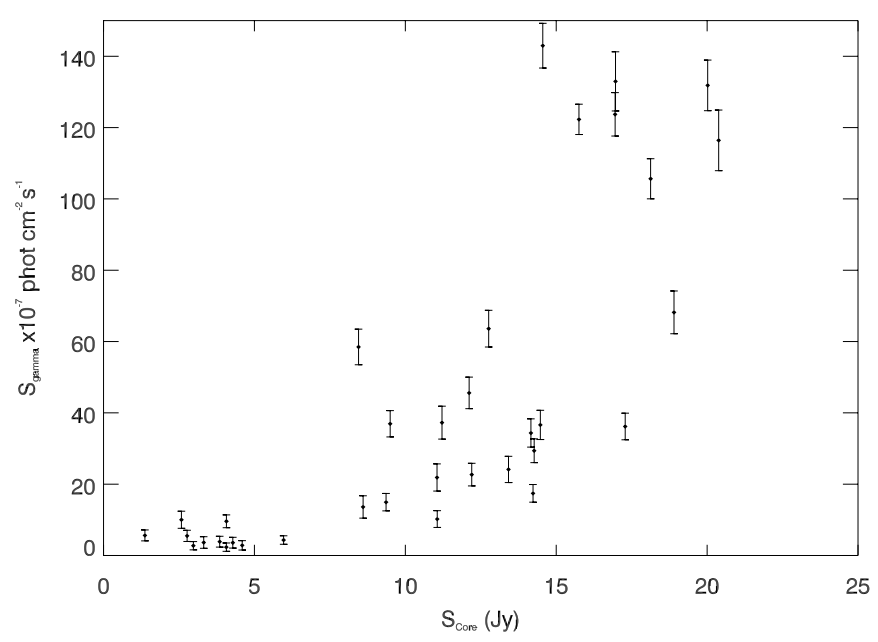

Figure 16. Gamma-ray flux density vs. flux density at $43 \mathrm{GHz}$ in the VLBI core for simultaneous measurements.

parameter maps over all 35 epochs obtained during this period (each map was convolved with the same restoring beam). The image represents an active state of the inner jet, since the $I_{\text {peak }}$ is a factor of $\sim 10$ brighter than during a quiescent state. A spiraltype structure is apparent in polarized intensity up to $\sim 0.4$ mas from the core, with a different direction of the EVPAs on the southwest and northwest edges of the polarized emission region. Farther down the jet, the position angle of polarization aligns with the jet direction, as expected if a turbulent magnetic field becomes partially ordered along the front of a transverse shock. This region of the jet is dominated by the contribution from knots $K 09$ and $C$. This picture is consistent with the scenario, proposed by Jorstad et al. (2007), that the mm-wave core is located at the end of the acceleration zone, where the jet energy density is dominated by the Poynting flux of a toroidal magnetic field. Near the core the flow becomes kinetic energy dominated, with the magnetic field becoming turbulent.

\subsection{Connection between Jet Activity and the Gamma-Ray Outbursts}

We analyze the relation between the $\gamma$-ray flux, $S_{\gamma}$, and the $43 \mathrm{GHz}$ flux density of the VLBI core, $S_{\text {core }}$. Figure 16 shows $S_{\gamma}$ versus $S_{\text {core }}$ for all VLBA epochs (35), with the $\gamma$-ray photons integrated over the $24 \mathrm{hr}$ centered on the VLBA observation. There is a strong correlation between variations at $\gamma$-rays and in the core. The linear Pearson correlation coefficient, $r=0.77$, is statistically significant at the $99.9 \%$ confidence level. The flux density of the core increases by a factor of 16 while the $\gamma$-ray flux rises by a factor of $\sim 65$. This implies that the $\gamma$-ray flux is relativistically beamed in the same manner or more strongly than the radio flux, as suggested previously by Jorstad et al. (2001) and Kovalev et al. (2009). The relation between $S_{\gamma}$ and $S_{\text {core }}$ does not fit a simple linear dependence, rather, two relationships can be inferred: (1) for $S_{\text {core }} \lesssim 14 \mathrm{Jy}$ the dependence is almost linear, and (2) for $S_{\text {core }} \gtrsim 14$ Jy the dependence is roughly quadratic. In addition, Wehrle et al. (2012) have found a statistically significant correlation between the $\gamma$-ray and $1 \mathrm{~mm}$ light curves, with no lag.

Enhanced flux density of the core region in a VLBI image of a blazar usually corresponds to the emergence of a new disturbance into the flow in the radio-emitting zone of the jet (e.g., Savolainen et al. 2002). Comparison of Tables 1 and 5 indicates that knots $K 09$ and $K 10$ passed through the mm-wave 
VLBI core close to the time of the $\gamma$-ray peaks of outbursts I and III (within the $1 \sigma$ uncertainty of the ejection time of 18 and 25 days, respectively). The duration of these $\gamma$-ray outbursts is comparable to the time needed for a knot to go through the core, $90 \pm 15$ days, for the average core size of 0.05 mas and average proper motion of knots of 0.20 mas $\mathrm{yr}^{-1}$. In addition, during the $\gamma$-ray outbursts we observe an increase in the core opacity, as revealed by comparison of the $1 \mathrm{~mm}$ and $7 \mathrm{~mm}$ light curves, and rotation of the polarization vector in the core and at $3 \mathrm{~mm}$. These trends argue in favor of the $\gamma$-ray outbursts coinciding with the passage of superluminal knots through the core. Although we did not detect a superluminal knot associated with $\gamma$-ray outburst II, Figure 13 shows that both the core and K09 underwent flares during outburst II (RJD: 5275-5349), with the flux reaching $S \sim 18 \mathrm{Jy}$. Moreover, we observed an increase of the degree of polarization in the core and at $1 \mathrm{~mm}$ and $3 \mathrm{~mm}$, along with a rotation of $\chi$ at 1 and $3 \mathrm{~mm}$. Based on the history of $3 \mathrm{C} 454.3$, it is likely that this increase in the flux and polarization of the core during the $\gamma$-ray event was caused by the passage of a new superluminal knot through the core, with a flux of $\leqslant 7$ Jy. Summer-Autumn 2010 would have been the best period to detect this hypothetical new component in the jet. However, during this interval $K 09$ was still close to the core, between 0.12 mas and 0.20 mas (Figure 11). Although $K 09$ is distinct from the core at the high resolution of our observations (see Figure 8), the resolution is insufficient to identify a weaker knot situated between the core and an extremely bright knot within 0.20 mas. The increase in the flux of $K 09$ along with the core during $\gamma$-ray outburst II might be an artifact of modeling with circular Gaussians of such complex structure, but $K 09$ continued to be brighter than the core as the core faded in mid-2010 (Figure 13). This suggests that K09 might have been blended with the putative superluminal knot. The subsequent dramatic increase of the flux in the core in 2010 November and ejection of K10 further reduced the possibility of detecting any propagating disturbance associated with $\gamma$-ray outburst II. Overall, comparison of the $\gamma$-ray and mm-wave behavior provides strong evidence of a tight connection between enhanced $\gamma$-ray emission and the passage of superluminal knots through the mm-wave core located at a distance $\sim 15-20$ pc from the central engine (Jorstad et al. 2010; Pushkarev et al. 2012).

\section{SPECTRAL BEHAVIOR}

We analyze the spectral behavior of 3C 454.3 from $\gamma$-ray to mm wavelengths with emphasis on outbursts I, II, and III. This includes studies of (1) the $\gamma$-ray spectral index in the energy range of $0.1-200 \mathrm{GeV}$; (2) the X-ray spectral index at $0.3-10 \mathrm{keV}$; (3) optical spectra in the range of $4000-7550 \AA$ (181 spectra); and (4) spectral indices based on fluxes measured simultaneously (within 1 day) at different bands from $U V$ to far-IR, and at wavelengths from 1.3 to $8 \mathrm{~mm}$. Tables 7 and 8 indicate the number of simultaneous flux measurements at a given pair of wavelengths. We define the spectral index $\alpha$ such that the flux density $S_{v} \propto v^{-\alpha}$.

\subsection{Gamma-Ray, X-Ray, and Millimeter-wave Spectral Indices}

We have derived weekly $\gamma$-ray spectral indices $\alpha_{\gamma}$ between 0.1-200 GeV using a simple power-law model with variable photon index and normalization ("prefactor") to represent the quasar emission. Frozen spectral parameters (values from the 2FGL catalog; Nolan et al. 2012) were used for other sources

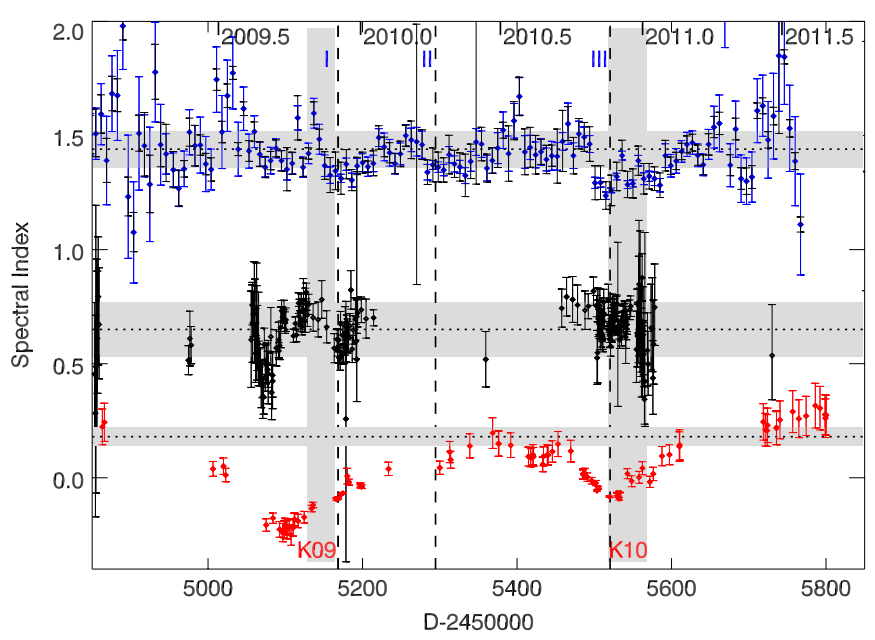

Figure 17. Gamma-ray at $0.1-200 \mathrm{GeV}$ (blue), X-ray at $0.3-10 \mathrm{keV}$ (black), and $\mathrm{mm}$-wave between 1 and $8 \mathrm{~mm}$ spectral indices vs. time; the dotted lines indicate the average spectral indices of $\alpha_{\gamma}$ and $\alpha_{\mathrm{X}}$, and $\alpha_{\mathrm{mm}}$ of a quiescent state, corresponding $1 \sigma$ uncertainties are marked by the horizontal gray areas; the vertical gray areas mark times of passage of knots $K 09$ and $K 10$ through the VLBI core; the dashed vertical lines mark peaks of the $\gamma$-ray emission during flares I, II, and III.

within $15^{\circ}$ radius of $3 \mathrm{C} 454.3$ to guide the maximum likelihood routine. Figure 17 shows the derived values of $\alpha_{\gamma}$, which varies from 1.2 to 1.8 with an average $\left\langle\alpha_{\gamma}\right\rangle=1.44$ and standard deviation $\sigma_{\gamma}=0.08$, which is less than the average uncertainty of an individual measurement $\sigma_{\gamma}^{1}=0.13$. However, the spectral indices averaged over a month-long interval centered on the peak of outburst are $1.33 \pm 0.03$ (flare I), $1.37 \pm 0.03$ (flare II), and $1.28 \pm 0.03$ (flare III). This implies a harder $\gamma$-ray spectrum during the highest states of $\gamma$-ray emission, a trend studied in detail by Ackermann et al. (2010) and Abdo et al. (2011).

We have derived 201 spectral indices, $\alpha_{\mathrm{X}}$, between 0.3 and $10 \mathrm{keV}$ from 2009 April 25 to 2011 August 1. The X-ray spectral index varies from 0.3 to 0.8 with an average value of $\left\langle\alpha_{\mathrm{X}}\right\rangle=0.65 \pm 0.12$, while the mean uncertainty of an individual measurement $\sigma_{\mathrm{X}}^{1}=0.10$ (Figure 17). This behavior is consistent with the conclusion of Raiteri et al. (2011) that the spectral variations are dominated by the noise of the measurement uncertainties. Indeed, during both outbursts for which X-ray data are available (I and III), $\alpha_{\mathrm{X}}$ exhibits random fluctuations around $\left\langle\alpha_{X}\right\rangle$. However, $\sim 3$ months before the peak of outburst I, the spectral index flattens over 19 consecutive measurements from RJD: 5065 to RJD: 5090, with an average value of $0.44 \pm$ 0.08 .

We use the data collected at the SMA, IRAM, and Metsähovi Radio Observatory facilities to derive the mm-wave spectral index $\left(\alpha_{\mathrm{mm}}\right)$ between 1.3 and $8 \mathrm{~mm}$. We have obtained 92 spectral indices from measurements simultaneous within 1 day. Figure 17 shows $\alpha_{\mathrm{mm}}$ versus epoch. The spectral index changes from +0.3 to -0.25 , very similar to the range observed in 2004-2008 (Jorstad et al. 2010). The figure also shows times of ejection of knots $K 09$ and $K 10$ from the core. Although $\alpha_{\mathrm{mm}}$ is slightly steeper during quiescent states than $\alpha_{\mathrm{mm}}^{\mathrm{q}}=$ $0.18 \pm 0.04$ measured in 2004-2008, the main feature of the $\alpha_{\mathrm{mm}}$ behavior - that the mm-wave spectral index reaches a local minimum just before the ejection of a superluminal knot-holds for both knots $K 09$ and $K 10$. This pattern was also observed for knots $K 1, K 2$, and $K 3$ (Jorstad et al. 2010). Unfortunately, there is a gap in the radio data during $\gamma$-ray outburst II that prevents us from using changes in $\alpha_{\mathrm{mm}}$ to search for signs of 


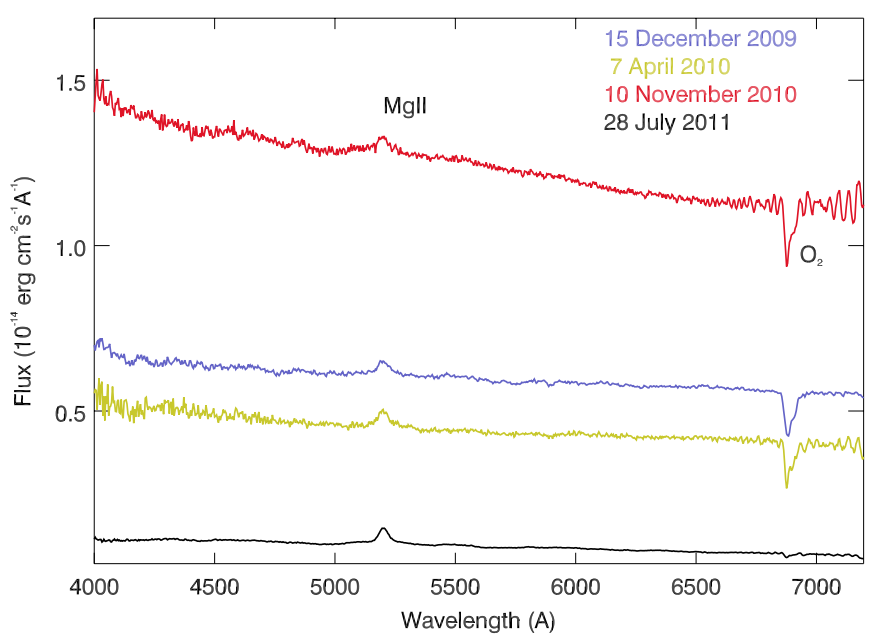

Figure 18. Optical spectrum of $3 \mathrm{C} 454.3$ in the observer's frame at four epochs.

the ejection of the hypothetical knot discussed in Section 4.3. During the events associated with knots $K 09$ and $K 10$, the spectrum becomes inverted with spectral indices of $-0.25 \pm$ $0.05\left(\alpha_{\mathrm{mm}}^{\mathrm{K} 09}\right)$ and $-0.08 \pm 0.01\left(\alpha_{\mathrm{mm}}^{\mathrm{K} 10}\right)$, implying an increase in opacity at $\mathrm{mm}$ wavelengths during the events. The fact that $\alpha_{\mathrm{mm}}^{\mathrm{K} 09}<\alpha_{\mathrm{mm}}^{\mathrm{K} 10}$ suggests a significant contribution of optically thin emission from $K 09$ to the total flux at mm-wavelengths during the $K 10$ event.

Figure 17 reveals similarity in variations of $\alpha_{\gamma}$ and $\alpha_{\mathrm{mm}}$. Both spectral indices flatten at/near the time of ejection of superluminal knots and steepen during quiescent periods (2009 Spring, 2010 Summer, and 2011 Spring). However, the similarity is not sufficient to claim a statistically significant correlation between the indices. Although the measurements of $\alpha_{X}$ are sparse and noisy, a flattening of $\alpha_{\mathrm{X}}$ at RJD: 5065-5090, which is significant at the $2 \sigma$ level, coincides with the period of the flattest $\alpha_{\mathrm{mm}}$ (RJD: 5075-5110) that precedes the ejection of $K 09$.

\subsection{Broad Emission Lines and Optical Continuum}

Figure 18 shows four spectra of the quasar at the epochs of the brightest (or next to brightest) $\gamma$-ray states during outbursts I, II, and III, and at a quiescent state in 2011 Summer. The most prominent emission features in the Steward Observatory spectra (2150-4060 $\AA$ in the rest frame) are broad $\operatorname{Mg}$ II $(\lambda=2800 \AA)$ and blended Fe II multiplets. A time series analysis between variations in the line and continuum emission is important for determining the size, geometry, and location of the broad emission line region (BLR). A number of studies have found that the size of BLR is proportional to the AGN luminosity, $R_{\mathrm{BLR}} \propto L_{5100}^{\kappa}$, where $\kappa$ varies from 0.5 to 0.7 (e.g., Kaspi et al. 2000 ), with $R_{\mathrm{BLR}}$ of low-luminosity AGNs falling in the range of 10-100 lt-day. This implies that the size of BLR in luminous quasars $\geqslant 1$ lt-yr. In general, analyses of emission line variations in blazars, including 3C 454.3, do not reveal a connection between line and continuum flux variations (3C 454.3: Raiteri et al. 2008, 1222+216: Smith et al. 2011, and 1633+384: Raiteri et al. 2012). However, a recent analysis by León-Tavares et al. (2013) of the optical spectra of 3C 454.3 obtained at Steward Observatory during 2008-2011 suggests the existence of significant variations in the $\mathrm{Mg}$ II line, corresponding to a flare-like event, during outburst III that challenges the standard model of the BLR in blazars. We possess the same data as analyzed by León-Tavares et al. (2013). Unfortunately, the data miss the 2-week period from 2010 November 16 to 30 during

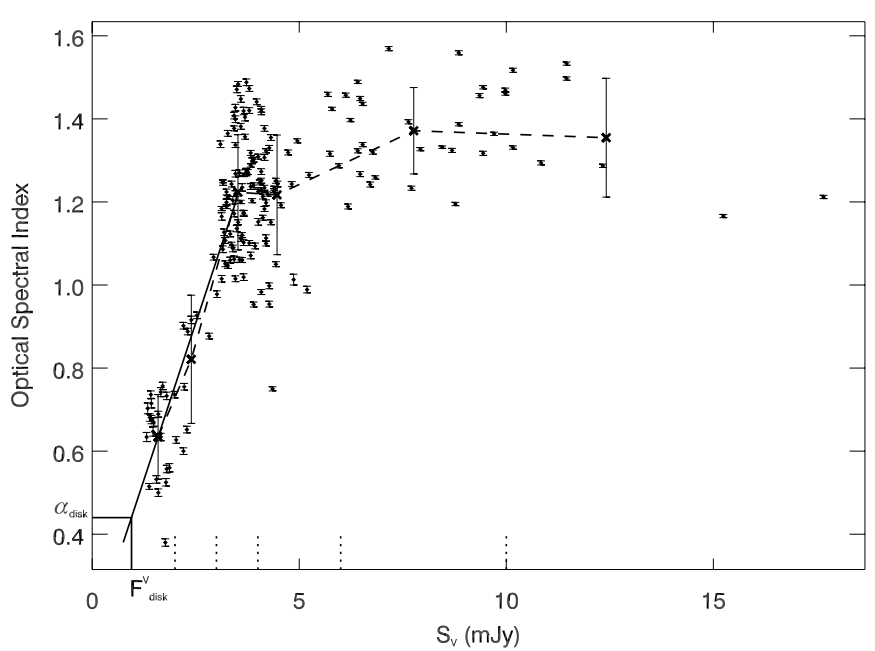

Figure 19. Spectral index vs. brightness of the optical continuum in $V$ band in the observer's frame; crosses show average values of $\alpha_{\text {cont }}$ within different intervals of flux, $S_{\mathrm{V}}$ (Table 6), connected by the dashed line; dotted vertical segments show intervals of the averaging; the solid line is a linear fit of the dependence for $S_{\mathrm{V}}<4.0 \mathrm{mJy}$.

Table 6

Spectral Index of Optical Continuum and Slope of $P(\lambda)$ Dependence

\begin{tabular}{lcccr}
\hline \hline $\begin{array}{l}\text { Interval of } S^{\mathrm{a}} \\
(\mathrm{mJy})\end{array}$ & $\alpha_{\text {cont }}$ & $N$ & $\begin{array}{c}B \times 10^{-5} \\
\left(\AA^{-1}\right)\end{array}$ & $N_{\mathrm{B}}$ \\
\hline $0-2.0$ & $0.63 \pm 0.10$ & 21 & $0.39 \pm 0.24$ & 35 \\
$2.0-3.0$ & $0.82 \pm 0.15$ & 10 & $0.75 \pm 0.42$ & 28 \\
$3.0-4.0$ & $1.22 \pm 0.14$ & 72 & $0.52 \pm 0.45$ & 109 \\
$4.0-6.0$ & $1.22 \pm 0.14$ & 43 & $0.33 \pm 0.33$ & 30 \\
$6.0-10.0$ & $1.37 \pm 0.10$ & 27 & $0.18 \pm 0.34$ & 32 \\
$>10.0$ & $1.35 \pm 0.14$ & 8 & $-0.23 \pm 0.31$ & 8 \\
\hline
\end{tabular}

Note. ${ }^{\text {a }} S$ is the flux in $V$ band for $\alpha_{\text {cont }}$ measurements and in $R$ band for slope $B$ measurements; $N, N_{\mathrm{B}}$ is the number of $\alpha_{\text {cont }}$ and slope $B$ measurements in each flux interval, respectively.

the brightest $\gamma$-ray and optical state in 3C 454.3. Since the Yale University blazar monitoring group obtained optical spectra of the quasar during this period (C. M. Urry 2013, private communication), we defer to an analysis of time variations of emission lines to the work by the Yale University blazar group.

We have corrected the spectra of 3C 454.3 for Galactic extinction, performed Gaussian fits to the emission features, subtracted the fits from each of 181 spectra, and approximated the residual continuum by a power law, $S_{v} \propto v^{-\alpha_{\text {cont }}}$, within the $\log _{10} v$ range from 14.65 to 14.85 ( $\lambda$ from 4235 to $6712 \AA$ ) to avoid the atmospheric $\mathrm{O}_{2}$ absorption feature and noisy edges of the spectra (the wavelength range is from 2278 to $3611 \AA$ in the quasar rest frame). Figure 19 plots $\alpha_{\text {cont }}$ versus flux in $V$ band from simultaneous measurements (within $\sim 1 \mathrm{hr}$ ). Table 6 lists average values of $\alpha_{\text {cont }}$ with their standard deviations for different flux intervals. The spectral index of the continuum steepens by a factor of two, from $\sim 0.6$ to $\sim 1.2$, as the flux increases from $\sim 1.5 \mathrm{mJy}$ to $3.5 \mathrm{mJy}$, while the scatter of $\alpha_{\text {cont }}$ values does not exceed 0.15 within each interval of averaging. A further increase of the flux up to $\sim 10 \mathrm{mJy}$ does not change the average spectral index significantly, although a slight steepening, up to 1.35 , toward higher flux is observed. Such behavior of $\alpha_{\text {cont }}$ is a direct indication that the continuum consists of at least two components, blue and red. The blue component dominates at low brightness states and can be attributed to big blue bump (BBB) emission produced by the accretion disk (Smith et al. 1988; Raiteri et al. 

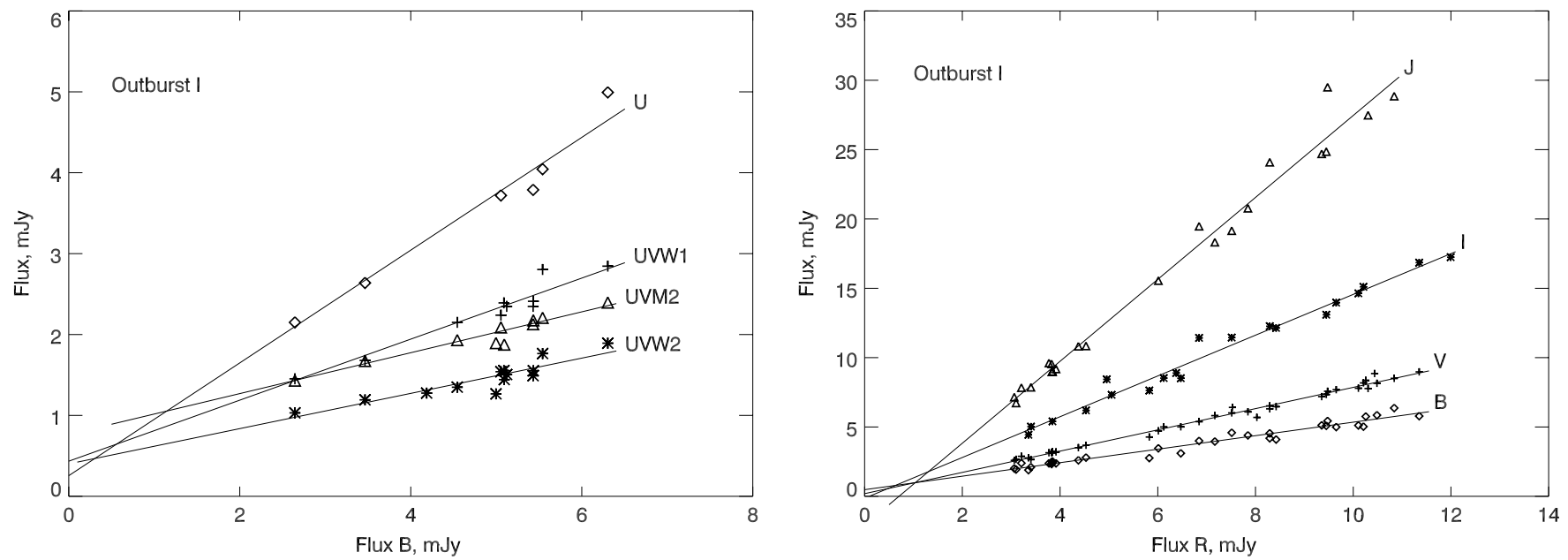

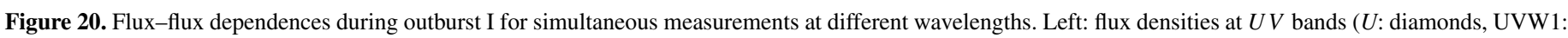

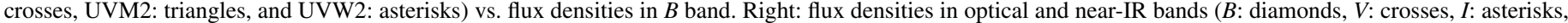
and $J$ : triangles) vs. flux densities in $R$ band.

2007). The red component is non-thermal optical emission that originates in the relativistic jet. Figure 19 can be interpreted within the assumption that the blue component is constant during our observations both in flux and in spectral index, while the red component varies significantly in flux and slightly (within \pm 0.15 ) in spectral index. The latter can account for the scatter observed within different intervals of averaging. We derive a linear approximation of $\alpha_{\text {cont }}=(0.18 \pm 0.04)+(0.30 \pm 0.02) S_{\mathrm{V}}$ for $S_{\mathrm{V}}$ from 0 to $4.0 \mathrm{mJy}$, which is shown in Figure 19 by a solid line. According to the Pearson's $\chi^{2}$ test the linear fit corresponds to the data sufficiently well with $\chi^{2}=1.83$ for 103 measurements. Vanden Berk et al. (2001) have created a composite continuum spectrum of a quasar using a homogeneous data set consisting of over 2200 quasar spectra from the Sloan Digital Sky Survey (SDSS), which covers a rest-wavelength range from 800 to $8555 \AA$. The quasars were selected from both optical and radio criteria. The authors have found that at wavelengths from $\sim 1300$ to $5000 \AA$ the continuum is well fit by a power law with $\alpha_{v}=0.44$, which we denote as $\alpha_{\text {disk }}$, assuming that this continuum should represent the emission from the accretion disk. Although it is not clear what fraction of quasars in the sample are radio loud, the index is in good agreement with values found in optically selected quasar samples (e.g., Natali et al. 1998). Under the assumption that the accretion disk in 3C 454.3 has similar properties as the disk of a generic quasar and, therefore, when $\alpha_{\text {cont }}=\alpha_{\text {disk }}$ the contribution of the nonthermal component to the continuum is negligible, we can use the linear fit for $\alpha_{\text {cont }}$ to estimate the flux of the accretion disk, $S_{\text {disk }}^{\mathrm{V}}=0.85 \pm 0.15 \mathrm{mJy}$, which corresponds to $S_{\text {disk }}^{\mathrm{R}}=0.91 \pm 0.16 \mathrm{mJy}$. As expected, these values are slightly less than the minimum flux of the quasar observed in $V$ and $R$ bands, respectively.

\subsection{Spectral Properties of Optical and Near-IR Synchrotron Emission}

A method proposed by Hagen-Thorn (1997) allows one to subtract from the total near-IR to UV emission the contribution of components that either are constant or vary on long timescales. These are expected to include the accretion disk, BLR, and dusty torus. The result is a relative SED, RSED, of the component responsible for variable emission on timescales of hours, days, or weeks. Since it is synchrotron emission that varies on such short timescales in a blazar, we refer to this as the "synchrotron component." The method is based on simultaneous multicolor observations and assumes a linear dependence between variations at two different bands, $S_{N}=A_{N}+C_{N} S_{r}$, where $N$ is the band at which the flux is measured, and $S_{r}$ is the flux at a reference band (see below). Figures 20 and 21 reveal approximately linear flux-flux relations at different bands that are statistically significant at a level $\geqslant 99.9 \%$ according to the $\chi^{2}$ criterion. We consider that two measurements at different wavelengths are simultaneous if they are performed within $2 \mathrm{hr}$ of each other. Table 7 shows the number of simultaneous observations at different wavelengths with respect to $R$ band for outbursts I and III, as well as the values of $\chi^{2}$ for a linear fit to the flux-flux relations. Figures 20 and 21 show that the simultaneous measurements cover a wide range of variability during both outbursts. Unfortunately, in the case of outburst II there are no observations in the $U V$ region and only 5-6 observations at near-IR wavelengths over a narrow range of flux levels. $R$ band is chosen as the reference band for the construction of the RSED, since the largest number of observations was obtained in this band. In the case of $U V$ observations, $B$ band serves as a primary reference band (see Figures 20 (left) and 21 (left)) and the linear dependence between the $B$ and $R$ fluxes is used to derive the coefficients $C_{\mathrm{U}}, C_{\mathrm{Uvw} 1}, C_{\mathrm{UvM} 2}$, and $C_{\mathrm{Uvw}}$. Similarly, $J$ band is the primary reference band for measurements in $H$ and $K$ bands. The $C$ coefficients are given in Table 7 . The dependence of the coefficients on frequency represents an RSED. Figure 22 shows the RSED for outbursts I and III. Both RSEDs can be approximated by a power law, $S \propto v^{-\alpha_{\text {opt }}^{\text {syn }}}$, with similar spectral indices, $\alpha_{\mathrm{opt}}^{\mathrm{syn}}=1.77 \pm 0.05$ and $\alpha_{\mathrm{opt}}^{\mathrm{synII}}=1.71 \pm 0.04$. This synchrotron emission represents a red spectral component that dominates the optical continuum when the source is brighter than $S_{\mathrm{V}} \sim 3.5 \mathrm{mJy}$, as discussed in Section 5.2. The spectral index of the red component is significantly steeper than the spectral index of the optical continuum, $\alpha_{\text {cont }} \sim 1.30$, during high optical states, hence the disk emission is always significant for the quasar SED.

\subsection{Spectral Properties of Far-IR Synchrotron Emission}

We have applied the same method as described in Section 5.3 for simultaneous far-IR data obtained during outburst III with 

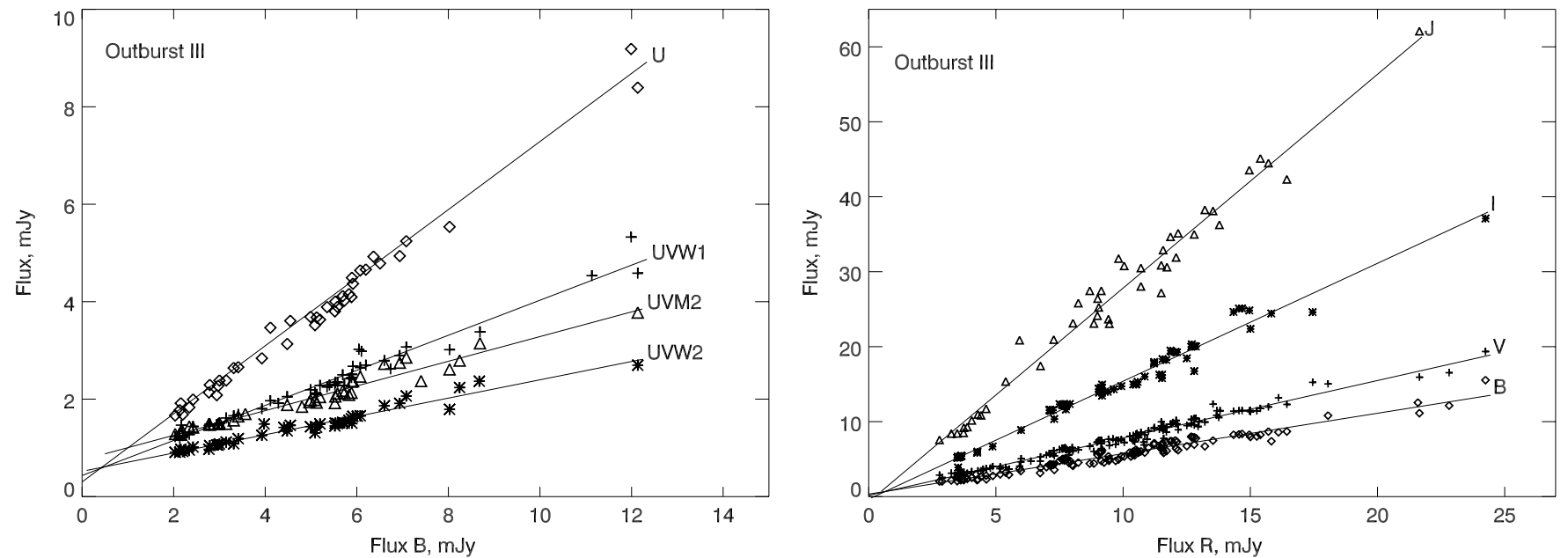

Figure 21. Flux-flux dependences during outburst III for simultaneous measurements at different wavelengths. Left: flux densities at $U V$ bands ( $U$ : diamonds, UVW1: crosses, UVM2: triangles, and UVW2: asterisks) vs. flux densities in $B$ band. Right: flux densities in optical and near-IR bands ( $B$ : diamonds, $V:$ crosses, $I$ : asterisks, and $J$ : triangles) vs. flux densities in $R$ band.

Table 7

Relative Spectral Energy Distribution of Optical/Near-IR Synchrotron Components

\begin{tabular}{|c|c|c|c|c|c|c|c|}
\hline \multirow{2}{*}{$\begin{array}{l}\text { Band } \\
\text { (1) }\end{array}$} & \multirow{2}{*}{$\begin{array}{c}\log _{10}(v) \\
(\mathrm{Hz}) \\
(2)\end{array}$} & \multicolumn{3}{|c|}{ Outburst I } & \multicolumn{3}{|c|}{ Outburst III } \\
\hline & & $\begin{array}{l}N \\
\text { (3) }\end{array}$ & $\begin{array}{c}\log _{10}\left(S_{\text {band }} / S_{\mathrm{R}}\right) \\
(4)\end{array}$ & $\begin{array}{l}\chi^{2} \\
(5)\end{array}$ & $\begin{array}{l}N \\
\text { (6) }\end{array}$ & $\begin{array}{c}\log _{10}\left(S_{\text {band }} / S_{\mathrm{R}}\right) \\
(7)\end{array}$ & $\begin{array}{l}\chi^{2} \\
(8)\end{array}$ \\
\hline UVW2 & 15.169 & 6 & $-0.991 \pm 0.085$ & 0.35 & 37 & $-0.945 \pm 0.031$ & 0.59 \\
\hline UVM2 & 15.128 & 6 & $-0.827 \pm 0.084$ & 1.36 & 44 & $-0.805 \pm 0.029$ & 4.05 \\
\hline UVW1 & 15.056 & 6 & $-0.752 \pm 0.057$ & 0.42 & 36 & $-0.704 \pm 0.022$ & 0.70 \\
\hline$U$ & 14.933 & 6 & $-0.450 \pm 0.062$ & 2.08 & 41 & $-0.422 \pm 0.018$ & 2.52 \\
\hline$B$ & 14.833 & 33 & $-0.313 \pm 0.016$ & 0.73 & 125 & $-0.264 \pm 0.010$ & 0.54 \\
\hline V & 14.736 & 38 & $-0.116 \pm 0.008$ & 0.47 & 147 & $-0.110 \pm 0.009$ & 0.71 \\
\hline$R$ & 14.760 & $\ldots$ & 0.0 & $\ldots$ & $\ldots$ & 0.0 & $\ldots$ \\
\hline$I$ & 14.574 & 21 & $0.173 \pm 0.021$ & 0.57 & 67 & $0.181 \pm 0.010$ & 1.26 \\
\hline$J$ & 14.387 & 25 & $0.467 \pm 0.023$ & 3.93 & 44 & $0.455 \pm 0.012$ & 4.03 \\
\hline$H$ & 14.262 & $\ldots$ & $\ldots$ & $\ldots$ & 9 & $0.705 \pm 0.071$ & 3.30 \\
\hline K & 14.140 & 25 & $0.844 \pm 0.043$ & 9.25 & 44 & $0.811 \pm 0.044$ & 4.21 \\
\hline
\end{tabular}

Notes. Column 1: band of observations; Column 2: logarithm of effective frequency of band; Column 3: number of simultaneous observations during outburst I in given band and $R$ band (in $B$ band for $U V$ filters, in $J$ band for $H$ and $K$ filters); Column 4: $\operatorname{logarithm}$ of the slope of the linear dependence between the flux in a given band and $R$ band and its $1 \sigma$ uncertainty for outburst I; Column 5: the $\chi^{2}$ error statistic for a linear approximation of the flux-flux dependence during outburst I; Columns 6-8: the same as Columns 3-5, respectively, for outburst III.

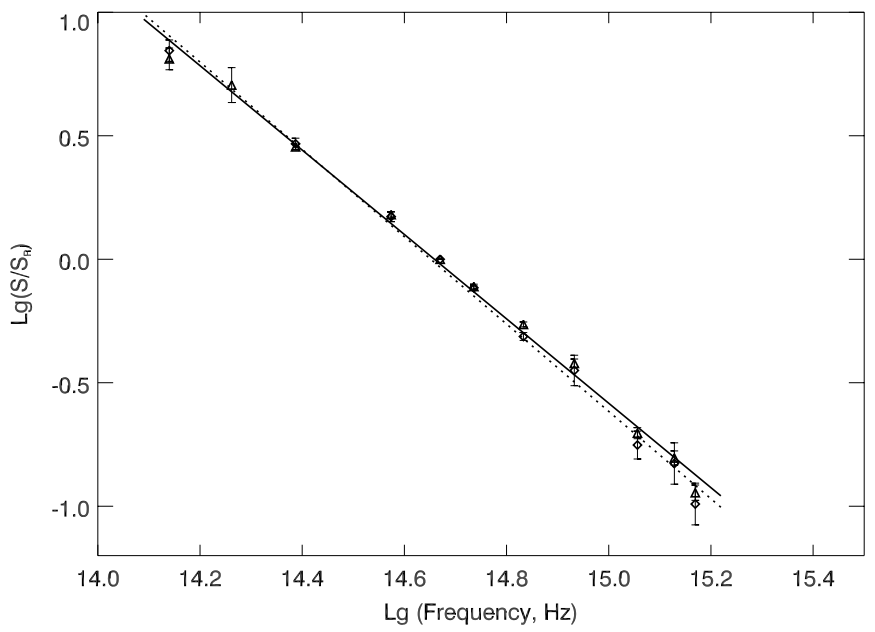

Figure 22. Relative spectral energy distribution of the synchrotron component responsible for the variability at optical and near-IR wavelengths during outbursts I (diamonds and dotted line) and III (triangles and solid line). the Herschel PACS and SPIRE photometers at 70, 160, 250, 350, and $500 \mu \mathrm{m}$, along with 0.85 and $1 \mathrm{~mm}$ measurements obtained with the SMA and IRAM. In this case we treat two observations as simultaneous if they are performed within $24 \mathrm{hr}$ of each other. We use $250 \mu \mathrm{m}$ as a reference wavelength relative to which the RSED is constructed, except for measurements at $70 \mu \mathrm{m}$, for which $160 \mu \mathrm{m}$ serves as a preliminary reference wavelength, with the linear dependence between fluxes at $160 \mu \mathrm{m}$ and $250 \mu \mathrm{m}$ employed to derive $C_{70}$. Figure 23 (left) shows that the flux-flux relations follow linear dependences quite well. Table 8 lists the number of simultaneous observations at different wavelengths, values of $\chi^{2}$ for linear fits, and coefficients $C$ that represent the slopes of the linear fits. The dependence of the coefficients on frequency is plotted in Figure 23 (right), which shows a good correspondence $\left(\chi^{2}=1.19\right)$ of far-IR points (from 70 to $850 \mu \mathrm{m}$ ) with a power law of spectral index $\alpha_{\mathrm{IR}}^{\text {syn }}=$ $0.79 \pm 0.06$. The $1 \mathrm{~mm}$ measurement deviates slightly from the fit, falling a factor of 0.84 below an extrapolation of the fit to the $70-850 \mu \mathrm{m}$ RSED. This deviation most likely indicates that the variable component responsible for the synchrotron emission 

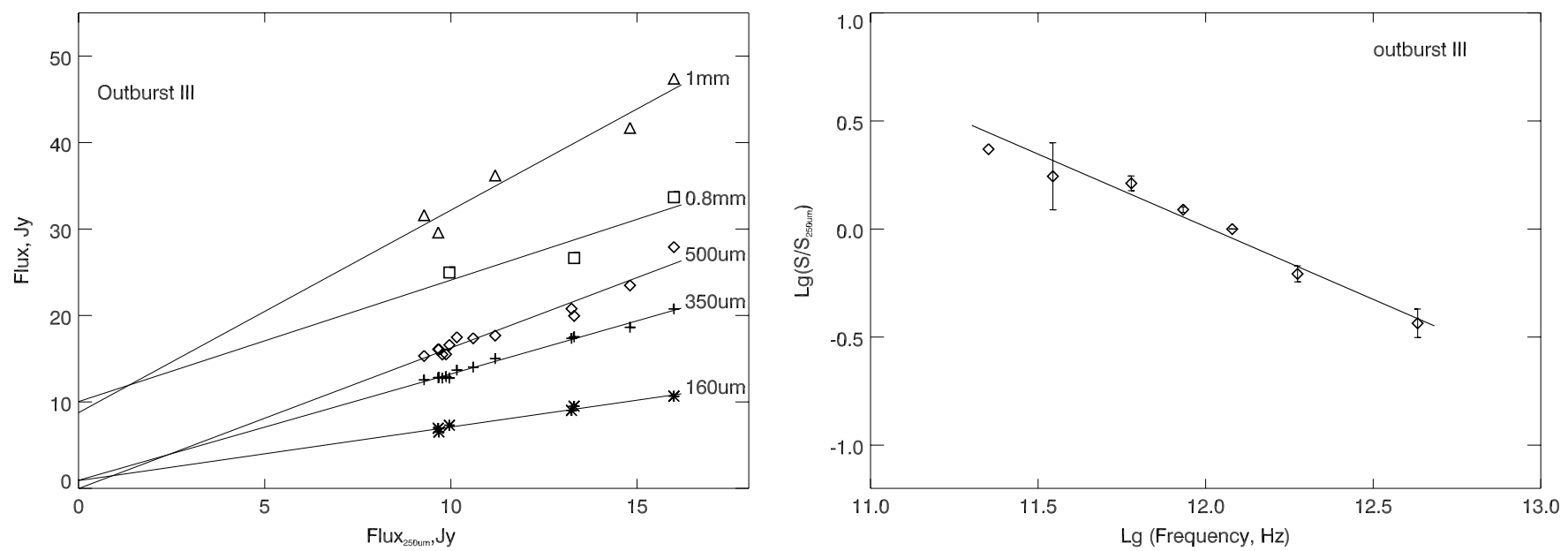

Figure 23. Left: flux-flux dependences for simultaneous measurements at different wavelengths; flux densities at $160 \mu \mathrm{m}$ (asterisks), $350 \mu \mathrm{m}$ (crosses), $500 \mu \mathrm{m}$ (diamonds), $0.85 \mathrm{~mm}$ (squares), and $1.3 \mathrm{~mm}$ (triangles) vs. flux densities at $250 \mu \mathrm{m}$. Right: relative spectral energy distribution of the synchrotron component responsible for the variability at far-IR wavelengths.

Table 8

Relative Spectral Energy Distribution of Far-IR Synchrotron Component during Outburst III

\begin{tabular}{lcccc}
\hline \hline$\lambda$ & $\log _{10}(\nu)$ & $N$ & $\log _{10}\left(S_{\lambda} / S_{250}\right)$ & $(4)$ \\
$(1)$ & $(2)$ & $(3)$ & $(4)$ & $(5)$ \\
\hline $70 \mu \mathrm{m}$ & 12.633 & 15 & $-0.436 \pm 0.066$ & 1.40 \\
$160 \mu \mathrm{m}$ & 12.274 & 6 & $-0.208 \pm 0.037$ & 1.36 \\
$250 \mu \mathrm{m}$ & 12.079 & $\ldots$ & 0.0 & $\ldots$ \\
$350 \mu \mathrm{m}$ & 11.933 & 13 & $0.090 \pm 0.013$ & 0.62 \\
$500 \mu \mathrm{m}$ & 11.778 & 13 & $0.211 \pm 0.033$ & 0.73 \\
$850 \mu \mathrm{m}$ & 11.544 & 3 & $0.244 \pm 0.155$ & 2.22 \\
$1.3 \mathrm{~mm}$ & 11.352 & 5 & $0.370 \pm 0.061$ & 2.07 \\
\hline
\end{tabular}

Notes. Column 1: wavelength of observations; Column 2: logarithm of frequency of observations; Column 3: number of simultaneous observations at a given wavelength and $250 \mu \mathrm{m}$ (at $160 \mu \mathrm{m}$ for $70 \mu \mathrm{m}$ ); Column 4: logarithm of slope of linear dependence between the flux at a given wavelength and $250 \mu \mathrm{m}$ and its $1 \sigma$ uncertainty; Column $5: \chi^{2}$ error statistic for a linear approximation of the flux-flux dependence.

at $1 \mathrm{~mm}$ is partially optically thick. The good correlation between far-IR and $1 \mathrm{~mm}$ flux variations (Wehrle et al. 2012 and Figure 23, left) and between $1 \mathrm{~mm}$ and $7 \mathrm{~mm}$ core flux and polarization behavior (Section 4.2) suggests that the mm-wave $V L B I$ core is the region where the synchrotron far-IR emission originates.

\subsection{Spectral Energy Distributions}

We construct SEDs of the quasar from $U V$ to $\mathrm{mm}$ wavelengths for three epochs during outburst III: on 2010 November 3/4 (RJD: 5503), at the beginning of flare $a$; on November 19 (RJD: 5519), at the maximum of the outburst; and on December 7 (RJD: 5537), during the fading branch of the outburst. We model each SED of the quasar assuming that (1) the optical continuum from $U V$ to near-IR wavelengths consists of a blue component with a constant spectral index $\alpha_{\text {disk }}=0.44$ and constant flux, $S_{\text {disk }}^{\mathrm{V}}=0.85 \mathrm{mJy}$ plus a variable red (synchrotron) component with a constant spectral index $\alpha_{\text {opt }}^{\text {syn }}=1.71$, and (2) the mid- and far-IR continuum is dominated by a variable (synchrotron) component with a constant spectral index $\alpha_{\mathrm{IR}}^{\mathrm{syn}}=0.79$ with a contribution from jet knot $K 09$. We use Herschel observations to determine the minimum wavelength where the contribution from K09 is still signifi-
Table 9

Parameters of SEDs during Outburst III

\begin{tabular}{lccc}
\hline \hline Parameter & Nov $3 / 4$ & Nov 19 & Dec 7 \\
\hline$S_{\mathrm{V}}^{\text {obs }}(\mathrm{mJy})$ & $7.59 \pm 0.28$ & $16.52 \pm 0.15$ & $6.00 \pm 0.12$ \\
$S_{1 \mathrm{~mm}}^{\text {obs }}(\mathrm{Jy})$ & $34.9 \pm 1.8$ & $48.8 \pm 2.40$ & $29.6 \pm 1.5$ \\
$S_{K 09}(\mathrm{Jy})$ & $15.5 \pm 0.3$ & $11.1 \pm 0.2$ & $11.0 \pm 0.2$ \\
$\nu_{\text {peak }}(\mathrm{Hz})$ & $4.66 \mathrm{E}+13$ & $4.78 \mathrm{E}+13$ & $2.39 \mathrm{E}+13$ \\
$\lambda_{\text {peak }}(\mu \mathrm{m})$ & 6.4 & 6.3 & 12.5 \\
$S_{\text {peak }}(\mathrm{mJy})$ & 493 & 1079 & 895 \\
\hline
\end{tabular}

Notes. Parameters of SEDs in the observer's frame- $S_{\mathrm{V}}^{\mathrm{obs}}$ : flux density observed in $V$ band; $S_{1 \mathrm{~mm}}^{\text {obs }}$ : flux density observed at $1.3 \mathrm{~mm}, S_{K 09}$ : flux density of knot $K 09$ at $7 \mathrm{~mm}$; $v_{\text {peak }}$ : frequency of peak of SED, $\lambda_{\text {peak }}$ : wavelength of peak of SED, $S_{\text {peak }}$ : flux density at peak of SED. $L_{\text {disk }}$ : luminosity of accretion disk integrated from 6500 to $2000 \AA$.

cant, $\sim 80 \mu \mathrm{m}$. We determine the flux density of the red variable component in $V$ band as $S_{\mathrm{V}}^{\mathrm{syn}}=S_{\mathrm{V}}^{\mathrm{obs}}-S_{\text {disk }}^{\mathrm{V}}$, where $S_{\mathrm{V}}^{\mathrm{obs}}$ is the observed flux density at a given epoch. The flux density of the variable synchrotron component at $1.3 \mathrm{~mm}$ is calculated as $S_{1 \mathrm{~mm}}^{\mathrm{syn}}=\mathrm{const} \times\left[S_{1 \mathrm{~mm}}^{\mathrm{obs}}-S_{K 09}\left(\nu_{1 \mathrm{~mm}} / \nu_{7 \mathrm{~mm}}\right)^{-\alpha_{K 09}}\right]$, where $S_{K 09}$ is the flux of knot $K 09$ at $7 \mathrm{~mm}$ derived from the VLBA images. We adopt $\alpha_{K 09}=0.7$, and set const $=1.0$ for November $3 / 4$ and 1.19 after November 4 to correct for the higher opacity at mm wavelengths with respect to the far-IR measurements (see Section 5.5). Figure 24 shows the observed and modeled SEDs, as well as SEDs for different components: the variable synchrotron component, the accretion disk, and $K 09$. Table 9 gives the parameters of the SED's peak according to modeling. Using the values of $S_{\text {disk }}^{\mathrm{V}}$ and $\alpha_{\text {disk }}$, we estimate the luminosity of the accretion, $L_{\text {disk }} \approx 2.6 \times 10^{46} \mathrm{erg} \mathrm{s}^{-1}$, with integrating from 6500 to $2000 \AA$.

Figure 24 shows good agreement between the observations and models for all three epochs. Especially promising is the close correspondence between the mid-IR measurements obtained with the IRTF on 2010 November at 4.9, 10.6, and $20.7 \mu \mathrm{m}$ and the modeled SED, since these data-points were not involved in modeling. This supports the assumption that one synchrotron component with a constant spectral index and variable flux is responsible for the outburst at optical/IR wavelengths. The IRTF observations cover wavelengths affected by an IR excess if $3 \mathrm{C} 454.3$ has a dust torus of similar properties 


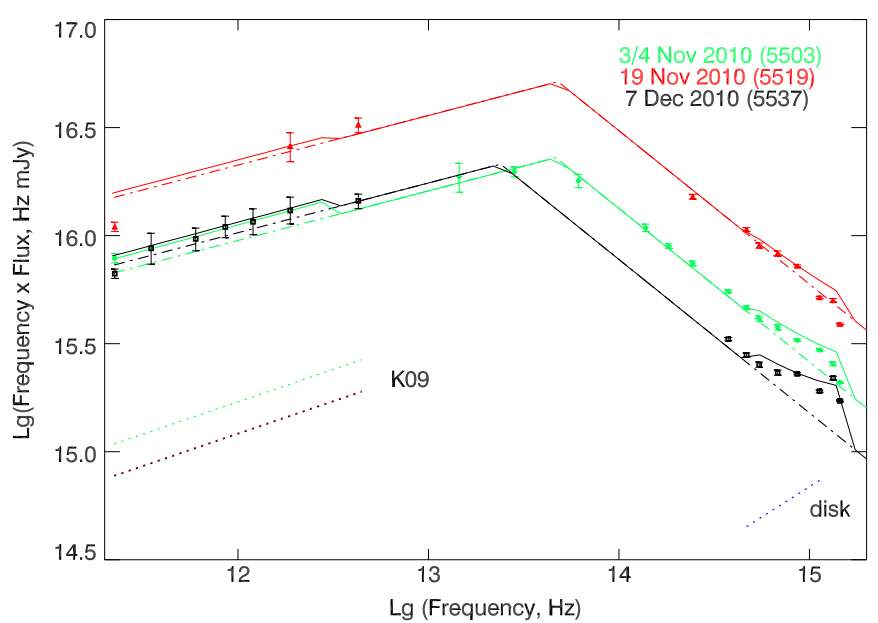

Figure 24. Spectral energy distributions obtained on 2010 November $3 / 4$ (red triangles), November 19 (green diamonds), and December 7 (black squares) and modeled by the sum (solid line) of the emission from a synchrotron component (dash-dotted line), accretion disk (blue dotted line), and knot $K 09$ (black and green dotted lines).

as found in the $\gamma$-ray quasar $1222+216$ (Malmrose et al. 2011). Although neither the IRTF nor Herschel observations suggest the presence of an additional component between 5 and $160 \mu \mathrm{m}$, the measurement at $20.7 \mu \mathrm{m}$-where the peak of a dust component (with temperature $\sim 1200 \mathrm{~K}$ ) is expected in the observer's frame for 3C 454.3 -is slightly higher than the model value. However, this measurement has substantial uncertainties. The maximum deviation from the model flux defines an upper limit to the luminosity of the dust torus of $L_{\text {dust }}<5 \times 10^{46} \mathrm{erg} \mathrm{s}^{-1}$. This is quite high, allowing the dust torus to possess a higher luminosity than that of the accretion disk, while in the case of $1222+216$ IR emission from hot dust has a luminosity of only 0.22 times that of the accretion disk (Malmrose et al. 2011).

The peak of the synchrotron SED corresponds to a break in the spectrum from a spectral index $\alpha_{\text {low }}<1$ to $\alpha_{\text {high }}>1$. The magnitude of the break $\Delta \alpha \equiv \alpha_{\text {high }}-\alpha_{\text {low }}$. During outburst III, $\alpha_{\mathrm{IR}}^{\mathrm{syn}}=0.79$ and $\alpha_{\mathrm{opt}}^{\mathrm{syn}}=1.71$, hence $\Delta \alpha=0.92$. This is considerably greater than the value of 0.5 expected from radiative energy losses while relativistic electrons are constantly injected into the emission region. Interestingly, according to the data listed in Table 9, the wavelength of peak flux, $\lambda_{\text {peak }}$, remains essentially constant as the outburst proceeds to its global maximum on 2010 November 19. If the value of $\lambda_{\text {peak }}$ were determined by a balance between the timescale of radiative losses and the time for the energized electrons to cross the emission region, $\lambda_{\text {peak }}$ would increase as the intensity of seed photons for IC scattering rises. On the other hand, between 2010 November 19 and December $7, \lambda_{\text {peak }}$ increases by a factor of two while the optical flux drops by a factor of 2.75 , consistent with the general trend expected from radiative losses as the rate of injection of relativistic electrons subsides.

Figure 25 presents SEDs of the quasar at the epochs of the $\gamma$-ray maximum, $T_{\gamma}^{\max }$, of each outburst as well as during a more quiescent state. The $\gamma$-ray fluxes during the outbursts are calculated using spectral parameters given in Table 2 of Ackermann et al. (2010) and Table 1 of Abdo et al. (2011) for a LogParabola spectrum. To analyze the SEDs, we apply the Doppler factors derived for knots $K 09$ and $K 10$ (Table 5) for outbursts I and III, respectively. For outburst II we assume $\delta_{\text {II }}$ to be slightly less than $\delta_{\mathrm{I}}$ (Table 10) because, on one hand, the outbursts have very similar SEDs, while on the other hand both

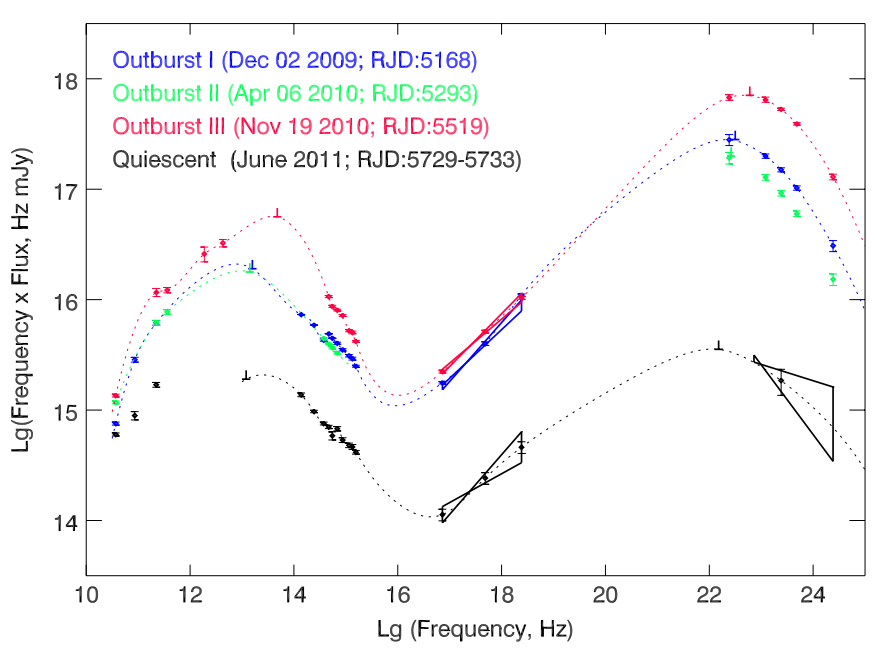

Figure 25. Spectral energy distributions obtained during maxima of outbursts I (blue), II (green), III (red), and at a quiescent state (black); the symbol " $\perp$ ", denotes low- and high-energy peaks of the SEDs according to modeling (see text Section 5.5), while dotted lines show spline approximations of the data points.

Table 10

Parameters of SEDs during Outbursts and Quiescent State

\begin{tabular}{lcccc}
\hline \hline Parameter & Outburst I & Outburst II & Outburst III & Quiescent \\
\hline$\delta$ & 27 & 25 & 51 & 13 \\
$v_{\text {peak }}^{\mathrm{LE}}(\mathrm{Hz})$ & $2.53 \mathrm{E}+13$ & $2.34 \mathrm{E}+13$ & $4.78 \mathrm{E}+13$ & $1.22 \mathrm{E}+13$ \\
$\lambda_{\text {peak }}^{\mathrm{LE}}(\mu \mathrm{m})$ & 11.8 & 12.8 & 6.3 & 24.6 \\
$S_{\text {peak }}^{\mathrm{LE}}(\mathrm{mJy})$ & 760 & 750 & 1100 & 160 \\
$L_{\mathrm{LE}}\left(\mathrm{erg} \mathrm{s}^{-1}\right)$ & $6.9 \mathrm{E}+47$ & $6.8 \mathrm{E}+47$ & $1.9 \mathrm{E}+48$ & $7.0 \mathrm{E}+46$ \\
$L_{\text {syn }}\left(\mathrm{erg} \mathrm{s}^{-1}\right)$ & $8.2 \mathrm{E}+47$ & $6.0 \mathrm{E}+47$ & $1.0 \mathrm{E}+49$ & $4.4 \mathrm{E}+46$ \\
$\nu_{\text {peak }}^{\mathrm{HE}}(\mathrm{Hz})$ & $3.16 \mathrm{E}+22$ & $2.63 \mathrm{E}+22$ & $6.02 \mathrm{E}+22$ & $1.51 \mathrm{E}+22$ \\
$E_{\text {peak }}^{\mathrm{HE}}(\mathrm{GeV})$ & 0.13 & 0.11 & 0.25 & 0.06 \\
$S_{\text {peak }}^{\mathrm{HE}}\left(\mathrm{mJy}^{\mathrm{HE}}\right)$ & $9.0 \mathrm{E}-6$ & $7.6 \mathrm{E}-6$ & $1.2 \mathrm{E}-5$ & $2.3 \mathrm{E}-7$ \\
$L_{\mathrm{HE}}\left(\mathrm{erg} \mathrm{s}^{-1}\right)$ & $1.0 \mathrm{E}+49$ & $7.2 \mathrm{E}+48$ & $2.6 \mathrm{E}+49$ & $1.2 \mathrm{E}+47$ \\
$L_{\mathrm{IC}}\left(\mathrm{erg} \mathrm{s}^{-1}\right)$ & $2.2 \mathrm{E}+48$ & $1.6 \mathrm{E}+48$ & $2.8 \mathrm{E}+49$ & $1.2 \mathrm{E}+47$ \\
\hline
\end{tabular}

the optical and $\gamma$-ray fluxes of outburst II are slightly less than those of outburst I. For the quiescent state we use a minimum Doppler factor obtained by Jorstad et al. (2005) in 1998-2001 when the flux of the VLBI core at $43 \mathrm{GHz}$ did not exceed 5 Jy. All SEDs have a double peak shape typical of blazars with a low-frequency peak $S_{\text {peak }}^{\mathrm{LE}}$ at frequency $v_{\text {peak }}^{\mathrm{LE}}$, representing enhanced synchrotron emission, and a high-frequency peak, $S_{\text {peak }}^{\mathrm{HE}}$ at frequency $v_{\text {peak }}^{\mathrm{HE}}$, which we attribute to inverse Compton scattering. The high-energy peak dominates the SEDs during the active states.

Since neither low- nor high-energy peaks of the SEDs are restricted by the measurements, we use the values of $S_{\text {peak }}$ and $\nu_{\text {peak }}$ for outburst III derived from the modeling (Table 9) and adopt the values of $S_{\text {peak }}^{\mathrm{HE}}$ and $\nu_{\text {peak }}^{\mathrm{HE}}$ for outburst I from the SED presented by Bonnoli et al. (2011) for 2009 December 2. Taking into account that $v=\delta v^{\prime}$, where $v^{\prime}$ is the frequency of the emission emitted by the source and $v$ is the frequency of the emission received by the observer, we have estimated $\nu_{\text {peak }}^{\mathrm{LE}}$ and $v_{\text {peak }}^{\mathrm{HE}}$ for each SED assuming that a change in the Doppler factor is the main factor responsible for the outburst's increase in energy output. Using such peak frequencies, along with spline approximations of the SED's data points, we estimate values of 


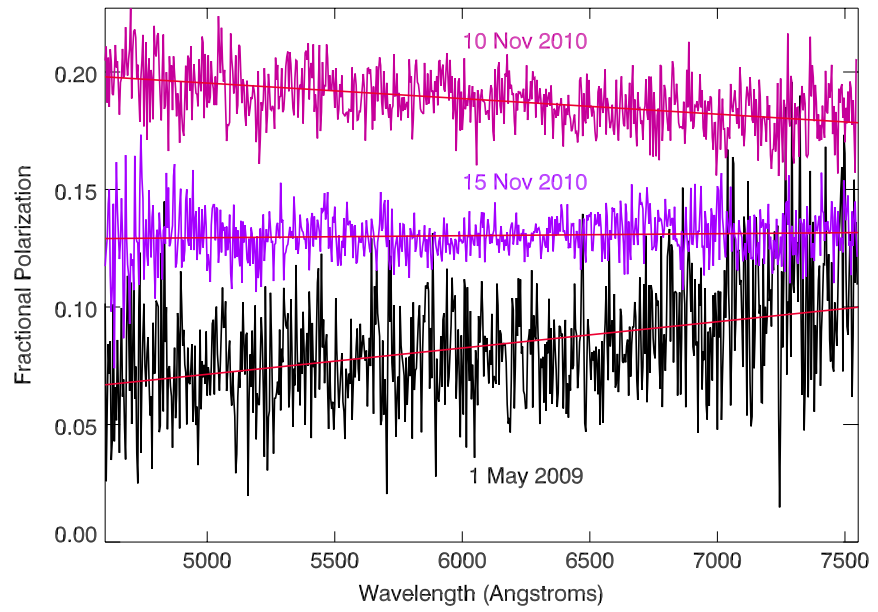

Figure 26. Degree of polarization spectra of 3C 454.3 in the observer's frame at different brightness levels; the red solid lines represent a linear fit of the $P(\lambda)$ dependence.

$S_{\text {peak }}^{\mathrm{LE}}$ and $S_{\text {peak }}^{\mathrm{HE}}$ for each SED. The values are given in Table 10 and marked in Figure 25 by the symbol " $\perp$." Note that in 2011 June the mm-wave emission was still in some moderately active state with respect to the higher energy emission. We calculate the apparent luminosity of $3 \mathrm{C} 454.3$ at the low and high energies for each activity state as $L \approx 4 \pi D_{\mathrm{L}}^{2} S_{\text {peak }} \nu_{\text {peak }}$, where $D_{\mathrm{L}}$ is the luminosity distance, $5.489 \mathrm{Gpc}$. The values of $L^{\mathrm{HE}}$ for the outbursts listed in Table 10 are by a factor of 2-5 lower than those presented by Ackermann et al. (2010) and Abdo et al. (2011) because we use flux densities at the peak frequency only to estimate the luminosity. Table 10 shows that during the quiescent state the luminosity at low energies is comparable to the luminosity of the accretion disk. We estimate the luminosity of a synchrotron component of the emission at the quiescent state as $L_{\mathrm{q}}^{\text {syn }} \approx L_{\mathrm{q}}^{\mathrm{LE}}-L_{\text {disk. Attributing an }}$ enhanced emission at low energies during the outbursts to a change of the Doppler factor, we derive the luminosity of the synchrotron component for each outburst as $L^{\text {syn }} \approx L_{\mathrm{q}}^{\text {syn }}\left(\delta / \delta_{\mathrm{q}}\right)^{4}$. Making the same assumption that enhanced luminosity at high energies is caused by a change of the Doppler factor with respect to the quiescent state and adopting $L_{\mathrm{q}}^{\mathrm{IC}}=L_{\mathrm{q}}^{\mathrm{HE}}$, we calculate values of the luminosity at high energies expected within such assumptions, $L^{\mathrm{IC}} \approx L_{\mathrm{q}}^{\mathrm{IC}}\left(\delta / \delta_{\mathrm{q}}\right)^{4}$. Table 10 shows reasonable agreement between $L^{\mathrm{LE}}$ and $L^{\mathrm{syn}}$, and $L^{\mathrm{HE}}$ and $L^{\mathrm{IC}}$. However, while for outburst III $L^{\mathrm{HE}}$ and $L^{\mathrm{IC}}$ are very consistent, $L^{\text {syn }}$ is larger than $L^{\mathrm{LE}}$ by a factor of 5 . The opposite occurs for outbursts I and II: there is good agreement between $L^{\text {syn }}$ and $L^{\mathrm{LE}}$, while $L^{\mathrm{IC}}$ is underestimated by a factor of five with respect to $L^{\mathrm{HE}}$. Taking into account that the mm-wave emission region is larger than the $\gamma$-ray emission region by a factor of $\sim 100$, the discrepancies suggest slight variations of the Doppler factor within the mm-wave emission region at a given epoch, with a higher $\delta$ than the mean value in the volume where the $\gamma$ ray emission originates. Therefore, it appears that a change in Doppler factor can explain differences in the amplitudes of the outbursts, as was proposed previously by Villata et al. (2007) and Raiteri et al. (2011).

\section{POLARIZATION BEHAVIOR}

Here we analyze our entire set of optical and VLBI polarization data, which were collected from 2008 June to 2011 December. The data set includes 706 measurements of optical polar-

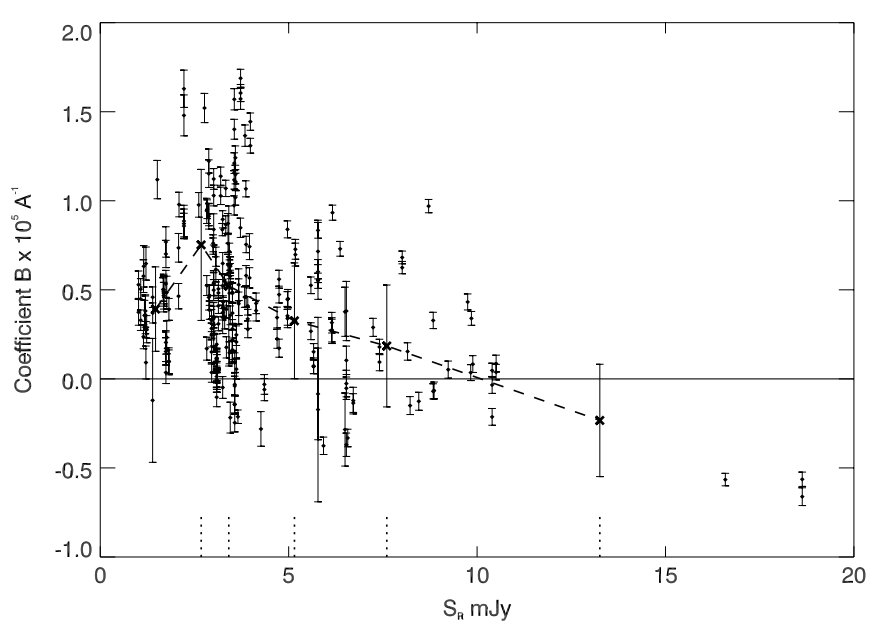

Figure 27. Slope $B$ of $P(\lambda)$ dependence vs. flux density of 3C 454.3 in $R$ band; the dashed line connects the average values of $B$ (crosses); dotted vertical segments show intervals of the averaging; the solid line marks $B=0$.

ization along with simultaneous photometric measurements in $R$ band (390 cases), and 244 spectropolarimetric observations, as well as 52 measurements of polarization in the VLBI core at $43 \mathrm{GHz}$ that coincide with optical observations within 2 days.

\subsection{Dependence of Optical Linear Polarization on Wavelength}

The spectropolarimetric observations performed at Steward Observatory provide spectra of the normalized linear polarization Stokes parameters $q$ and $u$ in the range of 4000-7550 with a dispersion of $4 \AA$. We use the Stokes spectra to calculate the degree of polarization as a function of wavelength, $P(\lambda)=$ $\sqrt{q(\lambda)^{2}+u(\lambda)^{2}}$, within the range of $4500-7000 \AA$, which avoids noisy edges of the $q$ and $u$ spectra. Since not all spectropolarimetric observations were accompanied by photometric measurements in $V$ band, we used the light curve in $R$ band to associate polarization and photometric measurements if they were performed within $3 \mathrm{hr}$ of each other. Figure 26 shows three examples of the $P(\lambda)$ dependence that appear to be representative: (1) the degree of polarization increases with wavelength when the optical emission is weak and $P_{\text {opt }}$ is moderately high (e.g., on 2009 May $1, S_{\mathrm{R}}=1.51 \pm 0.02 \mathrm{mJy}$ and $P_{\mathrm{opt}}=7.94 \% \pm 0.09 \%$ ); (2) the degree of polarization does not depend on wavelength when the optical emission is sufficiently bright and $P_{\mathrm{opt}}$ is high (e.g., on 2010 November $15, S_{\mathrm{R}}=10.51 \pm 0.18 \mathrm{mJy}$ and $P_{\mathrm{opt}}=13.00 \% \pm 0.04 \%$ ); and (3) the degree of polarization decreases with wavelength when the optical emission is very bright and highly polarized (e.g., on 2010 November 10, $S_{\mathrm{R}}=18.63 \pm 0.36 \mathrm{mJy}$ and $\left.P_{\mathrm{opt}}=18.84 \% \pm 0.05 \%\right)$. We use a linear fit, $P(\lambda)=A+B \lambda$, to approximate the dependence of the fractional polarization on wavelength for each spectrum obtained from 2009 April to 2011 August (244 spectra). Examples of the fits are shown in Figure 26 by red solid lines with slope $B$ equal to $(0.112 \pm 0.011) \times 10^{-4} \AA^{-1},(0.38 \pm 0.44) \times$ $10^{-6} \AA^{-1}$, and $(-0.661 \pm 0.050) \times 10^{-5} \AA^{-1}$ for 2009 May, 2010 November 15, and 2010 November 10, respectively. Figure 27 plots derived values of slope $B$ versus brightness in $R$ band for all observations, while Table 6 lists the average values of slope $B$ for different brightness intervals. Figure 27 and Table 6 show that there is a change in the $P(\lambda)$ dependence with brightness: for $S_{\mathrm{R}} \leqslant 4.5 \mathrm{mJy}$ the coefficient $B$ is positivedefinite despite significant scatter, while for $S_{\mathrm{R}}>4.5 \mathrm{mJy} B$ is close to zero. This agrees very well with the finding discussed 

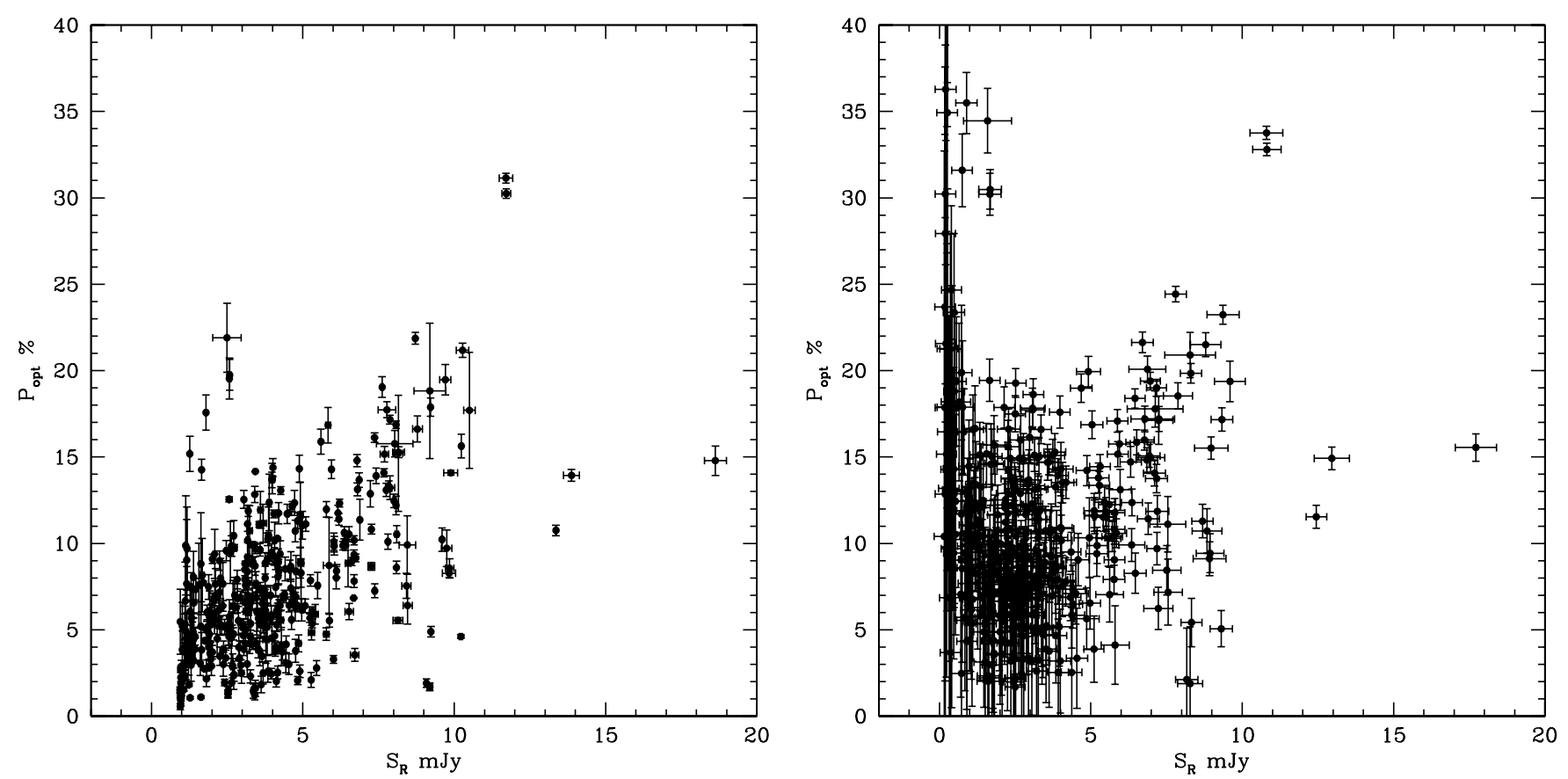

Figure 28. Left: dependence of the degree of observed optical polarization on brightness of the quasar in $R$ band. Right: dependence of the degree of optical polarization of the synchrotron component on its brightness in $R$ band.

in Section 5.2 that the optical continuum consists of two components, blue (BBB) and red (synchrotron). The contribution of the $\mathrm{BBB}$ to the optical emission is significant in the blue part of the spectrum, especially at low flux levels. Dilution by this component leads to a degree of polarization that increases at longer wavelengths (Figure 27), as found previously by Smith et al. (1988). The latter trend suggests that the emission of the BBB is unpolarized. The $P(\lambda)$ dependence disappears when the nonthermal component dominates the total optical emission, as expected for the synchrotron emission over a relatively narrow wavelength range. Figure 27 and Table 6 also show that at very bright flux levels ( $S_{\mathrm{R}}>10 \mathrm{mJy}$ ) $B$ is negative, which implies a higher degree of polarization at shorter wavelengths. Although the number of such observations is small, such a tendency supports models in which electrons are accelerated at a front and then lose energy to radiation. In this case, higher-energy electrons occupy a smaller volume beyond the front-with a more uniform magnetic field-than do lower-energy electrons that radiate at longer wavelengths (e.g., Marscher \& Gear 1985; Marscher et al. 1992; Marscher \& Jorstad 2010). Note that we have investigated dependence of position angle of polarization on wavelength and found that $\chi_{\text {opt }}$ does not depend on $\lambda$, either at high or moderate degrees of polarization, although at a low level of polarization uncertainties of $\chi_{\text {opt }}(\lambda)$ increase significantly.

\subsection{Dependence of Optical Linear Polarization on Brightness}

Figure 28 (left) shows the dependence between the degree of optical polarization and flux of 3C 454.3 in $R$ band. The fractional polarization changes from $<0.5 \%$ to $30 \%$, while the flux varies from $\sim 1 \mathrm{mJy}$ to $20 \mathrm{mJy}$. The Spearman rank correlation coefficient, $\rho=0.565$, gives a statistical significance of $98.7 \%$ that the values are related, with the degree of polarization rising along with the flux. We have shown above that the optical continuum consists of two components: unpolarized thermal (BBB) and polarized synchrotron (jet) emission. According to Section 5.2, the contribution of the BBB in $R$ band is $S_{\mathrm{R}}^{\text {disk }} \sim 0.91 \mathrm{mJy}$. If we assume that the thermal component was constant during our observations and completely unpolarized, we can derive the flux and degree of polarization of the variable synchrotron component: $S_{\mathrm{R}}^{\mathrm{var}}=S_{\mathrm{R}}^{\text {obs }}-S_{\mathrm{R}}^{\text {disk }}$ and $P_{\mathrm{opt}}^{\mathrm{var}}=P_{\mathrm{opt}} \times S_{\mathrm{R}}^{\mathrm{obs}} / S_{\mathrm{R}}^{\mathrm{var}}$ with $S_{\mathrm{R}}^{\text {disk }}=0.91 \pm 0.16 \mathrm{mJy}$ (Section 5.2). Figure 28 (right) shows the dependence between the degree of optical polarization and flux for the variable component. In this case an increase of the degree of polarization is observed also at a very small flux level of the variable source, with $P_{\mathrm{opt}}^{\mathrm{var}}$ rising up to $\sim 40 \%$ while $S_{\mathrm{var}}<1 \mathrm{mJy}$, although the uncertainties in $P_{\mathrm{opt}}^{\mathrm{var}}$ are significant owning to an uncertainty in the derived BBB flux. An increase of the degree of polarization along with the flux level might be a signature of shock processes owing to ordering of the magnetic field in the shocked region if the quiescent jet has a chaotic magnetic field. However, Figure 28 (right) suggests that in a completely quiescent state the synchrotron component originates in a region with a very wellordered magnetic field. This implies that the optical synchrotron emission during quiescent and active states arises from different regions in the jet. These two synchrotron components (quiescent and active) perhaps possess different polarization properties that can explain the minimum values of $P_{\mathrm{opt}}^{\mathrm{var}}$ at fluxes of 2-3 mJy when a quiescent synchrotron component has a flux comparable to that of an active synchrotron component at a moderate stage of activity. A "competition" between the thermal and different synchrotron components at a moderate flux level might be responsible also for the largest scatter in the slope of the $P(\lambda)$ dependence seen at flux level between 2 and 4 mJy (Table 6).

If the optical synchrotron emission originates in different regions during quiescent and active states, we can expect that the magnetic field configuration of the regions is different. Figures 29 show the distributions of the position angle of optical polarization with respect to the jet direction for $S_{\mathrm{var}}<1 \mathrm{mJy}$ (left) and $S_{\mathrm{var}}>5 \mathrm{mJy}$ (right). It is clear that the position angle of polarization differs during quiescent and active states, with $\chi_{\text {opt }}$ tending to align nearly along the jet direction during 

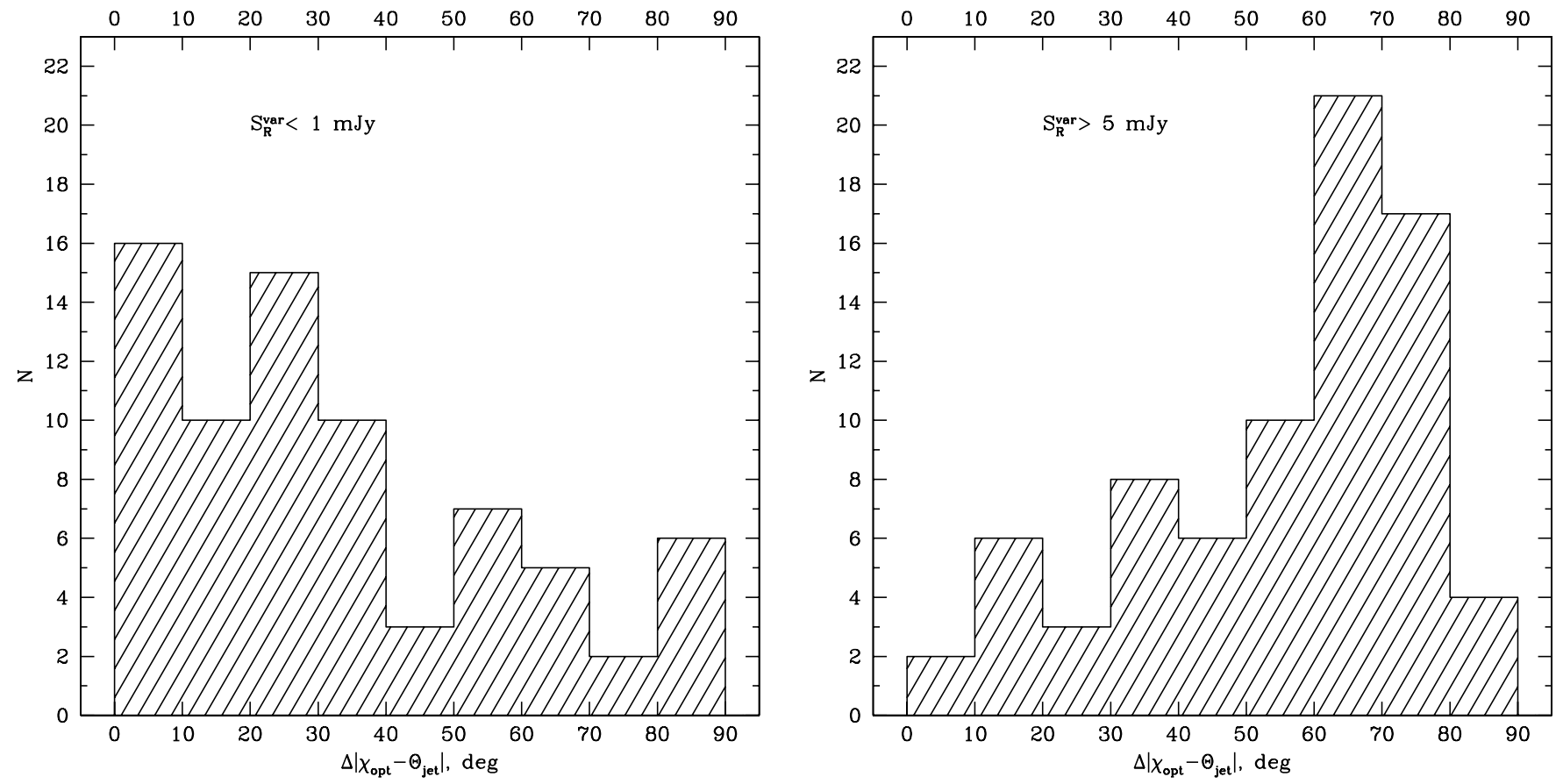

Figure 29. Distribution of position angle of optical polarization with respect to jet axis during low (left) and high (right) levels of optical synchrotron emission.

quiescent states, while being oblique, or nearly perpendicular to the jet direction, during active states. This suggests that the optical synchrotron emission during a quiescent state originates in a region with a well-ordered toroidal magnetic field. Such a region is most likely located in the magnetically dominated part of the jet, relatively close to the black hole (within several thousand Schwarzschild radii; Meier et al. 2000; McKinney 2006). On the other hand, the optical synchrotron emission during an active state originates in a region with a chaotic magnetic field, although a further increase of the flux leads to ordering the magnetic field along the jet axis. The latter implies that the region of flaring synchrotron emission is located farther downstream the jet where either spiral loops of the toroidal magnetic field are very loose (Lyutikov et al. 2005) or the effects of velocity shear align the magnetic field with the jet axis (Laing 1980; D'Arcangelo et al. 2009).

\subsection{Comparative Analysis of Optical and Millimeter-wave Polarization during Gamma-Ray Outbursts}

We compare the position angle of the polarization (EVPA) at optical wavelengths and in the VLBI core at $43 \mathrm{GHz}$ for simultaneous observations. Figure 30 shows the distribution of differences between the optical EVPA, $\chi_{\mathrm{opt}}$, and EVPA in the VLBI core, $\chi_{\text {core }}$. The distribution is bimodal, with $\chi_{\text {opt }}$ either similar to $\chi_{\text {core }}$ or different by $>50^{\circ}$. Note that a good agreement between the position angles, $\left|\chi_{\text {opt }}-\chi_{\text {core }}\right|<20^{\circ}$ (20 cases), is observed when the source is brighter than $2 \mathrm{mJy}$ in $R$ band.

The distribution therefore suggests that there may be a relationship between the properties of optical and VLBI core polarization when the source is in an active state. We find stronger evidence for such a connection in observed similarities between optical polarization parameters and those in the core during outbursts. Figure 31 shows the parameters of the optical and core polarization during outbursts I, II, and III (we also use the polarization data obtained in $V$ band by Sasada et al. 2012 during outburst I). The data for each outburst are plotted relative to the corresponding time of $\gamma$-ray flux maximum,

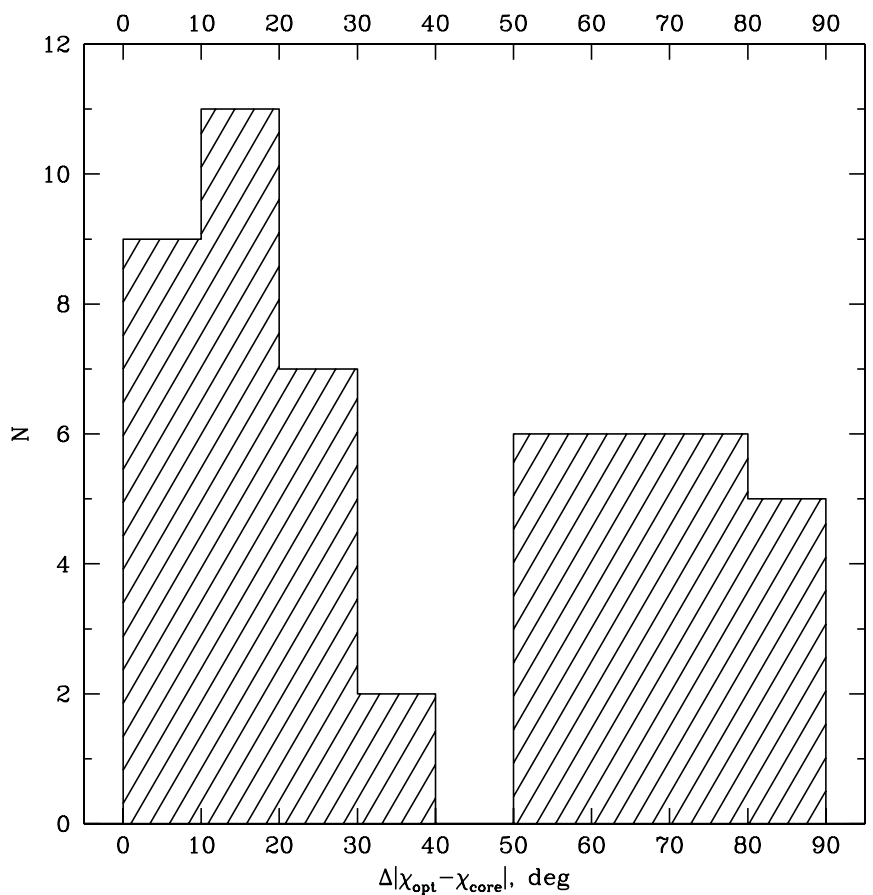

Figure 30. Distribution of offsets between position angle of optical polarization and position angle of polarization in the VLBI core at $7 \mathrm{~mm}$.

$T_{\gamma}^{\max }$, listed in Table 1. In general, there is an increase of $P_{\mathrm{opt}}$ during the outbursts. However, measurements at the peak of outbursts I and II reveal a significant drop of the degree of optical polarization (down to $2 \%-3 \%$ ) over a period of $\sim 3-4$ days centered at $T_{\gamma}^{\max }$. The degree of polarization in the core reveals similar behavior: $P_{\text {core }}$ increases during outbursts up to $4 \%$, but it drops below $1 \%$ close to $T_{\gamma}^{\max }$ for outburst II, for which there are observations within 2 days of $T_{\gamma}^{\max }$.

The behavior of the position angle of polarization during outbursts is extremely interesting. Figure 31 shows that: (1) 


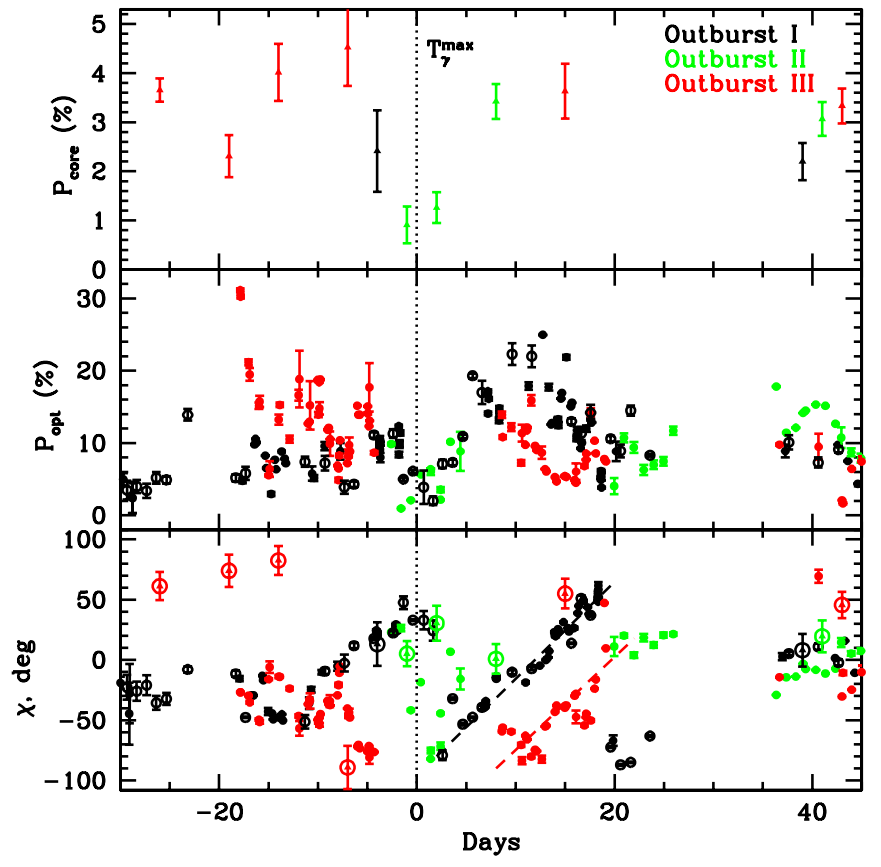

Figure 31. Millimeter-wave core and optical polarization parameters during outburst I (black, open circles are measurements from Sasada et al. 2012), II (green), and III (red) vs. time relative to $T_{\gamma}^{\max }$ of each outburst; from the top: degree of polarization in the core, degree of optical polarization, and position angle of optical polarization (circles) and in the core (triangles inside of circles); the dashed lines show a rotation of $\chi_{\text {opt }}$ during outbursts I (black) and III (red).

near the beginning of an outburst, $\chi_{\text {opt }}$ is relatively stable at $\sim-25^{\circ}$, while $\chi_{\text {core }}$ differs from $\chi_{\text {opt }}$ by $\sim 90^{\circ}$ for outburst III, which indicates that the core is most likely optically thick at $43 \mathrm{GHz}$; (2) $\sim 10$ days before $T_{\gamma}^{\max }$, $\chi_{\text {opt }}$ starts to rotate, although it does so in opposite directions for outbursts I and III; (3) at the peak of a $\gamma$-ray outburst $\chi_{\text {opt }}$ fluctuates on a timescale of several hours; (4) 5-10 days after $T_{\gamma}^{\max }, \chi_{\text {opt }}$ starts a new cycle of rotation with the same counter-clockwise direction for both outbursts I and III, and at a similar rate of $\sim 9^{\circ}$ day $^{-1}$ over at least $10-15$ days; (5) despite similarities in the rotation of $\chi_{\mathrm{opt}}$, there is a constant offset of $\sim 40^{\circ}$ between the rotation lines (the dashed lines in Figure 31, bottom panel); this corresponds to a shift in the directions of the trajectories of knots $K 09$ and $K 10$ within 0.2 mas of the core (see Figure 12 ); (6) $\sim 35$ days after $T_{\gamma}^{\max }$, the optical EVPA stabilizes at $\chi_{\text {opt }} \sim 0^{\circ}$, which is the EVPA of the core as well as that of $K 09$ and $K 10$ when they first appear in the jet (see Figure 14). Therefore, although the optical polarization varies dramatically during $\gamma$-ray outbursts, the behavior of the optical polarization maintains a tight connection with the properties of the mm-wave core and superluminal knots $K 09$ and $K 10$.

\section{DISCUSSION}

The multi-frequency outbursts of the quasar 3C 454.3 in 2009 and 2010 have been analyzed by different authors. Bonnoli et al. (2011) have modeled simultaneous SEDs at different stages of outburst I. These authors use a leptonic, one-zone synchrotron and inverse Compton model discussed in detail by Ghisellini \& Tavecchio (2009). They successfully reproduce the large variations in the $\gamma$-ray flux by varying the power injected in relativistic electrons (by a factor of 10 from the quiescent to the highest state), and the bulk Lorentz factor (from 15 to 20). They place the dissipation zone of the outburst within the
BLR ( $\sim 1000$ Schwarzschild radii). Bonnoli et al. (2011) find it necessary to decrease the value of the magnetic field as the outburst progresses to fit the X-ray spectrum. This increases with respect to the $\mathrm{BH}$ the location of the dissipation region during the highest state although by less than a factor of 1.4. A one-zone leptonic model has been used also by Vercellone et al. (2010), Pacciani et al. (2010), and Vercellone et al. (2011) to explain the dynamic behavior of the SEDs during outbursts I and III. The authors employ the synchrotron, SSC, and EC mechanisms, with seed photons for IC scattering provided by the accretion disk and BLR. The scattering is produced by a blob of relativistic plasma moving with $\Gamma \sim 20-25$ at $\sim 0.05 \mathrm{pc}$ from the BH. These authors reproduce the SEDs of the quasar rather well. However, our findings, summarized below, challenge onezone leptonic models that place the dissipation zone of outbursts so close to the BH. Either the BLR of the quasar has a different geometry than assumed, as proposed by León-Tavares et al. (2013), or more complicated models are needed to explain the multi-frequency behavior of 3C 454.3 during outbursts.

We find that the correlation analysis of the high-energy, optical, IR, and mm-wave variations observed in 3C 454.3 in 2009-2011 indicates that the events seen at different wavelengths were co-spatial. However, the size of the emission regions is different at different wavelengths, with the $\gamma$-ray radiation occupying the smallest volume. The behavior of the optical polarization along with the $43 \mathrm{GHz}$ polarization in the parsec scale jet imply that (1) the degree of optical polarization tends to increase when the flux in $R$ band is less than $\sim 2 \mathrm{mJy}$ (a quiescent state) and more than $\sim 4 \mathrm{mJy}$ (an active state); (2) during a quiescent state the position angle of optical polarization tends to align with the jet direction, which suggests a toroidal configuration of the magnetic field; (3) there is better agreement between the optical and VLBI core polarization parameters during active states with both optical polarization angle and EVPA in the core having a preferred direction-perpendicular to the jet axis; and (4) the optical synchrotron emission during quiescent states originates in a location where the magnetic field is well ordered, perhaps in the acceleration and collimation zone (ACZ) upstream of the mm-wave core, while during active states the location of the optical synchrotron emission moves down the jet, closer to the VLBI core.

We find that the VLBI core of the parsec scale jet was active during all three high-energy events, and that the two events corresponding to the most dramatic $\gamma$-ray outbursts were accompanied by the ejection of superluminal knots with the highest Lorentz factor of $\sim 30$ corresponding to the most dramatic $\gamma$-ray outburst. These emission properties and connections cause us to place the event's site within the mm-wave VLBI core located $\sim 20 \mathrm{pc}$ from the BH. The triple-peaked profile of the light curves during each outburst implies that the core with the angular size of $0.05 \pm 0.02$ mas contains three locations where the emission reaches a local maximum. In fact, Jorstad et al. (2010) found evidence for such triple structure in "super-resolved" $43 \mathrm{GHz}$ VLBA images of 3C 454.3. We discuss below three possible theoretical models that could be compatible with this general picture, although each requires future detailed computations to verify how well they can reproduce the observed behavior of 3C 454.3 .

1. Recollimation shocks and the turbulent extreme multi-zone (TEMZ) model. In this model, we associate the triple structure of the core with a system of three alternating conical "recollimation" shocks and rarefactions, as suggested by Daly \& Marscher (1988), Gómez et al. (1997), 
Komissarov \& Falle (1997), Marscher (2006), and Cawthorne (2006). The outbursts occur as a disturbance-an increase in the energy and/or velocity of the flow in the jet, presumably originating at the input site at the jet base-crosses these standing shocks. The disturbance may correspond to a moving shock, but this is not a general requirement. Each standing shock increases the density, compresses the magnetic field component parallel to the shock front, and accelerates particles. The level of and variations in linear polarization suggest that the magnetic field direction varies across the emitting region, as expected if the jet plasma is turbulent. The TEMZ model (Marscher 2012, 2013) calculates the emission expected from such a turbulent plasma flowing down the jet after it crosses a single standing conical recollimation shock in the $\mathrm{mm}$-wave core. The shock energizes electrons and compresses the plasma, leading to strong emission downstream of the shock. The TEMZ code computes the SED from synchrotron radiation and inverse Compton scattering, as well as the linear polarization of the synchrotron emission at various frequencies, as a function of both time and location within the jet. The model for 3C 454.3 assumes a randomly oriented magnetic field upstream of the shock, with each cell (after compression by the shock) having its own field direction and maximum electron energy. The energy density at the jet input varies with time stochastically within a power spectrum of a power-law shape with a slope of -1.2 , similar to that observed for $\gamma$-ray flux variations (Larsson 2013). The plasma in each cell has a velocity that is the vector sum of the general flow and local turbulent velocities. Seed photons for the scattering include infrared emission by hot dust in a parsec-scale molecular torus, as well as synchrotron and inverse Compton photons from a Mach disk on the jet axis. The inclusion of a turbulent magnetic field and non-uniform maximum electron energy across the emission region reproduces in a general manner the fluctuations in polarization and flux observed in 3C 454.3 at different wavelengths (Marscher 2012, 2013). Wehrle et al. (2012) use the TEMZ code to fit several SEDs of 3C 454.3 during outburst III. The derived SEDs match the millimeter to optical and $\gamma$-ray spectra quite well, although the observed X-ray spectrum is somewhat steeper than in the model calculations that challenge the model. In addition, the dust would need to have a luminosity $\sim 1 \times 10^{46} \mathrm{erg} \mathrm{s}^{-1}$, which is half the luminosity of the accretion disk. The distribution of dust would also need to be very patchy in order to extend over a large enough volume to provide a high density of seed photons at distances $\sim 15-20$ pc from the BH. However, in the TEMZ model, the emission at different frequencies occupies a volume whose size is inversely related to the frequency of observation. This dictates that the average degree of linear polarization, as well as the level of variability of both the flux and polarization, should increase with frequency (Marscher 2013). Indeed, we see such a behavior during the outbursts (see Tables 1-4 and Figure 26).

2. Mini-jet model. In the "mini-jet" scenario (Begelman et al. 2008; Giannios et al. 2009; Giannios 2013) compact emitting regions (blobs) move relativistically with a Lorentz factor $\sim 100$ within a jet with a bulk Lorentz factor $\sim 10$. Such extremely fast motions are possible in a magnetically dominated flow where magnetohydrodynamical waves approach the speed of light and a substantial fraction of the jet luminosity is dissipated in reconnection events. In addition, the beaming can be supplemented by an anisotropic electron distribution, such that the electrons stream toward the line of sight in some of the blobs (Cerutti et al. 2012). Although the model has been proposed to explain dramatic $\mathrm{TeV}$ energy variability on a timescale of $<1 \mathrm{hr}$ in some BL Lac objects, it can be adapted to 3C 454.3 with less severe constraints on the Lorentz factors. The model does not depend significantly on the location of the dissipation zone. The main constraint is connected with the timescale for the reconnection to occur, which must be shorter than the observed timescale of the variability, $<3 \mathrm{hr}$ in the case of 3C 454.3. The triple structure of the outburst light curves and core would require three different physical locations where magnetic reconnections occur.

3. Current-driven instability (CDI). According to analytical studies (e.g., Vlahakis \& Königl 2004) and numerical simulations (e.g., McKinney 2006; Tchekhovskoy et al. 2008), relativistic jets are accelerated by magnetic stresses in an extended region dominated by the Poynting flux that do not operate uniformly across the jet radius. This creates a gradient in the bulk Lorentz factor with distance from the jet axis. Magnetically dominated plasma with a toroidal magnetic field is known to be subject to CDI. Narayan \& Tchekhovskoy (2009) find that the sign of the poloidal velocity shear is important for stability of the jet: jets with positive velocity shear (Lorentz factor increasing with radius) are stable, while jets with velocity shear changing sign are unstable. Nalewajko \& Begelman (2012) identify two types of unstable modes, exponential and overstable, and show that the growth rates of exponential modes decrease with increasing velocity shear. These authors note that their results are most suitable at distance scales beyond the main $\mathrm{ACZ}$, where the effects of velocity shear are expected to be most prominent. In 3C 454.3 the position angle of optical polarization and polarization in the VLBI core during active states is predominantly perpendicular to the jet, so that the mean magnetic field is parallel to the jet. Such a configuration of the magnetic field is expected if velocity shear stretches and orders the field lines along the flow (Laing 1980; D'Arcangelo et al. 2009). Nalewajko \& Begelman (2012) propose that CDI can provide an important energy dissipation mechanism and play a crucial role in converting a magnetically dominated jet into a matter-dominated flow that produces the observed emission from blazars. The polarization behavior of 3C 454.3 suggests that the mm-wave core is located at the end of the ACZ. A similar conclusion was drawn from polarization studies of a number of blazars at optical and $\mathrm{mm}$ wavelengths by Jorstad et al. (2007). They suggested that the ACZ ends between the VLBI cores at 3 and $7 \mathrm{~mm}$, which might also be the radiative dissipation zone.

These three models are not necessarily mutually exclusive, i.e., more than one physical mechanism might be operating within the core. For example, CDI could cause the plasma to become turbulent downstream of the jet ACZ, after which the plasma crosses recollimation shocks; or the turbulence instigated by CDI could create the conditions under which magnetic reconnection events are common. The models are all potentially capable of explaining how the timescale of variability at optical and $\gamma$-ray frequencies can be as short as hours for emission arising in the parsec-scale jet. One factor is that the jet is very narrow, with an opening half-angle of $0.014 \mathrm{rad}$ 
(Jorstad et al. 2005). The width of the parsec-scale jet is therefore of order $10^{17} \mathrm{~cm}$. Taking into account polarization properties of the quasar, the size of a turbulent cell or reconnection region could be $\sim 0.1$ times this width. Turbulent motion could enhance the Doppler factor $\delta_{\mathrm{c}}$ of a cell of plasma (Narayan $\&$ Piran 2012) above the mean value $(\sim 30)$, as could fast streams originating in magnetic reconnections (Begelman et al. 2008; Giannios et al. 2009; Giannios 2013). The timescale of flux variability resulting from these factors can be as short as $\tau_{\text {var }}<10^{16}(1+z) /\left(c \delta_{\mathrm{c}}\right) \lesssim 5.7 \mathrm{hr}$, compatible with the minimum observed value.

\section{CONCLUSIONS}

We have discovered a repeating pattern in the properties of the three major $\gamma$-ray plus lower frequency outbursts observed in 3C 454.3 from 2009 to 2011. The duration, shape, and timescale of variability are similar, although the amplitudes of $\gamma$-ray outbursts are different (Table 1). This strongly suggests that the mechanism(s) and location of the high-energy events are the same for all three outbursts. The $\gamma$-ray variations are strongly correlated with those at optical, far-IR, and mmwavelengths with a delay $<1$ day (see also Wehrle et al. 2012), although the timescale of $\gamma$-ray variability is significantly shorter than at longer wavelengths. We have determined that a single synchrotron component is responsible for the variability from UV to IR wavelengths during an outburst (Figure 24), and that the properties of this component-spectral index (Figure 22), timescale of variability (Table 3 ), and polarization parameters (Figure 31) - are similar for the different outbursts. We have found interesting optical polarization behavior during the outbursts that has not been noted previously: despite a general increase in the degree of polarization during an outburst, the degree of polarization drops significantly at the peak of the $\gamma$-ray event. In addition, the position angle of polarization varies on a timescale comparable to that of the $\gamma$-ray flux variations. This argues in favor of turbulence playing a significant role in the variations near the peak of a $\gamma$-ray event.

We have detected apparent superluminal disturbances (knots) in the parcec-scale jet that we associate with the outbursts based on an analysis of the motions of the knots. We have found that the duration of the outbursts matches the time needed for a knot to pass through the mm-wave VLBI core. We have derived the Doppler factors of the knots and shown that the differences in the Doppler factors can explain differences in the amplitudes of the outbursts. We have also shown that the polarization properties of the core and knots, as well as the trajectories of the knots, are connected with the optical polarization properties during the outbursts (Figure 31).

Our multi-frequency analysis shows that, in the absence of relativistic boosting, the luminosity of the quasar 3C 454.3 would be dominated by accretion disk emission, in accordance with the unified scheme of AGNs. The dramatic outbursts from radio wavelengths to $\gamma$-rays are certainly connected with the relativistic jet. The multi-frequency variability along with analysis of the parsec scale jet behavior favor the localization of the outbursts in the mm-wave VLBI core, which is most likely located at the end of the acceleration zone where the magnetically dominated jet is converted into a matter-dominated jet (Marscher et al. 2008; Jorstad et al. 2007).

The research at Boston University (BU) was funded in part by NASA Fermi Guest Investigator grants NNX08AV65G, NNX10AO59G, NNX10AU15G, NNX11AO37G, and
NNX11AQ03G and Swift Guest Investigator grant NNX10AF88G. The VLBA is operated by the National Radio Astronomy Observatory. The National Radio Astronomy Observatory is a facility of the National Science Foundation operated under cooperative agreement by Associated Universities, Inc. The PRISM camera at Lowell Observatory was developed by K. Janes et al. at BU and Lowell Observatory, with funding from the NSF, BU, and Lowell Observatory. The Liverpool Telescope is operated on the island of La Palma by Liverpool John Moores University in the Spanish Observatorio del Roque de los Muchachos of the Instituto de Astrofisica de Canarias, with funding from the UK Science and Technology Facilities Council. The effort at Steward Observatory was funded in part by NASA through Fermi Guest Investigator grant NNX08AW56G and NNX09AU93G. The St. Petersburg State University team acknowledges support from RFBR grants 12-02-00452 and 12-02-31193. The research at the IAA-CSIC is supported by the Spanish Ministry of Economy and Competitiveness and the Regional Government of Andalucía (Spain) through grants AYA2010-14844 and P09-FQM-4784, respectively. A. Wehrle acknowledges Guest Investigator support from NASA via Herschel RSA 1427799 and Fermi Guest Investigator grant NNX11AAO85G. The Submillimeter Array is a joint project between the Smithsonian Astrophysical Observatory and the Academia Sinica Institute of Astronomy and Astrophysics, and is funded by the Smithsonian Institution and the Academia Sinica. The Abastumani team thanks the Georgian National Science Foundation for support through grant GNSF/ST09/4-521. The Swift effort at PSU is supported by NASA contract NAS500136. The Calar Alto Observatory is jointly operated by the Max-Planck-Institut für Astronomie and the Instituto de Astrofísica de Andalucía-CSIC. The IRAM $30 \mathrm{~m}$ telescope is supported by INSU/CNRS (France), MPG (Germany), and IGN (Spain). The Metsähovi team acknowledges support from the Academy of Finland. This study is partly based on data taken and assembled by the WEBT collaboration and stored in the WEBT archive at the Osservatorio Astronomico di Torino-INAF (http://www.oato.inaf.it/blazars/webt/). This paper has made use of up-to-date SMARTS optical/near-infrared light curves that are available at www.astro.yale.edu/smarts/glast/home.php.

Facilities: Fermi, VLBA, EVLA, SMA, Swift, Herschel, IRTF, IRAM:30m, Perkins, Liverpool:2m, Bok, SO:Kuiper, CAO:2.2m, Metsähovi Radio Observatory, WEBT

\section{REFERENCES}

Abdo, A. A., Ackermann, M., Ajello, M., et al. 2011, ApJL, 733, L26 Ackermann, M., Ajello, M., Baldini, L., et al. 2010, ApJ, 721, 1383 Agudo, I., Jorstad, S. G., Marscher, A. P., et al. 2011a, ApJL, 726, L13 Agudo, I., Marscher, A. P., Jorstad, S. G., et al. 2011b, ApJL, 735, L10 Agudo, I., Thum, C., Wiesemeyer, H., \& Krichbaum, T. P. 2010, ApJS, 189, 1 Algaba, J. C., Gabuzda, D. C., \& Smith, P. S. 2011, MNRAS, 411, 85 Begelman, M. C., Fabian, A. C., \& Rees, M. J. 2008, MNRAS, 384, L19 Blandford, R. D., \& Königl, A. 1979, ApJ, 232, 34

Bonning, E., Urry, C. M., Bailyn, C., et al. 2012, ApJ, 756, 13 Bonnoli, G., Ghisellini, G., Foschini, L., Tavecchio, F., \& Ghirlanda, G. 2011, MNRAS, 410, 368

Burbidge, G. R., Jones, T. W., \& Odell, S. L. 1974, ApJ, 193, 43

Cardelli, J. A., Clayton, G. C., \& Mathis, J. S. 1989, ApJ, 345, 245

Cawthorne, T. V. 2006, MNRAS, 367, 851

Cerutti, B., Werner, G. R., Uzdensky, D. A., \& Begelman, M. C. 2012, ApJL, 754, L33

Chatterjee, R., Bailyn, C. D., Bonning, E. W., et al. 2012, ApJ, 749, 191

Chatterjee, R., Jorstad, S. G., Marscher, A. P., et al. 2008, ApJ, 689, 79

Daly, R. A., \& Marscher, A. P. 1988, ApJ, 334, 539

D’Arcangelo, F. D., Marscher, A. P., Jorstad, S. G., et al. 2009, ApJ, 697, 985

Edelson, R. A., \& Krolik, J. H. 1988, ApJ, 333, 646 
Foschini, L., Ghisellini, G., Tavecchio, F., Bonnoli, G., \& Stamerra, A. 2011, A\&A, 530, A77

Ghisellini, G., \& Tavecchio, F. 2009, MNRAS, 397, 985

Giannios, D. 2013, MNRAS, 431, 355

Giannios, D., Uzdensky, D. A., \& Begelman, M. C. 2009, MNRAS, 395, L29

Gómez, J. L., Marscher, A. P., Alberdi, A., Jorstad, S. G., \& Agudo, I. 2002, VLBA Scientific Memo 30 (NRAO)

Gómez, J. L., Martí, J. M., Marscher, A. P., Ibánez, J. M., \& Alberdi, A. 1997, ApJL, 482, L33

Gurwell, M. A., Peck, A. B., Hostler, S. R., Darrah, M. R., \& Katz, C. A. 2007, in ASP Conf. Ser. 375, From Z-Machines to ALMA: (Sub)millimeter Spectroscopy of Galaxies, ed. A. J. Baker et al. (San Francisco, CA: ASP), 234

Hagen-Thorn, V. A. 1997, AstL, 23, 19

Hagen-Thorn, V. A., Efimova, N. V., Larionov, V. M., et al. 2009, ARep, 53,510

Hill, J. E., Burrows, D. N., Nousek, J. A., et al. 2004, Proc. SPIE, 5165, 217

Homan, D. C., Kadler, M., Kellermann, K. I., et al. 2009, ApJ, 706, 1253

Hughes, P. A., Aller, H. D., \& Aller, M. F. 1985, ApJ, 298, 301

Hughes, P. A., Aller, H. D., \& Aller, M. F. 1989, ApJ, 341, 68

Jorstad, S. G., Marscher, A. P., Larionov, V. M., et al. 2010, ApJ, 715, 362

Jorstad, S. G., Marscher, A. P., Lister, M. L., et al. 2005, AJ, 130, 1418

Jorstad, S. G., Marscher, A. P., Mattox, J. R., et al. 2001, ApJS, 134, 181

Jorstad, S. G., Marscher, A. P., Stevens, J. A., et al. 2007, AJ, 134, 799

Kaspi, S., Smith, P. S., Netzer, H., et al. 2000, ApJ, 533, 631

Kassis, M., Adams, J. D., Hora, J. L., Deutsch, L. K., \& Tollestrup, E. V. 2008, PASP, 120, 874

Kellermann, K. I., Lister, M. L., Homan, D. C., et al. 2004, ApJ, 609, 539

Komissarov, S. S., \& Falle, A. E. G. 1997, MNRAS, 288, 833

Kovalev, Y. Y., Aller, H. D., Aller, M. F., et al. 2009, ApJL, 696, L17

Laing, R. A. 1980, MNRAS, 193, 439

Larionov, V. M., Jorstad, S. G., Marscher, A. P., et al. 2008, A\&A, 492, 389

Larsson, S. 2013, in 2012 Fermi Symposium Proceedings, ed. N. Omodel, T. Brandt, \& C. Wilson-Hodge, eConf C121028

León-Tavares, J., Chavushyan, V., Patiño-Álvarez, V., et al. 2013, ApJL, 763, L36

Lister, M. L., Aller, M., Aller, H., et al. 2011, ApJ, 742, 27

Lister, M. L., Cohen, M. H., Homan, D. C., et al. 2009, AJ, 138, 1874

Lyutikov, M., Pariev, V. I., \& Gabuzda, D. C. 2005, MNRAS, 362, 1078

Malmrose, M. P., Marscher, A. P., Jorstad, S. G., Nikutta, R., \& Elitzur, M. 2011, ApJ, 732, 116

Marscher, A. P. 2006, in AIP Conf. Proc. 856, Relativistic Jets: The Common Physics of AGN, Microquasars, and Gamma-Ray Bursts, ed. P. A. Hughes \& J. N. Bregman (Melville, NY: AIP), 1

Marscher, A. P. 2012, in 2011 Fermi Symposium, eConf C110509 (arXiv:1201.5402)

Marscher, A. P. 2013, in 2012 Fermi Symposium, eConf C121028 (arXiv:1304.2064)

Marscher, A. P., \& Gear, W. K. 1985, ApJ, 298, 114

Marscher, A. P., Gear, W. K., \& Travis, J. P. 1992, in Variability of Blazars, ed. E. Valtaoja \& M. Valtonen (Cambridge: Cambridge Univ. Press), 85
Marscher, A. P., \& Jorstad, S. G. 2010, in Fermi Meets Jansky-AGN at Radio and Gamma-Rays, ed. T. Savolainen, E. Ros, R. W. Porcas, \& J. A. Zensus (Bonn: Max-Planck-Institut für Radioastronomie), 171

Marscher, A. P., Jorstad, S. G., D’Arcangelo, F. D., et al. 2008, Natur, 452, 966

Marscher, A. P., Jorstad, S. G., Larionov, V. M., et al. 2010, ApJL, 710, L126

Max-Moerbeck, W., Richards, J. L., Pavlidou, V., et al. 2010, in Fermi Meets Jansky-AGN at Radio and Gamma-rays, ed. T. Savolainen et al. (Bonn: Max-Planck-Institute fur Radioastronomie), 77

McKinney, J. C. 2006, MNRAS, 367, 1797

Mead, A. R. G., Ballard, K. R., Brand, P. W. J. L., et al. 1990, A\&AS, 83, 183

Meier, D. L., Koide, S., \& Uchida, Y. 2000, Sci, 291, 84

Nalewajko, K., \& Begelman, M. C. 2012, MNRAS, 427, 2480

Narayan, R., \& Piran, T. 2012, MNRAS, 420, 604

Narayan, R., \& Tchekhovskoy, A. 2009, ApJ, 697, 1681

Natali, F., Giallongo, E., Cristiani, S., \& La Franca, F. 1998, AJ, 115, 397

Nolan, P. L., Abdo, A. A., Ackermann, M., et al. 2012, ApJS, 199, 31

Pacciani, L., Vittorini, V., Tavani, M., et al. 2010, ApJL, 716, L170

Poole, T. S., Breeveld, A. A., Page, M. J., et al. 2008, MNRAS, 383, 627

Pushkarev, A. B., Hovatta, T., Kovalev, Y. Y., et al. 2012, A\&A, 545, 113

Raiteri, C., Villata, M., Chen, W. P., et al. 2008, A\&A, 485, L17

Raiteri, C. M., Villata, M., Aller, M. F., et al. 2011, A\&A, 534, 87

Raiteri, C. M., Villata, M., Larionov, V. M., et al. 2007, A\&A, 473, 819

Raiteri, C. M., Villata, M., Smith, P. S., et al. 2012, A\&A, 545, 48

Sasada, M., Uemura, M., Fukazawa, Y., et al. 2012, PASJ, 64, 58

Savolainen, T., Wiik, K., Valtaoja, E., Jorstad, S. G., \& Marscher, A. P. 2002, A\&A, 394, 851

Schleger, D. J., Finkbeiner, D. P., \& Davis, M. 1998, ApJ, 500, 525

Schmidt, G. D., Stockman, H. S., \& Smith, P. S. 1992, ApJL, 398, L57

Shepherd, M. C. 1997, in ASP Conf. Ser. 125, Astronomical Data Analysis Software and Systems VI, ed. G. Hunt \& H. E. Payne (San Francisco, CA: ASP), 77

Sikora, M., Stawarz, L., Moderski, R., Nalewajko, K., \& Madejski, G. M. 2009, ApJ, 704, 38

Smith, P. S., Elston, R., Berriman, G., Allen, R. G., \& Balonek, T. J. 1988, ApJL, 326, L39

Smith, P. S., Montiel, E., Rightley, S., Turner, J., \& Schmidt, G. D. 2009, in 2009 Fermi Symposium Proceedings, eConf C091122 (arXiv:0912.3621)

Smith, P. S., Schmidt, G. D., \& Jannuzi, B. T. 2011, in 2011 Fermi Symposium Proceedings, eConf C110509 (arXiv:1110.6040)

Sokolov, A. S., Marscher, A. P., \& McHardy, I. M. 2004, ApJ, 613, 725

Tchekhovskoy, A., McKinney, J. C., \& Narayan, R. 2008, MNRAS, 388, 551

Teräsranta, H., Tornikoski, M., Mujunen, A., et al. 1998, A\&AS, 132, 305

Timmer, J., \& Koenig, M. 1995, A\&A, 300, 707

Vanden Berk, D. E., Richards, G. T., Bauer, A., et al. 2001, AJ, 122, 549

Vercellone, S., D’Ammando, F., Vittorini, V., et al. 2010, ApJ, 712, 405

Vercellone, S., Striani, E., Vittorini, V., et al. 2011, ApJL, 736, L38

Villata, M., Raiteri, C. M., Aller, M. F., et al. 2007, A\&A, 464, L5

Villata, M., Raiteri, C. M., Balonek, T. J., et al. 2006, A\&A, 453, 817

Vlahakis, N., \& Königl, A. 2004, ApJ, 605, 656

Wardle, J. F. C., \& Kronberg, P. P. 1974, ApJ, 194, 249

Wehrle, A. E., Marscher, A. P., Jorstad, S. G., et al. 2012, ApJ, 758, 72 